\title{
Cutting and gluing with running couplings in $\mathcal{N}=2$ QCD
}

\author{
Johannes Aspman $\odot,{ }^{*}$ Elias Furrer $\odot,^{\dagger}$ and Jan Manschot $\odot^{\ddagger}$ \\ School of Mathematics, Trinity College, Dublin 2, Ireland \\ and Hamilton Mathematical Institute, Trinity College, Dublin 2, Ireland
}

(Received 17 August 2021; accepted 7 October 2021; published 25 January 2022)

\begin{abstract}
We consider the order parameter $u=\left\langle\operatorname{Tr} \phi^{2}\right\rangle$ as a function of the running coupling constant $\tau \in \mathbb{U}$ of asymptotically free $\mathcal{N}=2 \mathrm{QCD}$ with gauge group $S U(2)$ and $N_{f} \leq 3$ massive hypermultiplets. If the domain for $\tau$ is restricted to an appropriate fundamental domain $\mathcal{F}_{N_{f}}$, then the function $u$ is one to one. We demonstrate that these domains consist of six or less images of an $\operatorname{SL}(2, \mathbb{Z})$ keyhole fundamental domain, with appropriate identifications of the boundaries. For special choices of the masses, $u$ does not give rise to branch points and cuts, such that $u$ is a modular function for a congruence subgroup $\Gamma$ of $\operatorname{SL}(2, \mathbb{Z})$ and the fundamental domain is $\Gamma \backslash \mathbb{R}$. For generic masses, however, branch points and cuts are present, and subsets of $\mathcal{F}_{N_{f}}$ are being cut and glued upon varying the mass. We study this mechanism for various phenomena, such as the decoupling of hypermultiplets, the merging of local singularities, as well as the merging of nonlocal singularities which give rise to superconformal Argyres-Douglas theories.
\end{abstract}

DOI: 10.1103/PhysRevD.105.025021

\section{INTRODUCTION}

A manifestation of $S$ duality or strong-weak coupling duality is the equivalent dynamics of a quantum field theory at distinct values of its coupling constant [1-5]. A natural question for such a quantum field theory is the determination of a domain for the coupling constant parametrizing inequivalent quantum field theories. We address this question for asymptotically free $\mathcal{N}=2$ Yang-Mills theories with gauge group $S U(2)$ and $N_{f} \leq 3$ fundamental hypermultiplets. To this end, we consider the order parameter for the Coulomb branch, which is a function of the running coupling $\tau$ invariant under $S$ duality $[3,6-10]$. We put forward a fundamental domain $\mathcal{F}_{N_{f}}$ for $\tau$ such that the function is one to one. Part of our motivation is the $u$-plane integral [11,12], which is a physical approach to Donaldson invariants and other topological gauge-theoretic invariants of smooth compact four-manifolds. This approach involves an integral over the Coulomb branch of the theory. Recently, the change of variables from $u$ to $\tau$ has been instrumental for the evaluation of the integral for generic

\footnotetext{
*aspmanj@maths.tcd.ie

†urrere@maths.tcd.ie

*manschot@maths.tcd.ie
}

Published by the American Physical Society under the terms of the Creative Commons Attribution 4.0 International license. Further distribution of this work must maintain attribution to the author(s) and the published article's title, journal citation, and DOI. Funded by SCOAP ${ }^{3}$. four-manifolds [13-25], which suggests a potential fundamental role for this parametrization of the Coulomb branch.

The Coulomb branches of the rank 1 theories mentioned above are complex one-dimensional, and parametrized by the Higgs vacuum expectation value $u=\frac{1}{16 \pi^{2}}\left\langle\operatorname{Tr} \phi^{2}\right\rangle, \phi$ being the complex scalar of the $\mathcal{N}=2$ vector multiplet [6] (see Refs. [26,27] for a review). In general, these order parameters are functions of the running coupling $\tau$, the masses $m_{i}$ of the hypermultiplets and the dynamical scales $\Lambda_{N_{f}}$ generated by the renormalization group flow.

Before discussing the results of this paper, let us briefly recall relevant aspects of the pure theory, i.e. $N_{f}=0$. The duality group of this theory is isomorphic to $\Gamma^{0}(4)$ and acts on $\tau$ through linear fractional transformations [6]. Its Coulomb branch can be parametrized as the corresponding fundamental domain, $\Gamma^{0}(4) \backslash \mathbb{H}[6,11]$. See Fig. 2. The cusps of $\Gamma^{0}(4) \backslash \mathbb{M}$ correspond to the strong coupling singularities of the theory. Moreover, the order parameter $u$ is a weakly holomorphic modular function for this group.

For the massive theories with $N_{f} \leq 3$, we find a number of new phenomena. To study these theories, we consider their order parameters as roots of certain degree six polynomials constructed from the Seiberg-Witten (SW) curves. These polynomials in turn encode many of the interesting structures of the Coulomb branches. For example, their ramification loci include the Argyres-Douglas (AD) theories, where the curves degenerate, as well as branch points. We show that the fundamental domain of $u$ can be described as six or less copies of the corresponding fundamental domain of the full modular group $\operatorname{SL}(2, \mathbb{Z})$ as 


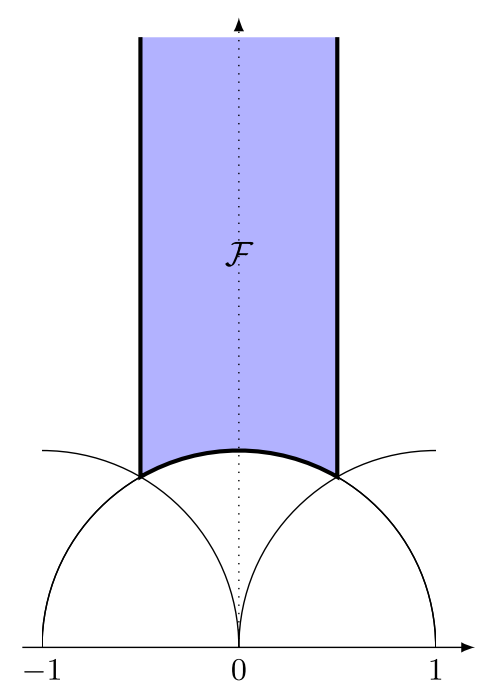

FIG. 1. The keyhole fundamental domain $\mathcal{F}$ of $\operatorname{SL}(2, \mathbb{Z})$. The vertical sides are identified, as well as the two halves of the boundary arc on the unit circle.

displayed in Fig. 1. The cusps of these domains correspond to the singularities of the physical theory and the width of each cusp to the number of hypermultiplets becoming massless there. The images of the $\operatorname{SL}(2, \mathbb{Z})$ fundamental domains under the map $u(\tau)$ provide intriguing partitions of the $u$ plane. See for example Figs. 4, 5, 8 and 13.

Since the polynomials are order six in $u$ it is in general not possible to find the roots and solve for $u$ in terms of the coefficients. Only for special configurations of the masses, e.g. equal masses in $N_{f}=2$ and one nonzero mass in $N_{f}=3$, does the polynomial split over the field of modular functions for a congruence subgroup of $\operatorname{SL}(2, \mathbb{Z})$, and we can thus find explicit closed expressions for $u$ in terms of known modular forms, reproducing and extending previous results [28-32].

For generic choices of the masses, the function $u(\tau)$ gives rise to branch points $\tau_{\mathrm{bp}}$, where $u-u\left(\tau_{\mathrm{bp}}\right) \sim$ $\sqrt{\tau-\tau_{\mathrm{bp}}}+\cdots$ does not return to itself as $\tau$ encircles $\tau_{\mathrm{bp}}$. While the branch points, and the inevitable branch cuts, obstruct the identification of $\mathcal{F}_{N_{f}}$ as a quotient $\Gamma \backslash \mathbb{M}$ with $\Gamma$ a congruence subgroup, they provide a mechanism for $\mathcal{F}_{N_{f}}$ to evolve as function of the mass. More precisely, the branch points move in the domain $\mathcal{F}_{N_{f}}$ upon varying the masses, and the domain $\mathcal{F}_{N_{f}}$ is literally cut and glued along the branch cuts. This provides a way to analyze how the domain evolves as a function of the masses. We have studied these phenomena in detail in the following limits:

(i) Decoupling of a hypermultiplet: A hypermultiplet decouples in the limit that its mass goes to infinity, $m \rightarrow \infty$. We demonstrate that, in this situation, a branch cut disconnects (or cuts) the strong coupling cusp associated to this hypermultiplet from the rest of the domain. At the same time, the sides of the branch cut are identified to the sides of another branch cut. In this way, the strong coupling cusp is glued back to the weakly coupled cusp, near $i \infty$, where these branch points and cuts disappear in the limit $m \rightarrow \infty$. As a result, the periodicity at $i \infty$ increases by 1 in the limit, while the cusp has disappeared from the strongly coupled region. This is displayed for $N_{f}=1$ in Figs. 9 and 10.

(ii) Merging of local singularities: For a generic choice of masses, the theory with $N_{f}$ hypermultiplets has $N_{f}+2$ distinct strong coupling singularities in the $u$ plane, where dyons become massless and the effective field theory breaks down. By tuning the masses to special values, the singularities for $l$ mutually local dyons can merge in the $u$ plane. We demonstrate that such cases give rise to a cusp with width $l>1$ in $\mathcal{F}_{N_{f}}$. Moreover, when perturbing away from such a special value of the masses, we find that two branch cuts develop from the cusp, which disconnect the singularity in $\mathcal{F}_{N_{f}}$. This is displayed for $N_{f}=2$ in Fig. 14.

(iii) Merging of nonlocal singularities (AD theories): The dynamics is quite different if we tune the masses to special values where singularities corresponding to nonlocal dyons collide in the $u$ plane. Such singularities give rise to superconformal $A D$ field theories [33-38]. In such a situation, we find that two branch points in $\mathcal{F}_{N_{f}}$ typically come together and annihilate at the preimage $\tau_{\mathrm{AD}}$ of the $\mathrm{AD}$ singularity $u_{\mathrm{AD}}$. The two branch cuts join at $\tau_{\mathrm{AD}}$ in the interior of $\mathcal{F}_{N_{f}}$, and disconnect a region from $\mathcal{F}_{N_{f}}$ with the "nonlocal" cusps. ${ }^{1}$ Thus $\mathcal{F}_{N_{f}}$ consists then of $<6$ copies of the $\operatorname{SL}(2, \mathbb{Z})$ fundamental domain, and the AD point is in a sense a remnant of the disconnected region. On the other hand if we take the appropriate scaling limit near the conformal field theory point [34], we find that the disconnected region is a fundamental domain for the order parameter of the $\mathrm{AD}$ theory. If no other branch points remain in $\mathcal{F}_{N_{f}}$, then the order parameters become modular functions for a congruence subgroup. This is displayed in Figs. 7, 12 and 17 for $N_{f}=1,2,3$.

Let us briefly return to the $u$ plane integral. The change of variables from $u$ to $\tau$ gives rise to the factor $d u / d \tau$ in the integrand. Interestingly, $d u / d \tau$ can be expressed in the case of the $N_{f}=0$ theory in terms of the discriminant $\Delta$ and $d u / d a$ [11], which is a consequence of a relation between the prepotential and $u[28,39]$. Up to numerical constants, $\Delta$ and $d u / d a$ are precisely the two gravitational couplings of the topological theory $[40,41]$, such that $d u / d \tau$ is

\footnotetext{
${ }^{1}$ If we view $\mathcal{F}_{N_{f}}$ as a sphere with $3+N_{f}$ punctures, then the region is pinched off rather than cut off.
} 
naturally included. Extending previous work on the massless $N_{f} \leq 3$ [31], we derive a further generalization for all cases $N_{f} \leq 4$ with generic masses. We also discuss how this relation encodes interesting information on the special points of the Coulomb branch, specifically the branch points.

We have organized the paper in the following way. In Sec. II, we review the SW solutions for the $N_{f} \leq 3$ theories and then derive a sextic polynomial for $u$, which is crucial for the construction of fundamental domains for the coupling $\tau$. Section II D discusses partitions of the $u$ plane induced by $\mathcal{F}_{N_{f}}$. Section III derives a generalization of Matone's relation for the generic mass cases, as well as giving a discussion on what information it encodes for the Coulomb branch. We then go on to analyze explicit examples in Secs. IV-VI. We conclude with a brief discussion on our findings as well as some possible further directions in Sec. VII. As a by-product of our analyses we also propose an expression for the beta functions of the massive $N_{f} \leq 3$ theories, generalizing the results of $[42,43]$.

\section{FUNDAMENTAL DOMAINS FOR $S U(2)$ SQCD}

In this Section we develop techniques to determine a fundamental domain for the effective coupling of the asymptotically free $\mathcal{N}=2, S U(2)$ supersymmetric quantum chromodynamics (SQCD) theories.

\section{A. The SW solutions}

We recall a few essential aspects of the SW solutions for these theories, which we use to analyze $u$ as function of $\tau$. The gauge group $S U(2)$ is spontaneously broken to $U(1)$ on the Coulomb branch. The order parameter for this branch is the vacuum expectation value $u$, defined as

$$
u=\frac{1}{16 \pi^{2}}\left\langle\operatorname{Tr} \phi^{2}\right\rangle_{\mathbb{R}^{4}} \in \mathcal{B}_{N_{f}},
$$

where the trace is in the two-dimensional representation of $S U(2)$. Topologically, $\mathcal{B}_{N_{f}}$ is the complex plane $\mathbb{C}$ minus $2+N_{f}$ singular points (for generic masses).

The scalar field related to the photon in the low energy effective field theory is $a$, while $a_{D}$ is related to the dual photon. The SW solution identifies these fields as periods of a specific differential $\lambda$ over two dual cycles, $A$ and $B$, of an elliptic curve with complex structure $\tau$,

$$
a=\int_{\gamma} \lambda, \quad a_{D}=\int_{\gamma_{D}} \lambda
$$

To list the SW curves of the theories with $N_{f} \leq 3$ hypermultiplets, let $\Lambda_{N_{f}}$ be the scale of the theory with $N_{f}$ hypermultiplets, and $m_{j}, j=1, \ldots, N_{f}$ be the masses of the hypermultiplets. The SW curves of the theories are given by $[6]^{2}$

$$
\begin{aligned}
N_{f}= & 0: y^{2}=x^{3}-u x^{2}+\frac{1}{4} \Lambda_{0}^{4} x, \\
N_{f}= & 1: y^{2}=x^{2}(x-u)+\frac{1}{4} m \Lambda_{1}^{3} x-\frac{1}{64} \Lambda_{1}^{6}, \\
N_{f}= & 2: y^{2}=\left(x^{2}-\frac{1}{64} \Lambda_{2}^{4}\right)(x-u)+\frac{1}{4} m_{1} m_{2} \Lambda_{2}^{2} x \\
& -\frac{1}{64}\left(m_{1}^{2}+m_{2}^{2}\right) \Lambda_{2}^{4}, \\
N_{f}= & 3: y^{2}=x^{2}(x-u)-\frac{1}{64} \Lambda_{3}^{2}(x-u)^{2} \\
& -\frac{1}{64}\left(m_{1}^{2}+m_{2}^{2}+m_{3}^{2}\right) \Lambda_{3}^{2}(x-u) \\
& +\frac{1}{4} m_{1} m_{2} m_{3} \Lambda_{3} x-\frac{1}{64}\left(m_{1}^{2} m_{2}^{2}+m_{2}^{2} m_{3}^{2}+m_{1}^{2} m_{3}^{2}\right) \Lambda_{3}^{2} .
\end{aligned}
$$

The family of SW curves are Jacobian rational elliptic surfaces with singular fibers [47-50]. Rational in this context means that $g_{2}$ and $g_{3}$ are polynomials in $u$ of degree at most 4 and 6 , respectively [51].

Decoupling a hypermultiplet corresponds to the following double scaling limit [52]

$m_{j} \rightarrow \infty, \quad \Lambda_{N_{f}} \rightarrow 0, \quad m_{j} \Lambda_{N_{f}}^{4-N_{f}}=\Lambda_{N_{f}-1}^{4-\left(N_{f}-1\right)}$.

One can directly decouple more than one hypermultiplet, where the scales of the low energy theories are defined as

$$
\Lambda_{0}^{2}=m \Lambda_{2}, \quad \Lambda_{0}^{4}=m^{3} \Lambda_{3}, \quad \Lambda_{1}^{3}=m^{2} \Lambda_{3},
$$

and $m$ is the equal mass of the hypermultiplets being decoupled. These curves are constructed in such a way that their mathematical discriminants will, up to an overall normalization, correspond to the physical discriminant. This we define as the monic polynomial,

$$
\Delta_{N_{f}}:=\prod_{i=1}^{N_{f}+2}\left(u-u_{i}\right),
$$

with $u_{i}$ being singular points of the effective theory, where hypermultiplets become massless. It is a polynomial of degree $\operatorname{deg} \Delta_{N_{f}}=N_{f}+2$ in $u{ }^{3}$ To see this, we bring the

\footnotetext{
${ }^{2}$ There are other formulations of the SW curve. For example the class $S$ form is $x^{2}=p_{N_{f}}\left(z, u, \Lambda_{N_{f}}, \boldsymbol{m}\right)$ [44-46]. This has the advantage that the $\mathrm{SW}$ differential is canonically determined as $\lambda=x d z$. The analysis in the present paper still holds for these formulations.

${ }^{3}$ One important note is that in [3] another convention is used for the curve of the pure theory. This gives the duality group $\Gamma(2)$ rather than $\Gamma^{0}(4)$ as in the above. The $\Gamma(2)$ convention, however, turns out to not be suitable for the discussion in this paper due to multiplicities of the singularities of the curve.
} 
SW curves (2.3) into Weierstraß form by shifting $x \rightarrow x+\frac{u}{3}+\frac{\Lambda_{3}^{2}}{192} \delta_{3, N_{f}}$, and rescaling $y \rightarrow y / 2$,

$$
\mathcal{W}: y^{2}=4 x^{3}-g_{2} x-g_{3},
$$

where $g_{2}=g_{2}\left(u, \boldsymbol{m}, \Lambda_{N_{f}}\right)$ and $g_{3}=g_{3}\left(u, \boldsymbol{m}, \Lambda_{N_{f}}\right)$ are polynomials in $u, \boldsymbol{m}=\left(m_{1}, \ldots, m_{N_{f}}\right)$ and the scale $\Lambda_{N_{f}}$. The discriminant $\Delta_{N_{f}}$ is unchanged for this change of variables, and equals

$$
\Delta_{N_{f}}=(-1)^{N_{f}} \Lambda_{N_{f}}^{2 N_{f}-8}\left(g_{2}^{3}-27 g_{3}^{2}\right),
$$

where the last factor is the "mathematical" discriminant. The functions $g_{2}$ and $g_{3}$ can be combined to an absolute invariant $\mathcal{J}$,

$$
\mathcal{J}=12^{3} \frac{g_{2}^{3}}{g_{2}^{3}-27 g_{3}^{2}} .
$$

As opposed to $g_{2}$ and $g_{3}, \mathcal{J}$ is invariant under admissible changes of variables. Two curves are isomorphic if and only if they have the same absolute invariant $\mathcal{J}$. Since $g_{2}(u, \boldsymbol{m}, \Lambda)$ and $g_{3}(u, \boldsymbol{m}, \Lambda)$ are polynomial functions of $u$, $\boldsymbol{m}$ and $\Lambda$ for the SW curves, $\mathcal{J}$ is naturally a rational function $\mathcal{J}(u, \boldsymbol{m}, \Lambda)$ of these variables. On the other hand, the modular Weierstraß form expresses $\mathcal{J}$ in terms of the complex structure $\tau$, namely as the modular $j$ invariant $j(\tau)$ [see (A18) for a definition].

$$
\mathcal{J}(u, \boldsymbol{m}, \Lambda)=j(\tau) .
$$

This allows us to obtain $u$ as function of $\tau$, which is physically the effective coupling constant. Cusps are points where $j(\tau)=\infty$, which correspond to $\tau \in\{i \infty\} \cup \mathbb{Q}$. The $j$ function has fundamental domain $\mathcal{F}=\operatorname{SL}(2, \mathbb{Z}) \backslash \mathbb{M}$, which is typically taken to be the keyhole fundamental domain displayed in Fig. 1. In other words, the function $j: \mathcal{F} \rightarrow \mathbb{C}$ is a bijective map.

\section{B. Partitioning the upper half-plane}

We are interested in determining the fundamental domains $\mathcal{F}_{N_{f}}$ for the effective coupling $\tau$ for a theory with $0 \leq N_{f}<4$. Let us consider $u$ as a function,

$$
u: \mathbb{H} \rightarrow \mathcal{B}_{N_{f}},
$$

and study the analytic properties of this map. We will discuss later the dependence of $\mathcal{F}_{N_{f}}$ on the masses $\boldsymbol{m}$, which we will make manifest in the notation as $\mathcal{F}_{N_{f}}(\boldsymbol{m})$ or more compactly $\mathcal{F}(\boldsymbol{m})$. We find that for $N_{f} \geq 1$ and generic masses the duality group does not act on $\tau$ by fractional linear transformations. This prevents us from defining a fundamental domain as is customary for a congruence subgroup $\Gamma$ of $\operatorname{SL}(2, \mathbb{Z})$. For any point $\tau \in \mathbb{U}$ there exists a $g \in \Gamma$ such that $g \cdot \tau \in \Gamma \backslash \mathbb{H}$, and no two distinct points $\tau, \tau^{\prime}$ in $\Gamma \backslash \mathbb{W}$ are equivalent to each other under $\Gamma$. Rather, we can compare if points $\tau, \tau^{\prime}$ are equivalent under (2.11): if we define the equivalence relation

$$
\tau \sim \tau^{\prime} \Leftrightarrow u(\tau)=u\left(\tau^{\prime}\right),
$$

then the quotient set $\mathbb{\Psi} / \sim$ is a fundamental domain $\mathcal{F}_{N_{f}}$ for the function $u$. Upon plotting $\mathcal{F}_{N_{f}}$ as a domain in $\mathbb{U}$, we will have to introduce identifications along codimension 1 segments as for $\mathcal{F}$ in Fig. 1.

To determine $\mathcal{F}_{N_{f}}$, we bring (2.9) into a more convenient form. We multiply (2.9) by $\Delta_{N_{f}}$ and bring all terms to one side. This gives the polynomial,

$$
\begin{aligned}
P_{N_{f}}(X) & :=\left(g_{2}(X, \boldsymbol{m}, \Lambda)^{3}-27 g_{3}(X, \boldsymbol{m}, \Lambda)^{2}\right) j-12^{3} g_{2}(X)^{3}, \\
& =a_{6} X^{6}+a_{5} X^{5}+\cdots+a_{1} X+a_{0},
\end{aligned}
$$

where the coefficients $a_{i}=a_{i}(\boldsymbol{m}, \Lambda, j)$ are polynomial functions of $\boldsymbol{m}, \Lambda$ and the $j$ function, $a_{i}(\boldsymbol{m}, \Lambda, j) \in$ $\mathbb{C}[\boldsymbol{m}, \Lambda, j]$. The polynomials (2.13) can thus be viewed as polynomials over the field $\mathbb{C}[\boldsymbol{m}, \Lambda, j]$.

We see that (2.9) is equivalent to $P_{N_{f}}(u)=0$ for $\Delta_{N_{f}} \neq 0$, or in other words, away from the singular locus of the theory. The roots of $P_{N_{f}}$ can therefore be identified with the order parameter of the corresponding SW curve. Recall that we can assign $U(1)_{\mathcal{R}}$ charges $\left[u: m_{i}: x: y\right]=$ $[4: 2: 4: 6]$ to the quantities of the Seiberg-Witten curves [6]. Since $g_{2}$ and $g_{3}$ are polynomials in $u$ by construction, by bringing the SW curves to the Weierstraß form and using that $[u]=4$ we have that the degrees of $g_{2}$ and $g_{3}$ as polynomials in $u$ must be $\operatorname{deg}\left(g_{2}\right)=2$ and $\operatorname{deg}\left(g_{3}\right)=3$. Therefore, $P_{N_{f}}$ is a sextic polynomial in $X$.

For generic masses $\boldsymbol{m}$, the sextic equation $P_{N_{f}}=0$ gives rise to $n=6$ different solutions as functions of $j$, while for special choices of $\boldsymbol{m}$, such as those giving rise to superconformal (AD) theories, we have $2 \leq n \leq 4$ different $j$-dependent solutions and $6-n j$-independent solutions. Since $j: \mathcal{F} \rightarrow \mathbb{C}$ is an isomorphism, the $n \leq 6$ solutions provide a multivalued ( $n$-valued) function over $\mathcal{F}$.

To obtain $u$ as a single-valued function of the effective coupling, we choose a different copy of $\mathcal{F}$ for each of the $n \leq 6$ branches, and appropriately identify the boundaries of these domains. These are related to $\mathcal{F}$ by an element of $\operatorname{SL}(2, \mathbb{Z})$, and their union is

$$
\mathcal{F}_{N_{f}}=\bigcup_{j=1}^{n} \alpha_{j} \mathcal{F}
$$

with $\alpha_{j} \in \mathrm{SL}(2, \mathbb{Z})$. A priori, there is no canonical choice for the $\alpha_{j}$, they are determined up to the action of the duality group of the theory. However, some choices are more natural than others. If we demand that $\mathcal{F}_{N_{f}}$ is 
connected and take $\alpha_{1}=\mathbb{1} \in \mathrm{SL}(2, \mathbb{Z})$, then there is only a finite number of choices for $\mathcal{F}_{N_{f}}$. In some cases, $\mathcal{F}_{N_{f}}$ is a modular curve $\Gamma \backslash \mathbb{W}$ for a congruence subgroup $\Gamma \subseteq \operatorname{SL}(2, \mathbb{Z})$. In such cases, $n$ equals the index of $\Gamma$ in $\operatorname{SL}(2, \mathbb{Z})[53]$ (see also Appendix A 2 for the corresponding definitions for modular curves). For later use, we define the set of $\alpha_{j}$ as $\mathcal{C}_{N_{f}}=\left\{\alpha_{j}, j=1, \ldots, n\right\}$.

For generic masses, $n=6$ and $\mathcal{F}_{N_{f}}$ has $3+N_{f}$ cusps, corresponding to weak coupling $\tau \rightarrow i \infty$ and the $2+N_{f}$ singularities of the theory. We find the widths of the cusps by expanding $j(\tau)=\mathcal{J}\left(u, \boldsymbol{m}, \Lambda_{N_{f}}\right)$ for $\tau$ near the cusp. For general $N_{f} \in\{0,1,2,3\}$, the cusp at infinity has width $h_{\infty}=4-N_{f}$. This is because $q^{-1} \sim j(\tau)=\mathcal{J} \sim u^{4-N_{f}}$, which implies $u(\tau) \sim q^{-\frac{1}{4-N_{f}}}$ (where $q=e^{2 \pi i \tau}$ ). Thus for large $\tau, u(\tau)$ is invariant under $T^{4-N_{f}}$, where $T: \tau \mapsto \tau+1$. Near any singularity $u_{s}$, it is clear that $q^{-1} \sim \frac{1}{\left(u-u_{s}\right)_{s}}$, where $n_{s}$ is the multiplicity of the singularity. Similarly, near $u_{s}$ one finds $u(\tau)-u_{s} \sim q^{\frac{1}{h_{s}}}$. Locally, the function $u(\tau)$ has period $h_{s}$, giving the width $h_{s}$ of the cusp. The widths of all cusps then add up to 6 ,

$$
h_{\infty}+\sum_{s} h_{s}=6 .
$$

As mentioned above, the equation $P_{N_{f}}=0$ gives six different solutions for $u$. A natural question that then arises is which of these six to use as our $u$. In some sense this is of course arbitrary, all of them correspond to the order parameter $u$ simply expressed in different duality frames. On the other hand, the most natural solution is the one corresponding to the weak coupling duality frame where $|u|$ is large for $\tau \rightarrow i \infty$. Since the width of the cusp at infinity is $4-N_{f}$ we see that there is still some ambiguity in this choice as long as $N_{f}<3$, but for $N_{f}=3$ there is exactly one choice. We show in Sec. VI that this has $u \rightarrow-\infty$ for $\tau \rightarrow i \infty$, and it turns out that this choice can be taken for all $N_{f} \leq 3$ theories, and is preserved under the decoupling of hypermultiplets, we therefore make this choice throughout. Note that this sign differs from the conventional choice in the literature $[6,30,31]$.

Different mass configurations can give different decompositions of 6 . When singularities merge, their cusps are identified under the duality group and their widths add up. Moreover a cusp moves from the real axis to infinity upon decoupling of a matter multiplet.

For special choices of the masses, not all singularities correspond to cusps $i \infty$ or the real line; also singularities in the interior of the upper half-plane can occur. The theories at these points are of superconformal or Argyres-Douglas type, and the widths of all cusps add up to $n$.

Yet another aspect of the parametrization by $\tau$ is that for special values of $\tau$ in the interior of $\mathcal{F}$, otherwise distinct solutions can coincide. These are branch points of the solutions, where the function $u(\tau)$ ceases to be meromorphic in $\tau$. The branch points in $\mathcal{F}_{N_{f}}$ emanate a branch cut. We will discuss these aspects in more detail in Sec. II C.

For generic masses the equation $P_{N_{f}}(X)=0$ furthermore defines a Riemann surface, which is a sixfold ramified covering over the classical modular curve $\operatorname{SL}(2, \mathbb{Z}) \backslash \mathbb{M}[54]$. On this Riemann surface, any root $u$ forms a meromorphic map to the Coulomb branch. It would be interesting to study the topology of these surfaces in more detail. See also [55].

Example: pure $S U(2)$.- To give an example, we can study the well-known pure $S U(2)$ curve, $[6,28,29]$. The absolute invariant reads

$$
\mathcal{J}\left(u, \Lambda_{0}\right)=\frac{64}{\Lambda_{0}^{8}} \frac{\left(3 \Lambda_{0}^{4}-4 u^{2}\right)^{3}}{\Lambda_{0}^{4}-u^{2}},
$$

and the sextic equation for this theory is

$$
P_{0}(X)=\Lambda_{0}^{8}\left(\Lambda_{0}^{4}-X^{2}\right) j(\tau)-64\left(3 \Lambda_{0}^{4}-4 X^{2}\right)^{3} .
$$

As discussed above, this equation has 6 independent solutions, and we pick the one that has $u \rightarrow-\infty$ for $\tau \rightarrow i \infty$. The cusp at infinity has then width $h_{\infty}=4$, while the two strong coupling cusps both have unit width corresponding to the multiplicity of the two singularities. Moreover, since $P_{0}(X)$ is an even function of $X$, we naturally choose the fundamental domain $\mathcal{F}_{0}$ such that it is invariant under a shift by half the width, that is $\tau \rightarrow \tau+2$. In this way, we arrive at the following union of six copies of the $\operatorname{SL}(2, \mathbb{Z})$ fundamental domain,

$$
\mathcal{F}_{0}=\mathcal{F} \cup T \mathcal{F} \cup T^{2} \mathcal{F} \cup T^{3} \mathcal{F} \cup S \mathcal{F} \cup T^{2} S \mathcal{F}
$$

which is displayed in Fig. 2. These copies form a fundamental domain for the congruence subgroup $\Gamma^{0}(4)$,

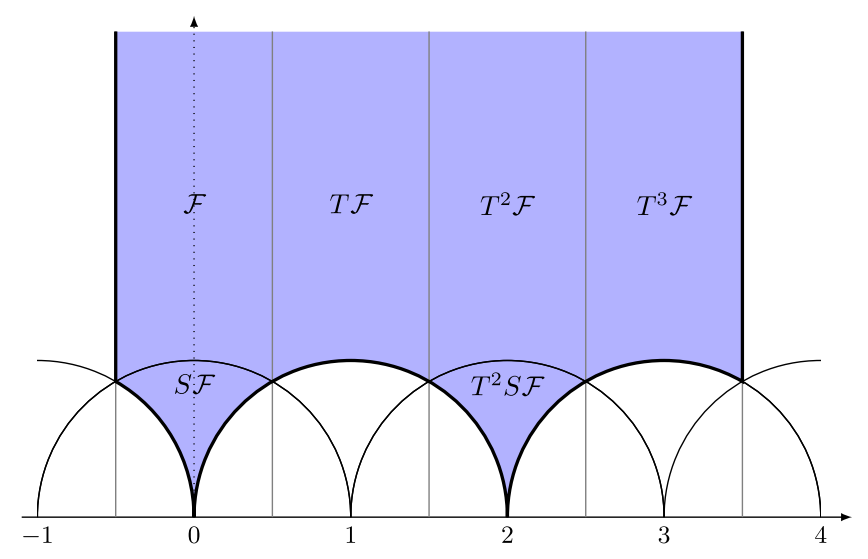

FIG. 2. Fundamental domain of $\Gamma^{0}(4)$. This is the duality group of the pure $S U(2)$ theory. The two cusps on the real line correspond to the strong coupling singularities of the gauge theory, while the cusp at $\tau=i \infty$ corresponds to weak coupling. 
$\mathcal{F}_{0}=\Gamma^{0}(4) \backslash \mathbb{H}$. This demonstrates that the duality group of the theory is $\Gamma^{0}(4)$.

Since the duality group is $\Gamma^{0}(4)$, the order parameter $u$ can be explicitly determined in terms of modular forms for this group. One finds

$$
\begin{aligned}
\frac{u(\tau)}{\Lambda_{0}^{2}} & =-\frac{1}{2} \frac{\vartheta_{2}(\tau)^{4}+\vartheta_{3}(\tau)^{4}}{\vartheta_{2}(\tau)^{2} \vartheta_{3}(\tau)^{2}}=-1-\frac{1}{8}\left(\frac{\eta\left(\frac{\tau}{4}\right)}{\eta(\tau)}\right)^{8}, \\
& =-\frac{1}{8}\left(q^{-1 / 4}+20 q^{1 / 4}-62 q^{3 / 4}+216 q^{5 / 4}+\mathcal{O}\left(q^{7 / 4}\right)\right),
\end{aligned}
$$

with $q=e^{2 \pi i \tau}$. As mentioned above, we have made the (unconventional) choice for the sign of $u$ with $u \rightarrow-\infty$ when $\tau \rightarrow i \infty$.

Even if the duality group is not a congruence subgroup of $\operatorname{SL}(2, \mathbb{Z})$, there is a procedure to find closed expressions for the order parameters in special cases. The sextic equation (2.13) for fixed masses $\boldsymbol{m}$ and scale $\Lambda$ can be viewed as a polynomial over the algebraic field $\mathbb{C}(\Gamma)$ of modular functions on $\Gamma=\operatorname{SL}(2, \mathbb{Z})$. Such nontrivial polynomials define field extensions over $\mathbb{C}(\Gamma)$. By the fundamental theorem of Galois theory, there is a one-to-one correspondence between the Galois group of the field extension and its intermediate fields. Intermediate fields can be obtained by adjoining roots of the polynomial to the base field. Since $P_{N_{f}}(X)$ is a sextic polynomial, for generic masses $\boldsymbol{m}$ it is not possible to find exact expressions for the roots. However, if one of the intermediate fields is known, the polynomial factors over the intermediate field into products of polynomials of lower degree. If the resulting degree is less than or equal to 4, there are closed formulas for the roots.

We find below that in many cases, such as massive $N_{f}=2$ and 3 with one mass parameter, $\mathbb{C}(\Gamma(2))$ for the principal congruence subgroup $\Gamma(2)$ (see Appendix A 1) is an intermediate field. Since the function $\lambda=\frac{\vartheta_{2}^{4}}{\vartheta_{3}^{4}}$ is a Hauptmodul for the genus 0 congruence subgroup $\Gamma(2)$, it is the root of a polynomial of degree $[\Gamma: \Gamma(2)]=6$ over $\mathbb{C}(\Gamma)$. More precisely, there exists a rational function $\mathcal{R}$ with the property that $\mathcal{R}(\lambda(\tau))=j(\tau)$. It is given by

$$
\mathcal{R}(p)=2^{8} \frac{(1+(p-1) p)^{3}}{(p-1)^{2} p^{2}} .
$$

Instead of solving $\mathcal{J}(u, \boldsymbol{m}, \Lambda)=j(\tau)$ we can then rather solve $\mathcal{J}(u, \boldsymbol{m}, \Lambda)=\mathcal{R}(\lambda(\tau))$. If $\mathbb{C}(\Gamma(2))$ is an intermediate field, then the sextic equation corresponding to this equation factors over $\mathbb{C}(\Gamma(2))$. In massive $N_{f}=2,3$ we find that it factors into three quadratic polynomials with coefficients depending on $\lambda$, which can be easily solved analytically. Such rational relations between the $j$ invariant and Hauptmoduln exist for any genus 0 congruence subgroup, which are classified. They allow us to invert the equation $\mathcal{J}(u, \boldsymbol{m}, \Lambda)=j(\tau)$ for a large class of mass parameters, as we demonstrate in the following sections. See also [56-58].

\section{Ramification locus}

The covering $\mathcal{F}_{N_{f}}(\boldsymbol{m}) \rightarrow \mathcal{B}_{N_{f}}$ is not one to one on a discrete subset, namely at points of $\mathcal{F}_{N_{f}}(\boldsymbol{m})$ where the discriminant $D\left(P_{N_{f}}\right)$ vanishes. ${ }^{4}$ In all cases, $N_{f}=0,1,2$, 3 , we find that the discriminant of $P_{N_{f}}$ factorizes as

$$
D\left(P_{N_{f}}\right)=j^{4}(j-1728)^{3}\left(D_{N_{f}}^{\mathrm{AD}}\right)^{3} D_{N_{f}}^{\mathrm{bp}} .
$$

We discuss each of the three factors.

The $\boldsymbol{m}$-independent factor. - The factor $j^{4}(j-1728)^{3}$ is independent of the masses $\boldsymbol{m}$ and can be understood from (2.13). It is immediate that when $j=12^{3}$, every root of $P_{N_{f}}$ has multiplicity at least 2 , and if $j=0$ every root has multiplicity at least 3 . On $\mathbb{t}$ this occurs whenever $\tau \in$ $\operatorname{SL}(2, \mathbb{Z}) \cdot i$ or $\tau \in \operatorname{SL}(2, \mathbb{Z}) \cdot \omega_{3}$, with $\omega_{j}=e^{2 \pi i / j}$. On the modular curve $\operatorname{SL}(2, \mathbb{Z}) \backslash \mathbb{E}$, these orbits collapse to a point and in fact the covering $\pi$ is ramified only over $\{i \infty, i, \alpha\}$, or $j \in\{0,1728, \infty\}$, respectively. This resembles the Belyi functions, which are holomorphic maps from a compact Riemann surface to $\mathbb{P}^{1}(\mathbb{C})$ ramified over precisely these three points $[49,59]$. They can be described combinatorially by so-called dessins d'enfants. Such dessins have also appeared in the context of SW theory [60-62]. For generic masses, the SW family of curves do not satisfy this definition, as there are additional ramification points.

The polynomial $D_{N_{f}}^{\mathrm{AD}}$. - The factor $D_{N_{f}}^{\mathrm{AD}}$ corresponds to AD loci, where two or more singularities coincide $[33,34]$. More precisely, the zero locus of $D_{N_{f}}^{\mathrm{AD}}$ corresponds to the masses for which the Coulomb branch contains AD points. To see this, recall that the AD points correspond to

$$
g_{2}(u, \boldsymbol{m}, \Lambda)=g_{3}(u, \boldsymbol{m}, \Lambda)=0 .
$$

Since $g_{2}$ and $g_{3}$ are polynomials in $u$ of degrees 2 and 3, respectively, we can eliminate $u$ from the above equations and characterize $\mathcal{L}_{N_{f}}^{\mathrm{AD}}$ as the zero locus of a polynomial $D_{N_{f}}^{\mathrm{AD}}$ in $\boldsymbol{m}$,

$$
\mathcal{L}_{N_{f}}^{\mathrm{AD}}=\left\{\boldsymbol{m} \in \mathbb{C}^{N_{f}} \mid D_{N_{f}}^{\mathrm{AD}}(\boldsymbol{m})=0\right\} .
$$

These are precisely the polynomials appearing in Eq. (2.21). From the SW curves we can easily find that they are given by

\footnotetext{
${ }^{4}$ The discriminant of a polynomial $p(X)=X^{n}+a_{n-1} X^{n-1}+$ $\cdots+a_{1} X+a_{0}=\prod_{j=1}^{n}\left(X-r_{j}\right)$ is defined as $D(p)=$ $\prod_{i<j}\left(r_{i}-r_{j}\right)^{2}$, in particular it vanishes if and only if two roots coincide. Since we are interested in finding the zeros of $D(p)$, we are not careful about overall normalization factors.
} 


$$
\begin{aligned}
D_{0}^{\mathrm{AD}}= & 1, \\
D_{1}^{\mathrm{AD}}= & 27 \Lambda_{1}^{3}-64 m^{3}, \\
D_{2}^{\mathrm{AD}}= & \Lambda_{2}^{6}-12 m_{1} m_{2} \Lambda_{2}^{4}+3\left(3 m_{1}^{4}+3 m_{2}^{4}-2 m_{1}^{2} m_{2}^{2}\right) \Lambda_{2}^{2} \\
& -64 m_{1}^{3} m_{2}^{3}, \\
D_{3}^{\mathrm{AD}}= & \Lambda_{3}^{9}-12 \tilde{M}_{2} \Lambda_{3}^{7}+168 \tilde{M}_{3} \Lambda_{3}^{6}-174 \tilde{M}_{4}^{\prime} \Lambda_{3}^{5}+48 \tilde{M}_{4} \Lambda_{3}^{5} \\
& +168 \tilde{M}_{2} \tilde{M}_{3} \Lambda_{3}^{4}-372 \tilde{M}_{3}^{2} \Lambda_{3}^{3}+24 \tilde{M}_{6}^{\prime} \Lambda_{3}^{3}-64 \tilde{M}_{6} \Lambda_{3}^{3} \\
& -24 \tilde{M}_{3} \tilde{M}_{4}^{\prime} \Lambda_{3}^{2}+96 \tilde{M}_{3} \tilde{M}_{4} \Lambda_{3}^{2}+6 \tilde{M}_{2} \tilde{M}_{3}^{2} \Lambda_{3} \\
& -27 \tilde{M}_{8}^{\prime} \Lambda_{3}+8 \tilde{M}_{3}^{3},
\end{aligned}
$$

where for $N_{f}=3$ we have defined the symmetric combinations

$$
\begin{array}{rlrl}
\tilde{M}_{2 k} & =2^{6 k} \sum_{j=1}^{3} m_{j}^{2 k}, & \tilde{M}_{3}=2^{9} \prod_{j=1}^{3} m_{j}, \\
\tilde{M}_{4}^{\prime}=2^{12} \sum_{i<j} m_{i}^{2} m_{j}^{2}, & \tilde{M}_{6}^{\prime}=2^{18} \sum_{i \neq j} m_{i}^{2} m_{j}^{4}, \\
\tilde{M}_{8}^{\prime}=2^{24} \sum_{i<j} m_{i}^{4} m_{j}^{4} . &
\end{array}
$$

The type of singularity that appears for specific masses on these loci are found by studying the order of vanishing of $g_{2}, g_{3}$ and $\Delta$ according to the Kodaira classification,

$$
\begin{aligned}
\text { II }: \operatorname{ord}\left(g_{2}, g_{3}, \Delta\right) & =(1,1,2) \quad \text { or } \quad(2,1,2), \\
\text { III }: \operatorname{ord}\left(g_{2}, g_{3}, \Delta\right) & =(1,2,3), \\
\text { IV }: \operatorname{ord}\left(g_{2}, g_{3}, \Delta\right) & =(2,2,4)
\end{aligned}
$$

See Appendix A 3 for more details. The zero loci of the AD polynomials can be understood as codimension 1 loci in the space $\mathbb{C}^{N_{f}} \ni \boldsymbol{m}$ [34]. For $N_{f}=3$ such a locus is shown in Fig. 3. Argyres-Douglas loci are studied for a more general class of SW theories in [37].

In Sec. II B, we argued that the widths of the different cusps of the $S U(2)$ theories always add up to $n \leq 6$. We will now argue that $n<6$ if and only if $\boldsymbol{m}$ is a zero $\boldsymbol{m}_{\mathrm{AD}}$ of $D_{N_{f}}^{\mathrm{AD}}$. It is possible that some zero of $\Delta$ is also a zero of $g_{2}$. Then the index is given by the degree of the numerator of $j$, which can be smaller than 6. In Secs. IV B, VD, and VID-VIF we study a few examples of $\mathrm{AD}$ theories appearing in the $N_{f}=1,2,3$ theories, and demonstrate that the curve degenerates to Kodaira types II, III or IV. Each singularity type is not exclusive to a specific number of flavors, but appears on the discriminant divisor of the higher $N_{f}$ theories as well [34]. See Secs. VIE and VIF for two explicit examples of this. The three types of $\mathrm{AD}$ theories correspond to 2, 3 or 4 mutually nonlocal states becoming massless at the $\mathrm{AD}$ point. The cusps corresponding to the nonlocal states are disconnected from the rest of the domain, and the branch points collide at an elliptic point

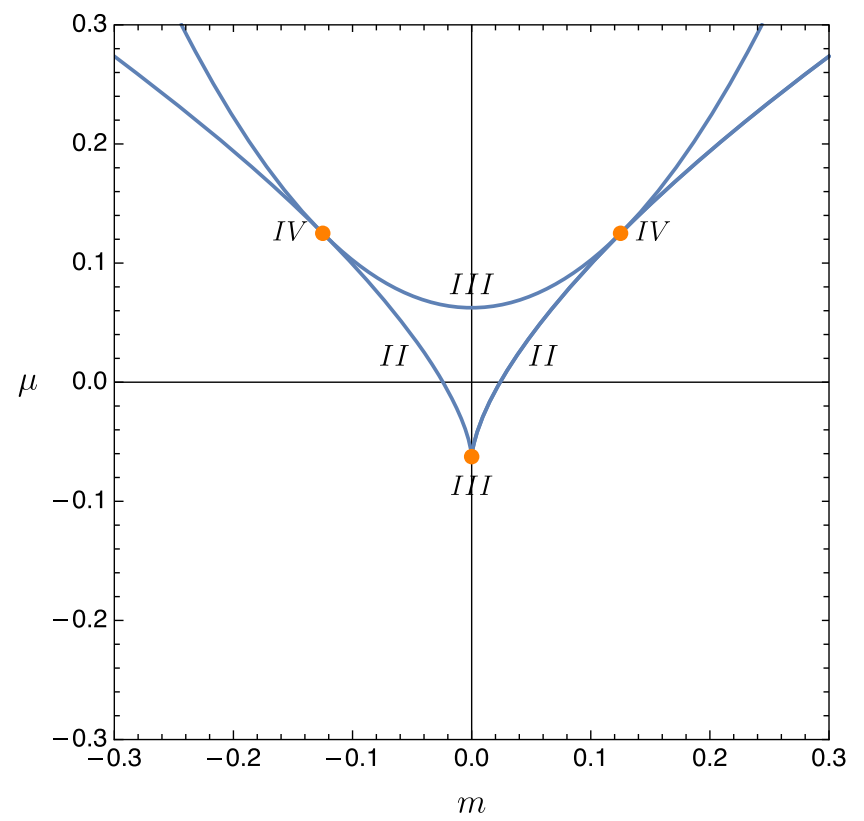

FIG. 3. The $\mathrm{AD}$ locus $\mathcal{L}_{3}^{\mathrm{AD}}$ for $N_{f}=3$ with masses $\boldsymbol{m}=$ $(m, m, \mu)$ in the real $(m, \mu)$ plane, with units $\Lambda_{3}=1$. It is a union of three smooth lines, two of them generically describing type II AD points and the third one type III. The two II lines touch at a III point, while both II lines touch the III line in a type IV AD point.

of the duality group. As a result, the index is reduced by ord $\Delta$, which equals the number of mutually nonlocal states becoming massless, i.e. 2, 3 and 4 for the theories II, III and IV, respectively. Note that the order of vanishing of the discriminant may be larger than zero for ordinary singularities as well, so it is not enough to simply subtract ord $\Delta$ from six to get the index right but rather we should subtract the number of mutually nonlocal states becoming massless at each cusp,

$$
n=6-\#(\text { mutually nonlocal massless dyons). }
$$

This is because for the index to reduce it is necessary for $g_{2}$ and $\Delta$ to have a common root, such that due to (2.8) it is also a root of $g_{3}$ and because of (2.22) therefore an AD point. In the limit $\boldsymbol{m} \rightarrow \boldsymbol{m}_{\mathrm{AD}}$, the $6-n$ copies of $\mathcal{F}_{N_{f}}(\boldsymbol{m})$ corresponding to the regular singularities are removed from the fundamental domain. We have also found mass configurations whose corresponding Coulomb branch contains two (type II) AD points. The correspondence (2.27) nevertheless holds, for a similar argument as presented above.

The polynomial $D_{N_{f}}^{\mathrm{bp}}$. - The last factor $D_{N_{f}}^{\mathrm{bp}}$ corresponds to branch points. These are values of $j$ for which two solutions of $P_{N_{f}}(X)=0$ coincide, such that the map $u: \mathcal{F}_{N_{f}}(\boldsymbol{m}) \rightarrow \mathcal{B}_{N_{f}}$ is not one to one on these points. The identifications are different from the multiple images 
of $\mathcal{F}$ in $\mathcal{B}_{N_{f}}$, which identify the images of the boundary of $\mathcal{F}, \alpha_{j}(\partial \mathcal{F})$, in $\mathcal{F}_{N_{f}}(\boldsymbol{m})$.

The $D_{N_{f}}^{\text {bp }}$ are explicitly given by

$$
\begin{aligned}
D_{0}^{\mathrm{bp}}= & 1, \\
D_{1}^{\mathrm{bp}}= & 27 j \Lambda_{1}^{6}-27 \cdot 2^{14} m^{3} \Lambda_{1}^{3}+2^{20} m^{6}, \\
D_{2}^{\mathrm{bp}}= & \left(m_{1}^{2}-m_{2}^{2}\right)^{2} j^{2} \Lambda_{2}^{8}-128 \Lambda_{2}^{4}\left(216\left(m_{1}^{8}+m_{2}^{8}\right)\right. \\
& -288 m_{1}^{2} m_{2}^{2}\left(m_{1}^{4}+m_{2}^{4}\right)+16 m_{1}^{4} m_{2}^{4}+240 m_{1}^{3} m_{2}^{3} \Lambda_{2}^{2} \\
& -72 m_{1} m_{2}\left(m_{1}^{4}+m_{2}^{4}\right) \Lambda_{2}^{2}+9\left(m_{1}^{4}+m_{2}^{4}\right) \Lambda_{2}^{4} \\
& \left.-42 m_{1}^{2} m_{2}^{2} \Lambda_{2}^{4}-2 m_{1} m_{2} \Lambda_{2}^{6}\right) j \\
& +2^{12}\left(16 m_{1} m_{2}-\Lambda_{2}^{2}\right)^{3} P_{2}^{\mathrm{AD}},
\end{aligned}
$$

and we define $\mathcal{L}_{N_{f}}^{\mathrm{bp}}$ as the zero locus of $D_{N_{f}}^{\mathrm{bp}}$. The expression for $D_{3}^{\mathrm{bp}}$ for generic masses is very long so we do not write it out here, but we can note that it is has degree three in $j$. For later reference we write it out for two special mass configurations

$$
\begin{aligned}
D_{3}^{\mathrm{bp}}(m, m, m)= & 432 m^{4} \Lambda_{3}^{2} j+\left(8 m-\Lambda_{3}\right)^{2}\left(16 m+\Lambda_{3}\right)^{3} \\
& \times\left(64 m+\Lambda_{3}\right), \\
D_{3}^{\mathrm{bp}}(m, 0,0)= & 16 m^{4} \Lambda_{3}^{2} j+\left(8 m-\Lambda_{3}\right)^{3}\left(8 m+\Lambda_{3}\right)^{3} .
\end{aligned}
$$

To show that the zero locus of these polynomials really correspond to branch points we will need some specific details of the corresponding theory and we therefore hold off on this discussion until the respective sections below. We can, however, note that by solving $D_{N_{f}}^{\mathrm{bp}}=0$ for $j$ and plugging it into (2.13) we get the corresponding solutions for $u$. For example, in $N_{f}=1$ we find $u=\frac{4}{3} m^{2}$ and as we will see, away from $m=m_{\mathrm{AD}}=\frac{3}{4} \Lambda_{1}$, this is not part of the discriminant of the curve and therefore does not correspond to a physical singularity of the theory. We denote a branch point of $u$ in $\mathcal{F}_{N_{f}}$ by $\tau_{\mathrm{bp}}$, and its image in $\mathcal{B}_{N_{f}}$ as $u_{\mathrm{bp}}$. As explained in Sec. III C, for generic masses there are two branch points $\tau_{\mathrm{bp}}$ and $\tau_{\mathrm{bp}}^{\prime}$ with image $u_{\mathrm{bp}}=u\left(\tau_{\mathrm{bp}}\right)=$ $u\left(\tau_{\mathrm{bp}}^{\prime}\right)$. Since their image in $\mathcal{B}_{N_{f}}$ is the same, the points $\tau_{\text {bp }}$ and $\tau_{\text {bp }}^{\prime}$ are identified in $\mathcal{F}_{N_{f}}$, even though they appear as distinct points in plots of $\mathcal{F}_{N_{f}}$ in $\mathbb{H}$. A branch cut emanates from each branch point; there can be a single cut connecting both branch points, or two separate cuts which go to either $i \infty$ or to the real axis.

The genus of $\mathcal{F}_{N_{f}}(\boldsymbol{m})$.- - For special choices of the masses $\boldsymbol{m}, \mathcal{F}(\boldsymbol{m})$ coincides with the modular curve $X(\Gamma)$ for a congruence subgroup $\Gamma \in \operatorname{SL}(2, \mathbb{Z})$. Then the genus of $\mathcal{F}(\boldsymbol{m})$ is given by that of $X(\Gamma)$, for which there is the Eq. (A28) in terms of the index $n$, the number of elliptic points $\varepsilon_{2}$ and $\varepsilon_{3}$ and the number of cusps $\varepsilon_{\infty}$. In all examples of such masses $\boldsymbol{m}$ discussed below, we find that
$\mathcal{F}(\boldsymbol{m})$ is a genus zero Riemann surface. In the presence of branch points in $\mathcal{F}(\boldsymbol{m})$, Eq. (A28) needs to be modified. First, we note that, for an $\mathrm{AD}$ theory, $\tau_{\mathrm{AD}}$ corresponds to an elliptic point. In fact, in all AD cases studied here, (2.27) can be expressed as ${ }^{5}$

$$
n=6-2 \varepsilon_{3}-3 \varepsilon_{2},
$$

For $\varepsilon_{\infty}$ there is no simple formula since for example it is not unique in some limit $\boldsymbol{m} \rightarrow \boldsymbol{m}_{\mathrm{AD}}$, but rather depends on the direction in mass space from which $\boldsymbol{m}_{\mathrm{AD}}$ is approached. As the map $u: \mathcal{F}(\boldsymbol{m}) \rightarrow \mathcal{B}_{N_{f}}$ is between Riemann surfaces $\mathcal{F}(\boldsymbol{m})$ and $\mathcal{B}_{N_{f}}$, we can consider the Riemann-Hurwitz formula (A27), which relates their genera $g$. The inverse map $\tau: \mathcal{B}_{N_{f}} \rightarrow \mathcal{F}(\boldsymbol{m})$ can be defined through $\tau=\frac{d a_{D}}{d a}=$ $\frac{d a_{D}}{d u} / \frac{d a}{d u}$, with the periods $a, a_{D}$ given by (2.2). The dependence of $\tau$ on $u$ is holomorphic everywhere $[63,64]$. Then (A27) for the inverse map implies that $0=g_{\mathcal{B}_{N_{f}}} \geq g_{\mathcal{F}(\boldsymbol{m})}$, such that

$$
g_{\mathcal{F}(\boldsymbol{m})}=0 .
$$

Applying this to the Riemann-Hurwitz formula for the ramified covering $\mathcal{F}(\boldsymbol{m}) \rightarrow \mathcal{F}$, we find the number of distinct branch points on $\mathcal{F}(\boldsymbol{m})$ for arbitrary $\boldsymbol{m}$ as

$$
\sum_{\tau_{\mathrm{bp}} \in \mathcal{F}(\boldsymbol{m})}\left(e_{\tau_{\mathrm{bp}}}-1\right)=\varepsilon_{\infty}-3+\varepsilon_{2}+\varepsilon_{3} .
$$

This shows that $\mathcal{F}(\boldsymbol{m})$ is a Riemann sphere with $\varepsilon_{\infty}$ cusps, $\varepsilon_{2}, \varepsilon_{3}$ elliptic points of periods 2 and 3 and $\varepsilon_{\infty}-3+\varepsilon_{2}+$ $\varepsilon_{3}$ branch points. As an example, in massless $N_{f}=1$ (see Sec. IVA) we have $\epsilon_{\infty}=1+3=4$, while all singularities are on $\mathbb{Q}$ and thus $\varepsilon_{2}=\varepsilon_{3}=0$. There is one branch point in $\mathcal{F}(0)$, which agrees with (2.32).

\section{Partitioning the $u$ plane}

An approach to better understand the $u$-plane geometry is to study the partitions that the map $u: \mathcal{F}_{N_{f}} \rightarrow \mathcal{B}_{N_{f}}$ produces on the $u$-plane $\mathcal{B}_{N_{f}}$. Let us study the union (2.14). Now since $u\left(\mathcal{F}_{N_{f}}\right)=\mathcal{B}_{N_{f}}$, it is natural to ask what

$$
\mathcal{T}_{\boldsymbol{m}}=u\left(\bigcup_{j=1}^{n} \alpha_{j} \partial \mathcal{F}\right) \subseteq \mathcal{B}_{N_{f}}
$$

describes. The insight is that while $j: \mathcal{F} \rightarrow \mathbb{C}$ is an isomorphism, it subjects the boundary onto a half-line, ${ }^{6}$

\footnotetext{
${ }^{5}$ The type IV AD point can be viewed as a collision of two elliptic fixed points of period 3 .

${ }^{6}$ This is easy to prove. On the half-lines $i\left[\frac{\sqrt{3}}{2}, \infty\right)$ the $q$ series of $j$ is an alternating series with the same Fourier coefficients as $j$ and therefore real. On the arc $\left\{e^{\varphi i} \mid \varphi \in\left(\frac{\pi}{3}, \frac{2 \pi}{3}\right)\right\}$ the complex conjugate of $j\left(e^{\varphi i}\right)$ is equal to the value of $j$ at the $S$ transform of $e^{\varphi i}$ and therefore equal to $j\left(e^{\varphi i}\right)$.
} 


$$
j(\partial \mathcal{F})=\left(-\infty, 12^{3}\right] \subseteq \mathbb{R} \subseteq \mathbb{C} .
$$

The only other regions in $\mathcal{F}$ where $j$ is real are the $\operatorname{SL}(2, \mathbb{Z})$ images of the half-line $i[1, \infty)$ on the imaginary axis. We can directly apply this to the SW curves, whose $j$ invariant $\mathcal{J}(u, \boldsymbol{m}, \Lambda)$ is identified with $j(\tau)$. The partitioning is then

$$
\mathcal{T}_{\boldsymbol{m}}=\left\{u \in \mathcal{B}_{N_{f}} \mid \mathcal{J}\left(u, \boldsymbol{m}, \Lambda_{N_{f}}\right) \in\left(-\infty, 12^{3}\right]\right\} .
$$

It is included in the level set $\operatorname{Im} \mathcal{J}=0$. Let us therefore study the curves

$$
\operatorname{Im} \mathcal{J}\left(u, \boldsymbol{m}, \Lambda_{N_{f}}\right)=0
$$

which contrary to (2.35) are algebraic curves. It turns out that some of the components of this equation do not belong to the partitioning (2.35), and it is clear that they correspond to components of curves with $j>12^{3}$. Due to the imaginary part, it is instructive to choose coordinates $u / \Lambda_{N_{f}}^{2}=x+i y$. Equation (2.36) is straightforward to compute in terms of zero loci of polynomials in $x$ and $y$. For fixed $\boldsymbol{m}$, they define algebraic varieties

$$
T_{\boldsymbol{m}}(x, y)=0 .
$$

More specifically, they are an $N_{f}$-parameter family of affine algebraic plane curves. For the pure $N_{f}=0$ theory, one finds

$$
\begin{aligned}
T_{0}= & x y\left(81-288 x^{2}+336 x^{4}-128 x^{6}+288 y^{2}-352 x^{2} y^{2}\right. \\
& \left.-128 x^{4} y^{2}+336 y^{4}+128 x^{2} y^{4}+128 y^{6}\right) .
\end{aligned}
$$

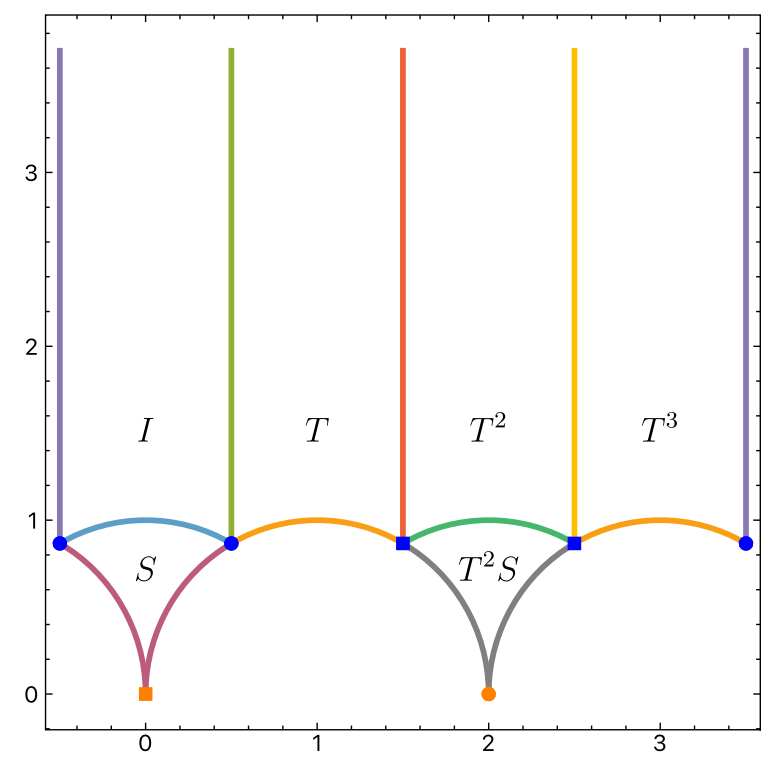

The identification of this partitioning of the $u$ plane for the pure theory is shown in Fig. 4. The defining equations can be computed in full generality for any $N_{f}$, but they are rather lengthy: the polynomials $T_{m}$ for generic masses have total degree $8+N_{f}$. For generic real masses, the polynomials $T_{m}$ have 30,131 and 1081 terms in $N_{f}=1,2$ and 3 , respectively. If we allow the masses to be complex, then we can decompose $m_{i}=\operatorname{Re} m_{i}+i \operatorname{Im} m_{i}$ and the $T_{m}$ are then polynomials in $x, y, \operatorname{Re} m_{i}$, and $\operatorname{Im} m_{i}$. For generic (complex) masses in $N_{f}=1,2$ and 3, $T_{m}$ has 93, 1310 and 48754 terms, respectively.

The polynomials $T_{m}$ are in general reducible. For instance, for $\boldsymbol{m}=(m, m)$ and $\boldsymbol{m}=(m, 0,0), T_{\boldsymbol{m}}$ factors into multiple nontrivial polynomials. It is straightforward to check that $T_{m}$ for given $N_{f}$ flows into $T_{m}$ for $N_{f}-1$ by decoupling one hypermultiplet. This allows us to study the decoupling procedure of the fundamental domains in detail.

The partitioning $\mathcal{T}_{m}$ is a finite union of smooth curves that intersect. The tessellation of $\mathbb{U}$ in $\operatorname{SL}(2, \mathbb{Z})$ images of $\mathcal{F}$,

$$
\mathcal{T}_{\mathbb{H}}=\bigcup_{\alpha \in \operatorname{SL}(2, \mathbb{Z})} \alpha(\partial \mathcal{F})=\left\{\tau \in \mathbb{H} \mid j(\tau) \leq 12^{3}\right\},
$$

has intersection points $\tau \in \operatorname{SL}(2, \mathbb{Z}) \cdot e^{\frac{\pi i}{3}}$, where $j(\tau)=0$. From (2.10) we see that these intersection points correspond to $\mathcal{J}(u, \boldsymbol{m}, \Lambda)=0$, whose only solutions are given by $g_{2}(u, \boldsymbol{m}, \Lambda)=0$ [see Eq. (2.9)]. Since $g_{2}$ is a polynomial in $u$ of degree 2 for all curves (2.3), there are at most two intersection points in $\mathcal{T}_{m}$ corresponding to $\mathcal{J}=0$. As $g_{2}$ is strictly quadratic, there is also always at least one such point. We find below that when the branch points (as

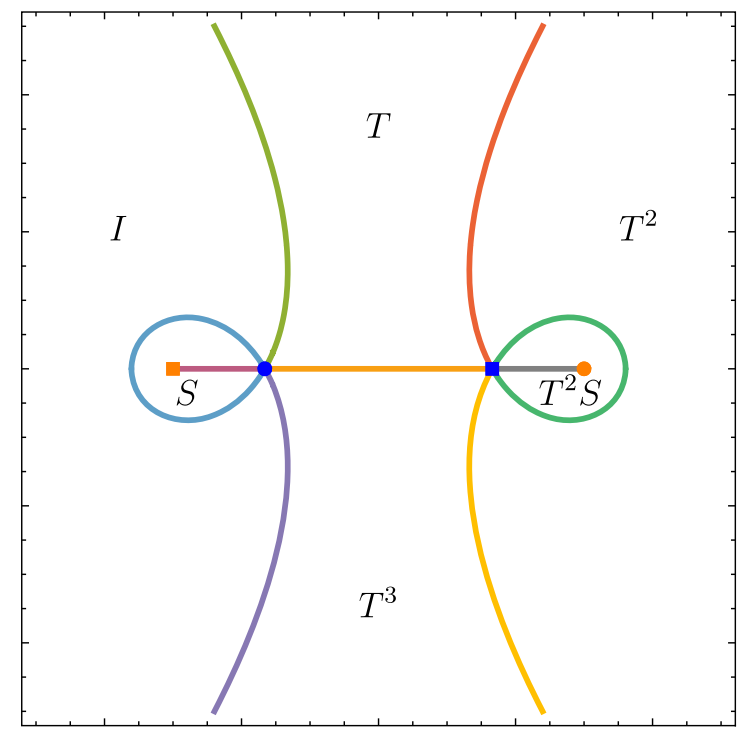

FIG. 4. Identification of the components of the partitioning $\mathcal{T}$ in the pure theory. The $u$-plane $\mathcal{B}_{0}$ is partitioned into six regions $u(\alpha \mathcal{F})$, with the $\alpha \in \operatorname{SL}(2, \mathbb{Z})$ given in both pictures. 
introduced in Sec. II C) belong to $\mathcal{T}_{\boldsymbol{m}}$, they give further intersection points of $\mathcal{T}_{\boldsymbol{m}}$.

One can study how the partitioning is deformed upon varying the masses. For the cases where the branch points belong to $\mathcal{T}_{m}$, the complex $u$ plane is generically partitioned into six regions. When going to the AD locus two or more of these regions shrink to a point together with at least one branch point. At precisely $m=m_{\mathrm{AD}}$, the $u$ plane is then partitioned into $\leq 4$ regions, giving an explanation for the discontinuous decrease in the index in the limit $m \rightarrow m_{\mathrm{AD}}$. This can also be understood directly from the polynomials $T_{\boldsymbol{m}}(x, y)$. For instance, at the point $m=m_{\mathrm{AD}}=\frac{3}{4} \Lambda_{1}$ in $N_{f}=1$, the polynomial $T_{m_{\mathrm{AD}}}(x, y)$ contains a factor $9-24 x+16 x^{2}+16 y^{2}$. Its zero locus in $\mathbb{R}^{2}$ is just a point $x+i y=\frac{3}{4}=u_{\mathrm{AD}} / \Lambda_{1}^{2}$, while the massive deformation away from $m_{\mathrm{AD}}$ describes a curve that encloses a region. For $u_{\text {bp }} \notin \mathcal{T}_{m}$ one needs to cut and glue interior points of different regions and the $u$ plane is therefore partitioned into less than six regions. See for example Fig. 11.

\section{MATONE'S RELATION FOR MASSIVE THEORIES}

In pure $\mathcal{N}=2$ supersymmetric gauge theories, there is a striking expression for the derivative $d u / d \tau$ in terms of the discriminant $\Delta_{0}$ and $d a / d u$. The relation reads [28,39]

$$
\frac{d u}{d \tau}=-4 \pi i \Delta_{0}\left(\frac{d a}{d u}\right)^{2}
$$

Since $u$ is proportional to $\partial F / \partial \Lambda_{0}$, this equation is equivalent to a recursion relation for the prepotential $F$ [65-67]. Moreover, as $\Delta_{0}$ and $d a / d u$ are both topological couplings, this is a useful relation for evaluation of the $u$-plane integral $[11,17,20]$. Similar relations have also been obtained in the massless $N_{f}=1,2,3$ theories [31]. We will refer to a relation of the type (3.1) as Matone's relation.

In this section, we derive a generalization of (3.1) for massive $N_{f}=1,2,3$. Section III A derives expressions for $d a / d u$ and $\Delta_{N_{f}}$ as functions of $\tau$. Section III B derives Matone's relation (3.13) for generic $N_{f} \leq 3$.

\section{A. Periods and Weierstraß form}

We proceed by deriving an expression for $d a / d u$. To this end, recall that $a$ is given as a period integral (2.2), and that the derivative of the SW differential $\lambda$ to $u$ is holomorphic [6]. Therefore, we can express $d a / d u$ in terms of the variables $x$ and $y$ of (2.7)

$$
\frac{d a}{d u}=\frac{\sqrt{2}}{4 \pi} \int_{\gamma} \frac{d x}{y}
$$

where $\gamma$ is one of the cycles of the elliptic curve. To determine this quantity for the theories with $N_{f} \leq 3$, we map the curve $\mathcal{W}$ to the modular Weierstraß form $\tilde{\mathcal{W}}$, $A: \mathcal{W} \rightarrow \tilde{\mathcal{W}}$. See for example [[68], Sec. 7. 1]. The curve $\tilde{\mathcal{W}}$ reads

$$
\tilde{\mathcal{W}}: \tilde{y}^{2}=4 \tilde{x}^{3}-\tilde{g}_{2} \tilde{x}-\tilde{g}_{3},
$$

with the variables related by the map $A$ as

$$
A:\left\{\begin{array}{l}
\tilde{x}=\alpha^{2} x=\wp(z) \\
\tilde{y}=\alpha^{3} y=\wp^{\prime}(z) \\
\tilde{g}_{2}=\alpha^{4} g_{2} \\
\tilde{g}_{3}=\alpha^{6} g_{3}
\end{array},\right.
$$

where $\wp$ is the Weierstra $\beta$ function and $z \in \mathbb{C}$ a coordinate on the curve. Since $\tilde{\mathcal{W}}$ is the modular Weierstraß curve, the variables $\tilde{g}_{2}$ and $\tilde{g}_{3}$ equal

$$
\tilde{g}_{2}=\frac{4 \pi^{4}}{3} E_{4}, \quad \tilde{g}_{3}=\frac{8 \pi^{6}}{27} E_{6}
$$

with $E_{k}$ the Eisenstein series defined in Eq. (A13). We note that the variables for $\mathcal{W}(2.7)$ have weight 0 under modular transformations, while in (3.3) the weights are $\mathrm{wt}\left(\alpha, \tilde{y}, \tilde{x}, \tilde{g}_{2}, \tilde{g}_{3}\right)=(1,3,2,4,6)$. Using the two equations for $\tilde{g}_{2}$ and $\tilde{g}_{3}$, we can solve for $u$ and $\alpha$. The relation

$$
\alpha=\frac{\sqrt{2} \pi}{3} \sqrt{\frac{g_{2}}{g_{3}} \frac{E_{6}}{E_{4}}},
$$

will be particularly useful for us in the next subsection. This relation can also be derived using Picard-Fuchs equations [69].

Now it is straightforward to determine $d a / d u$ (3.2) using the Weierstraß representation of $(\tilde{x}, \tilde{y})$,

$$
\frac{d a}{d u}=\frac{\sqrt{2} \alpha}{4 \pi} \int_{\tilde{\gamma}} \frac{d \tilde{x}}{\tilde{y}}=\frac{\sqrt{2} \alpha}{4 \pi},
$$

where $\tilde{\gamma}$ is the image of the $\gamma$ under the map $A$, with the variable $z$ of $\tilde{x}(z)$ changing from 0 to 1 .

We continue by studying the discriminants of $\mathcal{W}$ and $\tilde{\mathcal{W}}$. Using $E_{4}^{3}-E_{6}^{2}=12^{3} \eta^{24}$ with $\eta$ as in (A6), we find for the discriminant of $\tilde{\mathcal{W}}, \tilde{\Delta}=(2 \pi)^{12} \eta^{24}$. The discriminant of $\mathcal{W}$, $\Delta_{N_{f}}$ (2.8), on the other hand is a polynomial in $u, \boldsymbol{m}$ and $\Lambda$ and therefore has weight 0 . The two discriminants are related by

$$
\tilde{\Delta}=\alpha^{12}(-1)^{N_{f}} \Lambda_{N_{f}}^{8-2 N_{f}} \Delta_{N_{f}},
$$

or substituting $\alpha$ in terms of $d a / d u(3.7)$, 


$$
\eta^{24}=2^{6}(-1)^{N_{f}} \Lambda_{N_{f}}^{2\left(4-N_{f}\right)}\left(\frac{d a}{d u}\right)^{12} \Delta_{N_{f}}
$$

which holds for $0 \leq N_{f} \leq 3$. Similar expressions exist for $N_{f}=4$ and $\mathcal{N}=2^{*}[22,70]$.

Let us consider the case that $\mathcal{W}$ or $\tilde{\mathcal{W}}$ is singular. The curve $\tilde{\mathcal{W}}$ is only singular at the cusps $\tau \in\{i \infty\} \cup \mathbb{Q}$, since $\tilde{\Delta} \sim \eta^{24}$ vanishes at the cusps and is nonvanishing for $\tau$ in the interior of $\llbracket$. From Eq. (3.8) we see that, at the cusps of $\tilde{\mathcal{W}}$ either $d a / d u$ or $\Delta_{N_{f}}$ must vanish. On the other hand, for $\tau$ in the interior of $\mathbb{U}, \tilde{\Delta}$ is nonvanishing. This means that, if $\mathcal{W}$ is singular $\left(\Delta_{N_{f}}=0\right)$ for such values of $\tau, d a / d u$ should diverge. This is exactly what happens at the AD points,

$$
\frac{d u}{d a}\left(\tau_{\mathrm{AD}}\right)=0, \quad \Delta_{N_{f}}\left(u\left(\tau_{\mathrm{AD}}\right)\right)=0, \quad \tau_{\mathrm{AD}} \in \mathbb{H} .
$$

We can further note that $\frac{d u}{d a}(\tau)=0$ is true also for singularities that are cusps and not elliptic points, i.e. $\Delta_{N_{f}}=0$ for $\tau \in \mathbb{Q}$. This is because if $u$ is not an elliptic point, then $g_{2} \neq 0$ and $g_{3} \neq 0$, since otherwise, from $\Delta=g_{2}^{3}-27 g_{3}^{2}$, both would be zero, giving an elliptic point. Then, from Eq. (3.7) we have that $\left(\frac{d u}{d a}\right)^{2}$ is proportional to $\frac{E_{4}}{E_{6}}$. This is a meromorphic modular form of weight -2 for $\operatorname{SL}(2, \mathbb{Z})$, and one can show using modular transformations that it vanishes on $\mathbb{Q}$. Therefore, we have that $\Delta_{N_{f}}=0$ implies $\frac{d u}{d a}=0$.

\section{B. Matone's relation}

We will now give a generalization of Eq. (3.1) that holds also for the massive $N_{f}=1,2,3$ theories. Let us denote by ' the derivative with respect to $u$ keeping $\boldsymbol{m}$ and $\Lambda_{N_{f}}$ fixed. The derivative with respect to $\tau$ is always given explicitly. From the explicit expression for $j$ as function of $\tau$ (A18), it is easy to check that $\frac{d}{d \tau} j=-2 \pi i \frac{E_{6}}{E_{4}} j$. Using the chain rule and (2.10), we can express this as $\frac{d}{d \tau} \mathcal{J}=\mathcal{J}^{\prime} \frac{d u}{d \tau}$. This gives the first important identity,

$$
\frac{d u}{d \tau}=-2 \pi i \frac{E_{6}}{E_{4}} \frac{\mathcal{J}}{\mathcal{J}^{\prime}},
$$

which holds for any SW curve. From Eq. (2.9) we can compute $\mathcal{J}^{\prime}$ in terms of $g_{2}^{\prime}$ and $g_{3}^{\prime}$. Using relations (3.6) and (3.7), we can substitute $E_{6} / E_{4}$ in terms of $g_{2}, g_{3}$ and $d a / d u$. This gives the exact relation

$\frac{d u}{d \tau}=-72 \pi i \frac{g_{3}}{g_{2}} \frac{\mathcal{J}}{\mathcal{J}^{\prime}}\left(\frac{d a}{d u}\right)^{2}=-\frac{8 \pi i}{3} \frac{g_{2}^{3}-27 g_{3}^{2}}{2 g_{2} g_{3}^{\prime}-3 g_{2}^{\prime} g_{3}}\left(\frac{d a}{d u}\right)^{2}$.

An analogous formula for five-dimensional gauge theories was derived from the Picard-Fuchs perspective in [71] [Eq. (4.23)]. Both factors on the rhs are only relative invariants, but their product is an absolute invariant of the curve $\mathcal{W}$. The numerator on the rhs is proportional to the physical discriminant. The equation has modular weight 2 , since both $\frac{d u}{d \tau}$ and $\left(\frac{d a}{d u}\right)^{2}$ are of weight 2 .

For $0 \leq N_{f} \leq 3$, we can compute the corresponding $g_{i}$, and one can rewrite (3.12) as

$$
\frac{d u}{d \tau}=-\frac{16 \pi i}{4-N_{f}} \frac{\Delta_{N_{f}}}{P_{N_{f}}^{\mathrm{M}}}\left(\frac{d a}{d u}\right)^{2}
$$

where we substituted (2.8) for $\Delta_{N_{f}}$, and defined the polynomial $P_{N_{f}}^{\mathrm{M}}$,

$$
P_{N_{f}}^{\mathrm{M}}=\frac{6}{4-N_{f}}(-1)^{N_{f}} \Lambda_{N_{f}}^{2 N_{f}-8}\left(2 g_{2} g_{3}^{\prime}-3 g_{2}^{\prime} g_{3}\right)
$$

The normalization is chosen such that $P_{N_{f}}^{\mathrm{M}}$ is a monic polynomial. Explicit computation gives

$$
\begin{aligned}
P_{0}^{\mathrm{M}}= & 1 \\
P_{1}^{\mathrm{M}}= & u-\frac{4}{3} m_{1}^{2}, \\
P_{2}^{\mathrm{M}}= & u^{2}-\frac{3}{2}\left(m_{1}^{2}+m_{2}^{2}\right) u+2 m_{1}^{2} m_{2}^{2}+\frac{1}{8} m_{1} m_{2} \Lambda_{2}^{2}-\frac{1}{64} \Lambda_{2}^{4} \\
P_{3}^{\mathrm{M}}= & u^{3}-2 M_{2} u^{2}+\left(3 M_{4}^{\prime}+\frac{3}{4} M_{3} \Lambda_{3}-\frac{1}{64} M_{2} \Lambda_{3}^{2}\right) u \\
& +\frac{1}{256} M_{3} \Lambda_{3}^{3}-\frac{1}{4} M_{2} M_{3} \Lambda_{3} \\
& +\frac{1}{32}\left(M_{4}-M_{4}^{\prime}\right) \Lambda_{3}^{2}-4 M_{3}^{2},
\end{aligned}
$$

where we defined

$$
\begin{array}{ll}
M_{2}=m_{1}^{2}+m_{2}^{2}+m_{3}^{2}, & M_{3}=m_{1} m_{2} m_{3}, \\
M_{4}=m_{1}^{4}+m_{2}^{4}+m_{3}^{4}, & M_{4}^{\prime}=\sum_{i<j} m_{i}^{2} m_{j}^{2} .
\end{array}
$$

We note that these polynomials appear in the Picard-Fuchs equations for the periods of these theories and their zeros give regular singular points of the differential equations $[72,73]$. In Appendix B we give an additional proof of the identities (3.13). ${ }^{8}$

\footnotetext{
${ }^{7} \mathrm{We}$ can in fact perform the same computations in the case of $N_{f_{8}}=4$, leading to a similar formula.

${ }^{8}$ The identity (3.13) does in fact not depend on the specific form of the SW curves. Given a Jacobian rational elliptic surface, let $\omega=\int_{\gamma} \frac{d x}{y}$ be the period of the Néron differential on the elliptic curve. Then $\frac{d u}{d \tau}=\frac{1}{3 \pi i} \omega^{2} \Delta /\left(2 g_{2} g_{3}^{\prime}-3 g_{2}^{\prime} g_{3}\right)$, with $u$ a coordinate on $\mathbb{P}^{1}(\mathbb{C})$.
} 


\section{Branch points}

An important difference between $N_{f}=0$ and $N_{f}>0$ are the poles where $P_{N_{f}}^{\mathrm{M}}$ vanishes. To understand these poles as well as zeros of $d u / d \tau$, note that at such points the change of variables between $u$ and $\tau$ is ill defined. We have seen earlier that the change of variables is ill defined at the points where the discriminant $D\left(P_{N_{f}}\right)$ (2.21) vanishes. Indeed if we substitute $\mathcal{J}\left(u, \boldsymbol{m}, \Lambda_{N_{f}}\right)$ for $j(\tau)$ in $D_{N_{f}}^{\mathrm{bp}}, P_{N_{f}}^{\mathrm{M}}$ factors out.

The reason for this is the following. The discriminant of a polynomial $p$ vanishes if and only $p$ has a double root. It can be computed as the resultant of the polynomial and its formal derivative, $D(p) \sim \operatorname{Res}_{X}\left(p, p^{\prime}\right)$ (see also Refs. [35]). ${ }^{9}$ The zero locus $D\left(P_{N_{f}}\right)=0$ of $P_{N_{f}}(X)$ is then given by the solutions to the two equations $P_{N_{f}}(X)=0$ and $P_{N_{f}}{ }^{\prime}(X)=0$. Since $\Delta_{N_{f}} \neq 0$, all solutions can be found by solving the former for $j$ and inserting into the latter. It is straightforward to show that this gives

$$
\frac{g_{2}^{2} g_{3}}{\Delta_{N_{f}}} P_{N_{f}}^{\mathrm{M}}=0
$$

which provides the decomposition (2.21): if $g_{2}=0$ but $g_{3} \neq 0$, then $j=0$. If $g_{3}=0$ but $g_{2} \neq 0$, then $j=12^{3}$. If both $g_{2}=g_{3}=0$, we are in $\mathcal{L}_{N_{f}}^{\mathrm{AD}} \subseteq \mathcal{L}_{N_{f}}^{\Delta}$. Now since the sextic equation is only well defined away from the physical discriminant locus $\mathcal{L}_{N_{f}}^{\Delta}$, where $\Delta_{N_{f}}=0$, the true branch point locus $\mathcal{L}^{\text {bp }}$ is the difference of the Matone locus $\mathcal{L}_{N_{f}}^{\mathrm{M}}=$ $\left\{u \in \mathcal{B}_{N_{f}} \mid P_{N_{f}}^{\mathrm{M}}=0\right\}$ and the discriminant locus,

$$
\mathcal{L}_{N_{f}}^{\mathrm{bp}}=\mathcal{L}_{N_{f}}^{\mathrm{M}} \backslash \mathcal{L}_{N_{f}}^{\Delta}
$$

On the Coulomb branch with $N_{f}$ hypermultiplets there are generically $2+N_{f}$ distinct singular points. For special mass configurations $\boldsymbol{m}$, some singularities can collide. Then $\Delta_{N_{f}}$ has a double root. From above it is clear that this is equivalent to $D\left(\Delta_{N_{f}}\right)=0$, which in turn is equivalent to $\Delta_{N_{f}}=0$ and $\Delta_{N_{f}}^{\prime}=0$. We can again solve the former for $g_{2}$ and $g_{3}$ and insert into the latter to obtain $P_{N_{f}}^{\mathrm{M}} \sim \frac{g_{2}^{\prime}}{g_{3}} \Delta_{N_{f}}=0$. This implies that whenever $\Delta_{N_{f}}$ has a double root, it is also a root of $P_{N_{f}}^{\mathrm{M}}$. It is also observed in all examples below. To be more precise, if $\Delta_{N_{f}}$ contains a root of $d>1$ th order, then $\Delta_{N_{f}}^{\prime}$ has the same root but with multiplicity $d-1$. The excess factors can be extracted by the operation $\operatorname{gcd}\left(\Delta_{N_{f}}, \Delta_{N_{f}}^{\prime}\right)$, where gcd is the polynomial

\footnotetext{
${ }^{9}$ The resultant of two polynomials over a commutative ring is a polynomial of their coefficients which vanishes if and only if the polynomials have a common root. It can be computed as the determinant of their Sylvester matrix.
}

greatest common divisor. The multiple roots are removed from the discriminant by the square-free factorization ${ }^{10}$

$$
\hat{\Delta}_{N_{f}}=\frac{\Delta_{N_{f}}}{\operatorname{gcd}\left(\Delta_{N_{f}}, \Delta_{N_{f}}^{\prime}\right)} .
$$

This reduced discriminant $\hat{\Delta}_{N_{f}}$ has single roots only, concretely we map $\prod_{s}\left(u-u_{s}\right)^{n_{s}}$ to $\prod_{s}\left(u-u_{s}\right)$. This quantity is also of importance for determining gravitational couplings to Seiberg-Witten theory [74]. One can show that $\operatorname{gcd}\left(\Delta_{N_{f}}, \Delta_{N_{f}}^{\prime}\right)$ always divides $P_{N_{f}}^{\mathrm{M}}$, such that

$$
\hat{P}_{N_{f}}^{\mathrm{M}}:=\frac{\hat{\Delta}_{N_{f}}}{\Delta_{N_{f}}} P_{N_{f}}^{\mathrm{M}}
$$

is in fact a polynomial. The branch point Eq. (3.17) is then equivalent to $\hat{P}_{N_{f}}^{\mathrm{M}} / \hat{\Delta}_{N_{f}}=0$, which reduces to

$$
\hat{P}_{N_{f}}^{\mathrm{M}}=0 .
$$

The Matone relation thus always takes the form

$$
\frac{d u}{d \tau}=-\frac{16 \pi i}{4-N_{f}} \frac{\hat{\Delta}_{N_{f}}}{\hat{P}_{N_{f}}^{\mathrm{M}}}\left(\frac{d a}{d u}\right)^{2},
$$

where both $\hat{\Delta}_{N_{f}}$ and $\hat{P}_{N_{f}}^{\mathrm{M}}$ are polynomials. In the subsequent sections we show explicitly that the roots of the denominator (3.21) are precisely the branch points. We note that for generic masses the form (3.22) does not differ from (3.13), because ${ }^{\wedge}$ is trivial when all roots are distinct.

As argued above, $\mathrm{AD}$ points correspond to points $\tau_{\mathrm{AD}}$ in the upper half-plane. Since they lie on the discriminant locus, we exclude them to define the sextic polynomial $P_{N_{f}}$. We will discuss in more detail below that, if the masses approach the AD locus, a branch point in the $u$ plane collides with two mutually nonlocal singularities forming the AD point. The branch point under consideration lifts, while the $N_{f}-1$ other branch points remain for a generic point on the $\mathrm{AD}$ mass locus $\mathcal{L}_{N_{f}}^{\mathrm{AD}}$. Thus for a generic point on the $\mathrm{AD}$ mass locus, $\mathrm{AD}$ points are not branch points of $u(\tau)$. A nongeneric example is the most symmetric AD theory, the IV fiber in $N_{f}=3$, discussed in more detail in Sec. VID. For this theory, $\tau_{\mathrm{AD}}$ corresponds to a singular point of the theory as well as a branch point. As a result, the domain for $\tau$ does not correspond to that of a congruence subgroup of $\operatorname{SL}(2, \mathbb{Z})$.

Since any branch point $\tau_{\mathrm{bp}}$ induces a nontrivial monodromy, $u$ does not have a regular Taylor series at such a point. For instance, if the $u$ plane contains one branch point

\footnotetext{
${ }^{10}$ The polynomial gcd is unique only up to multiplication with invertible constants, we choose it such that $\hat{\Delta}_{N_{f}}$ is again monic.
} 
$u_{\mathrm{bp}}=u\left(\tau_{\mathrm{bp}}\right)$, then we have $u(\tau)-u_{\mathrm{bp}}=\mathcal{O}\left(\sqrt{\tau-\tau_{\mathrm{bp}}}\right)$ as $\tau \rightarrow \tau_{\mathrm{bp}}$. If the leading coefficient is nonzero, then $\frac{d u}{d \tau}$ diverges at $\tau_{\mathrm{bp}}$. Away from the discriminant locus, this can be understood from Eq. (3.22): from Eq. (3.7) we see that $\frac{d a}{d u}$ is regular and nonzero at a branch point, since none of $g_{2}, g_{3}, E_{4}$ and $E_{6}$ diverge or vanish. Thus the zeros of the denominator $\hat{P}_{N_{f}}^{\mathrm{M}}$ correspond to the singular points of $\frac{d u}{d \tau}$, as observed.

This can also be seen directly from the $\mathcal{J}$ invariant of the SW curve. It is easy to show that

$$
\mathcal{J}^{\prime}=36^{3} \frac{g_{2}^{2} g_{3}}{\Delta_{N_{f}}^{2}} P_{N_{f}}^{\mathrm{M}},
$$

which due to Eq. (3.17) vanishes at any branch point $u_{\mathrm{bp}}$. Since for fixed mass and scale $\mathcal{J}(u)$ is rational in $u$, it is a meromorphic function on $\mathcal{B}_{N_{f}}$. Away from the discriminant locus it thus has a Taylor series around $u_{\mathrm{bp}}$, where the linear coefficient is missing. We therefore find

$$
\mathcal{J}(u)-\mathcal{J}\left(u_{\mathrm{bp}}\right)=\mathcal{O}\left(\left(u-u_{\mathrm{bp}}\right)^{n_{\mathrm{bp}}}\right),
$$

with $n_{\mathrm{bp}} \geq 2$. Now we identify $\mathcal{J}(u)=j(\tau)$, which relates the power series of $u$ and $\tau$. For a generic $\tau \in \mathbb{U}, j$ has a regular Taylor series at $\tau$ with nonzero linear coefficient. However if $\tau$ is in the $\operatorname{SL}(2, \mathbb{Z})$ orbit of $i$ or $e^{\frac{\pi i}{3}}, j$ has a zero of order 2 or 3 . Let $n_{\tau_{\text {bp }}} \in\{1,2,3\}$ be this number for a given branch point $\tau_{\mathrm{bp}} \in \mathbb{H}$. Then $\mathcal{J}(u)-\mathcal{J}\left(u_{\mathrm{bp}}\right)=$ $\mathcal{O}\left(\left(\tau-\tau_{\mathrm{bp}}\right)^{n_{\mathrm{bp}_{\mathrm{bp}}}}\right)$, such that from (3.24) we conclude

$$
u(\tau)-u_{\mathrm{bp}}=\mathcal{O}\left(\left(\tau-\tau_{\mathrm{bp}}\right)^{n_{\tau \mathrm{bp}}} / n_{\mathrm{bp}}\right),
$$

where the leading coefficient is strictly nonzero. From this we see that the branch point $\tau_{\mathrm{bp}}$ does not necessarily correspond to an $n_{\mathrm{bp}}$ th root, but since the ratio can cancel $\tau_{\mathrm{bp}}$ it rather corresponds to a branch point of order

$$
\frac{n_{\mathrm{bp}}}{\operatorname{gcd}\left(n_{\mathrm{bp}}, n_{\tau_{\mathrm{bp}}}\right)} \text {. }
$$

It is difficult to compute this integer for a generic branch point, however in all examples discussed below it is equal to 2, which corresponds to a square root.

If the number $\frac{n_{\tau_{\mathrm{bp}}}}{n_{\mathrm{bp}}} \in \mathbb{Q} \backslash \mathbb{Z}$ is larger than 1 , then it is clear that $\frac{d u}{d \tau}\left(\tau_{\mathrm{bp}}\right)=0$. Conversely, if $\frac{n_{\tau_{\mathrm{bp}}}}{n_{\mathrm{bp}}}<1$ then $\frac{d u}{d \tau}\left(\tau_{\mathrm{bp}}\right)=\infty$. We thus see that any branch point has the property that $\frac{d u}{d \tau}$ diverges or vanishes, such that the change of variables from the $u$ plane to the $\tau$ plane is not well defined.

The branch point locus also allows us to find the effective coupling at the AD points. In the limit where the masses approach the $\mathrm{AD}$ locus, $\boldsymbol{m} \rightarrow \boldsymbol{m}_{\mathrm{AD}}$, the $\mathrm{AD}$ point $u_{\mathrm{AD}}$ is the point where branch points $u_{\mathrm{bp}}$ in the $u$ plane merges with mutually nonlocal singularities. While away from $\boldsymbol{m}_{\mathrm{AD}}$ the effective coupling $\tau$ of the singularities remain as distinct cusps on the real line, the branch points move along certain paths inside $\mathbb{H}$. In an AD limit $\boldsymbol{m} \rightarrow \boldsymbol{m}_{\mathrm{AD}}$, a number of pairs of branch points, $\tau_{\mathrm{bp}}$ and $\tau_{\mathrm{bp}}^{\prime}$, coincide at the intersection of copies of $\mathcal{F}$, and the branch cut will then disconnect regions from $\mathcal{F}_{N_{f}}$. The effective coupling of the AD point $\tau_{\mathrm{AD}}$ is therefore given by that of the merged branch points. This is an efficient way to determine $\tau_{\mathrm{AD}}$, which otherwise can only be found by inverting modular functions. Moreover, if the duality group is a congruence subgroup of $\operatorname{SL}(2, \mathbb{Z})$, $\tau_{\mathrm{AD}}$ corresponds to an elliptic point of the duality group.

\section{THE $N_{f}=1$ CURVE}

To make the above discussions more concrete we will now go on to study some specific examples. We will start by including one hypermultiplet. The $N_{f}=1$ theory has been discussed in some detail in Refs. [32,43,75-77].

In the massive $N_{f}=1$ theory, there are three (in general) distinct strong coupling singularities where a hypermultiplet becomes massless. These remain at distinct points in the massless limit, while for special values of the mass two of them can merge into AD points. To analyze the $N_{f}=1$ theory we will start by restricting to the massless case and then go to an $\mathrm{AD}$ mass. Here we can find closed expressions for $u$ in terms of well-known modular forms. Only in the AD case does the theory turn out to be modular. In the end we can use the knowledge gained from these cases to draw some conclusions of the general massive case.

\section{A. The massless theory}

Let us begin with the massless $N_{f}=1$ theory. Using the procedure outlined in Sec. II B we find [29]

$$
\begin{aligned}
\frac{u(\tau)}{\Lambda_{1}^{2}} & =-\frac{3}{2^{\frac{7}{3}}} \frac{\sqrt{E_{4}(\tau)}}{\sqrt[3]{E_{4}(\tau)^{\frac{3}{2}}-E_{6}(\tau)}}, \\
& =-\frac{1}{16}\left(q^{-1 / 3}+104 q^{2 / 3}-7396 q^{5 / 3}+\mathcal{O}\left(q^{8 / 3}\right)\right),
\end{aligned}
$$

where we again have made the choice of solution consistent with our convention, such that $u \rightarrow-\infty$ for $\tau \rightarrow i \infty$. This function also appears as an order parameter in pure $S U(3)$ SW theory [78] as well as in the description of certain elliptically fibered Calabi-Yau spaces [79]. The singularities of the curve are $\frac{u^{3}}{\Lambda_{1}^{6}}=-\frac{3^{3}}{2^{8}}$. They are associated with states of charges $(1,0),(1,1)$ and $(1,2)$ becoming massless. The global $\mathbb{Z}_{3}$ symmetry acts as $T^{-1}: u(\tau-1)=\omega_{3} u(\tau)$, with $\omega_{j}=e^{\frac{2 \pi i}{j}}$. 
By restricting to the imaginary axis, we can perform the $S$ transformation. For this, let $\tau=i \beta$ with $\beta>0$. We have that $E_{4}(i / \beta)=(i \beta)^{4} E_{4}(i \beta)=\beta^{4} E_{4}(i \beta)$. Taking the square root is unambiguous since $E_{4}$ is real on the imaginary axis and $\beta^{4}$ is positive. This gives $E_{4}^{\frac{1}{2}}(i / \beta)=$ $\beta^{2} E_{4}^{\frac{1}{2}}(i \beta)$. On the other hand for $E_{6}$ we have $E_{6}(\tau)=(i \beta)^{6} E_{6}(i \beta)=-\beta^{6} E_{6}(i \beta)$. This implies that the relative sign of $E_{6}$ flips, and it holds for $\tau_{D} \in i \mathbb{R}_{>0}$ that

$$
\begin{aligned}
\frac{u_{D}\left(\tau_{D}\right)}{\Lambda_{1}^{2}} & =-\frac{3}{2^{\frac{7}{3}}} \frac{E_{4}\left(\tau_{D}\right)^{\frac{1}{2}}}{\left(E_{4}\left(\tau_{D}\right)^{\frac{3}{2}}+E_{6}\left(\tau_{D}\right)\right)^{\frac{1}{3}}}, \\
& =\frac{3}{2^{\frac{8}{3}}}\left(1+144 q-3456 q^{2}+596160 q^{3}+\mathcal{O}\left(q^{4}\right)\right) .
\end{aligned}
$$

With the $\mathbb{Z}_{3}$ symmetry $u(\tau-1)=\omega_{3} u(\tau)$ this confirms the strong coupling singularities given above.

The monodromies on the massless $N_{f}=1 u$ plane are [6]

$$
\begin{aligned}
& M_{1}=\left(\begin{array}{cc}
1 & 0 \\
-1 & 1
\end{array}\right)=S T S^{-1}, \\
& M_{2}=\left(\begin{array}{cc}
0 & 1 \\
-1 & 2
\end{array}\right)=(T S) T(T S)^{-1}, \\
& M_{3}=\left(\begin{array}{cc}
-1 & 4 \\
-1 & 3
\end{array}\right)=\left(T^{2} S\right) T\left(T^{2} S\right)^{-1}, \\
& M_{\infty}=\left(\begin{array}{cc}
-1 & 3 \\
0 & -1
\end{array}\right)=P T^{-3},
\end{aligned}
$$

where $P=S^{2}=-1$. Note that these matrices generate the full $\operatorname{SL}(2, \mathbb{Z})$ modular group rather than a (congruence) subgroup. Indeed, as fractional linear transformations acting on the complex structure through their matrix representations, they do not leave $u$ invariant. However, we can consider these matrices as compositions of paths in the fundamental domain, and as such they do leave $u$ invariant. To make the connection to the discussion in Ref. [78] more direct we can note that by using another choice of homology basis in the present case we can construct a different set of monodromies, see for example [52], which exactly coincides with the ones listed for the $S U(3)$ case of [78].

Since $E_{4}$ has a simple zero at $\tau=\omega_{3}$ [and $\operatorname{SL}(2, \mathbb{Z})$ images], $u(\tau)$ has a branch point at $\tau_{\mathrm{bp}}=\omega_{3}$. The function $u(\tau)$ does not possess a Taylor series at $\tau_{\mathrm{bp}}$ and is therefore not holomorphic at $\tau_{\mathrm{bp}}$. Since $u\left(\tau_{\mathrm{bp}}\right)=0$, the branch point of $u(\tau)$ indeed agrees with what is found in Eq. (2.28). Since $u$ is not holomorphic on $\mathbb{H}$, it can also not be classically modular. Another reason why $u$ is not modular is the following. If we define $x:=-16 \frac{u}{\Lambda_{1}^{2}}=$ $q^{-\frac{1}{3}}+\mathcal{O}\left(q^{\frac{2}{3}}\right)$, then one can read off from the curve that $\mathcal{J}=x^{6} /\left(x^{3}-432\right)$. This implies that $u$ should be a
Hauptmodul of an index 6 subgroup of $\operatorname{SL}(2, \mathbb{Z})$ with width $h(\infty)=3$ and width decomposition $6=3+1+1+1$ [see Eq. (A25)]. From the classification of index 6 groups in Table II we see that such a subgroup of $\operatorname{SL}(2, \mathbb{Z})$ does not exist. In fact, no index 6 subgroup of $\operatorname{SL}(2, \mathbb{Z})$ with four cusps exists. This distinguishes massless $N_{f}=1$ from massless $N_{f}=0,2,3$, where the duality groups are congruence subgroups isomorphic to $\Gamma^{0}(4)$ [29].

From Eq. (4.1) one finds

$\frac{d u}{d \tau}=\frac{\pi i \Lambda_{1}^{2}}{2^{\frac{7}{3}}} \frac{E_{4}^{\frac{3}{2}}+E_{6}}{E_{4}^{\frac{1}{2}}\left(E_{4}^{\frac{3}{2}}-E_{6}\right)^{\frac{1}{3}}}, \quad \frac{d a}{d u}=\frac{i\left(E_{4}^{\frac{3}{2}}-E_{6}\right)^{\frac{1}{6}}}{2^{\frac{1}{3}} \sqrt{3} \Lambda_{1}}$.

We can explicitly check that these satisfy Matone's relation, (3.13), for massless $N_{f}=1$,

$$
\frac{d u}{d \tau}=-\frac{16 \pi i}{3} \frac{\Delta}{u}\left(\frac{d a}{d u}\right)^{2} .
$$

The fundamental region

$$
\mathcal{F}_{1}(0)=\bigcup_{\ell=0}^{2} T^{\ell} \mathcal{F} \cup T^{\ell} S \mathcal{F}
$$

as in Eq. (2.14) was obtained in Ref. [78]. It is shown in Fig. 5, together with its image under $u$ to the $u$ plane. We stress that (4.6) cannot be written as $G \backslash \mathbb{M}$ for any subgroup $G \subseteq \operatorname{SL}(2, \mathbb{Z})$.

In the massless $N_{f}=1$ theory, the partitioning (2.35) is contained in the algebraic plane curve $T_{(0)}(x, y)=0$, where $\frac{u}{\Lambda_{1}^{2}}=x+i y$ and

$$
\begin{aligned}
T_{(0)}(x, y)= & y\left(3 x^{2}-y^{2}\right)\left(27 x^{3}+128 x^{6}-81 x y^{2}\right. \\
& \left.+384 x^{4} y^{2}+384 x^{2} y^{4}+128 y^{6}\right) .
\end{aligned}
$$

The first two factors of $T_{(0)}(x, y)$ contains also values which correspond to $j>12^{3}$ and they need to be sufficiently truncated. The identification of the algebraic curve with the partitioning of $\mathbb{t}$ is immediate from Fig. 5.

\section{B. Type II AD mass}

In Sec. II C we saw that the $N_{f}=1$ theory has AD fixed points in its moduli space. To study these, we fix the mass to be one of the AD values, $m \rightarrow m_{\mathrm{AD}}=\frac{3}{4} \Lambda_{1}$, specified by the zero locus of the AD polynomials (2.24). Two mutually nonlocal singularities now collide at the AD point $u=$ $u_{\mathrm{AD}}=\frac{3}{4} \Lambda_{1}^{2}$ while the third one simplifies to $u_{0}=-\frac{15}{16} \Lambda_{1}^{2}$, such that the discriminant reads

$$
\Delta=\left(u-u_{\mathrm{AD}}\right)^{2}\left(u-u_{0}\right) .
$$



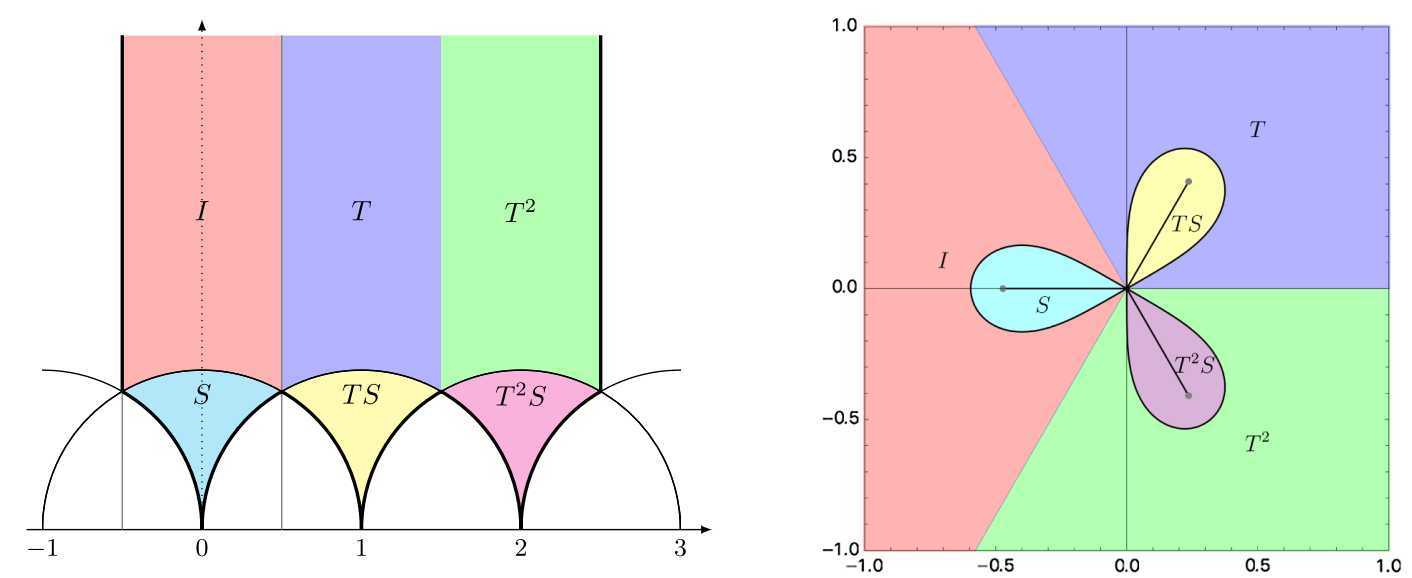

FIG. 5. Left: proposal of a fundamental region for massless $N_{f}=1$. It is clear that it can not be a fundamental domain of any SL(2, $\left.\mathbb{Z}\right)$ subgroup if we identify the sides, $\tau \sim \tau+3$. This is because the lower boundary is not given by a union of half disks. If we do not identify the sides then the picture is in fact a fundamental domain for $\Gamma^{0}(4)$. This is because there is an element in $\Gamma^{0}(4)$ which maps $i \infty \mapsto 1$. Right: plot of the massless $N_{f}=1 u$ plane as the union of the images of $u$ under the $\operatorname{six} \operatorname{SL}(2, \mathbb{Z})$ images of $\mathcal{F}$ as in $(4.6)$, in units of $\Lambda_{1}^{2}=1$. In particular, the function $u$ is surjective onto $\mathbb{C}$ on this domain. The singular points sit neatly in the interior of the strong coupling regions $u\left(S^{\ell} \mathcal{F}\right), \ell=0,1,2$. The origin $u=0$ is $\tau=\omega_{3}+\mathbb{Z}$ as discussed above, and bounds all six domains as is clear from the left figure.

From the curve we now find

$$
\frac{u(\tau)}{\Lambda_{1}^{2}}=-\frac{f_{3 \mathrm{~B}}(\tau)+15}{16}
$$

where

$$
\begin{aligned}
f_{3 \mathrm{~B}}(\tau) & =\left(\frac{\eta\left(\frac{\tau}{3}\right)}{\eta(\tau)}\right)^{12}=27\left(\frac{b_{3,0}\left(\frac{\tau}{3}\right)}{b_{3,1}\left(\frac{\tau}{3}\right)}\right)^{3}-27, \\
& =q^{-\frac{1}{3}}-12+54 q^{\frac{1}{3}}-76 q^{\frac{2}{3}}-243 q+\mathcal{O}\left(q^{\frac{4}{3}}\right)
\end{aligned}
$$

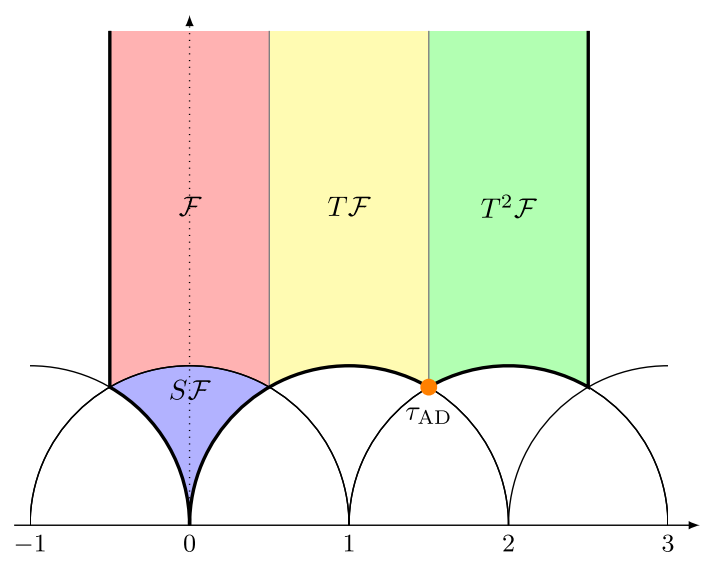

is the McKay-Thompson series of class 3B for the Monster group [80], and we are again careful to choose the solution for $u$ with the consistent asymptotics. Substitution of the $q$ series (4.10) in Eq. (4.9) reproduces the $q$ series based on [32] [Eq. (4.93)].

The functions $\eta$ and $b_{3, j}$ are defined in Appendix A 1. Using Theorem 1 in the same Appendix, we find that $\tau \mapsto f_{3 \mathrm{~B}}(3 \tau)$ is a classical modular function for $\Gamma_{0}(3)$ and therefore $u$ is a modular function for $\Gamma^{0}(3)$. A fundamental domain of $\Gamma^{0}(3)$ is

$$
\mathcal{F}_{1}\left(m_{\mathrm{AD}}\right)=\bigcup_{\ell=0}^{2} T^{\ell} \mathcal{F} \cup S \mathcal{F} .
$$

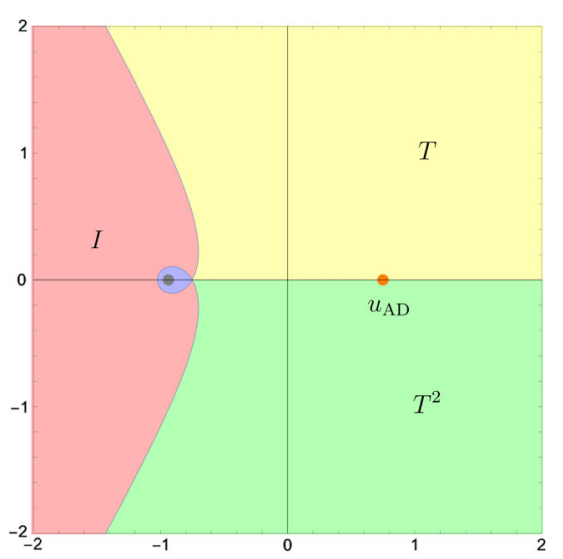

FIG. 6. Left: fundamental domain of $\Gamma^{0}(3)$, the duality group of $N_{f}=1$ with $m=m_{\mathrm{AD}}$. The AD point $\tau_{\mathrm{AD}}=\sqrt{3} \omega_{12}$ is highlighted. Right: plot of the $N_{f}=1 u$ plane with $\mathrm{AD}$ mass as the union of the images of $u$ under the $6-2=4 \operatorname{SL}(2, \mathbb{Z})$ images of $\mathcal{F}$ forming $\Gamma^{0}(3) \backslash \mathbb{H}$, as in the left figure. The complex plane can clearly be covered by four triangles. There is only one strong coupling region, which is the circular region. It contains $u_{0}$ in its interior. The AD point (orange) lies on the boundary of $T^{ \pm 1} \mathcal{F}$, as is clear from the left figure. The areas with the same colors are mapped to each other in the two figures. 
This is shown in Fig. 6 together with the map to the $u$ plane. The cusps are $i \infty$ and 0 , with widths 3 and 1, respectively. We take from [81] [Table 4.1] that $\Gamma^{0}(3)$ has an elliptic fixed point of order 3 .

Using the transformation properties of the $\eta$ function (A7) it is straightforward to show that the locations of the singularities of (4.8) in the $\tau$ plane are given by $\left(\omega_{j}=e^{2 \pi i / j}\right)$

$u(i \infty)=\infty, \quad u(0)=u_{0}, \quad u\left(\sqrt{3} \omega_{12}\right)=u_{\mathrm{AD}}$,

where the proper limits are understood. The AD point $\sqrt{3} \omega_{12}$ is stabilized by the order 3 element $\left(\begin{array}{l}-13 \\ -12\end{array}\right) \in$ $\Gamma^{0}(3)$, and it is therefore the order 3 elliptic fixed point of $\Gamma^{0}(3)$. Comparing the locations to the massless case we see that the regular singularity $u_{0}$ has stayed on $\tau=0$, while, contrary to the massless case, the cusps with the two mutually nonlocal singularities are disconnected (or cut) from the domain for massless $N_{f}=1$, and leaves as remnant the point $\tau_{\mathrm{AD}}$ into the interior of $\mathbb{\leftrightarrow}-{ }^{11}$ This procedure also reduces the index of the solution: indeed, from Eq. (A20) we compute that $\operatorname{ind} \Gamma^{0}(3)=4$, where the $6-4=2$ AD points do not contribute since they are not cusps. This can also be seen from the fact that

$$
j=\frac{\left(f_{3 \mathrm{~B}}+3\right)^{3}\left(f_{3 \mathrm{~B}}+27\right)}{f_{3 \mathrm{~B}}} .
$$

Indeed, since $\mathcal{J}=1728 \frac{g_{2}^{3}}{\Delta}$, a common factor $\left(f_{3 \mathrm{~B}}+27\right)^{2}$ of $g_{2}^{3}$ and $\Delta$ has canceled. The last factor $f_{3 \mathrm{~B}}+3=0$ in (4.13) implies $j(\tau)=0$ and therefore $\tau=\omega_{3} \bmod \operatorname{SL}(2, \mathbb{Z})$. In fact, it corresponds to $u\left(\omega_{3}\right)=u\left(\omega_{3}+1\right)=-\frac{3}{4} \Lambda_{1}^{2}$ and it is just a regular point in the $u$ plane. We can also read off from this that $\operatorname{ord}\left(g_{2}, g_{3}, \Delta\right)=(1,1,2)$ and therefore the AD theory in $N_{f}=1$ is according to Table III a type II singular fiber [82].

We can also study more characteristic functions of the theory with the AD mass. Using Appendix A 1, we can differentiate (4.9) to find

$$
\frac{d u}{d \tau}(\tau)=\frac{\pi i \Lambda_{1}^{2}}{24} f_{3 \mathrm{~B}}(\tau) b_{3,0}\left(\frac{\tau}{3}\right)^{2} .
$$

This implies that $\frac{d u}{d \tau}$ is a modular form of weight 2 for $\Gamma^{0}(3)$, without phases. One can also show that

$$
\frac{d a}{d u}(\tau)=\frac{\sqrt{2} i}{\sqrt{27} \Lambda_{1}} \sqrt{\frac{b_{3,1}\left(\frac{\tau}{3}\right)^{3}}{b_{3,0}\left(\frac{\tau}{3}\right)}} .
$$

An expression for $d a / d u$ in terms of ${ }_{2} F_{1}$ was given in Ref. [[83], Eq. (4.13)].

\footnotetext{
${ }^{11}$ The disconnected region has a physical interpretation as the $u$ plane of the AD curve. See the discussion around Eq. (4.20).
}

The $q$ expansion of $\frac{d a}{d u}$ has growing denominators, and therefore $\frac{d a}{d u}$ is not a modular form of weight 1 for $\Gamma^{0}(3)$. However, it is straightforward to check that $\left(\frac{d a}{d u}\right)^{2}$ is a modular form of weight 2 for $\Gamma^{0}(3)$. We thus find the Matone relation

$$
\frac{d u}{d \tau}=-\frac{16 \pi i}{3} \hat{\Delta}\left(\frac{d a}{d u}\right)^{2}
$$

where $\hat{\Delta}$ denotes the reduced discriminant. This is consistent with (3.13).

The monodromies can be found from the ones of the massless theory (4.3),

$$
\begin{aligned}
M_{1} & =S T S^{-1}=\left(\begin{array}{cc}
1 & 0 \\
-1 & 1
\end{array}\right), \\
M_{\mathrm{AD}} & =M_{2} M_{3}=T^{2}(S T)^{-1} T^{-2}=\left(\begin{array}{ll}
-1 & 3 \\
-1 & 2
\end{array}\right) .
\end{aligned}
$$

They generate the duality group $\Gamma^{0}(3)$ and give the large $u$ monodromy $M_{1} M_{\mathrm{AD}}=P T^{-3}$. Furthermore, $M_{1}$ stabilizes $\tau=0$ and $M_{\mathrm{AD}}$ stabilizes the AD point $\tau_{\mathrm{AD}}=\sqrt{3} \omega_{12}$. We have that $M_{\mathrm{AD}}^{6}=\mathbb{1}$ and therefore $\tau_{\mathrm{AD}}$ is indeed an elliptic fixed point. The $\mathrm{AD}$ monodromy is conjugate to $(S T)^{-1}$, which fixes $\tau=\omega_{3}$. Since $\tau_{\mathrm{AD}}=\omega_{3}+2$, this gives a path in $\tau$ space.

\section{Generic real mass}

By turning on a generic real mass, the singularities do not split compared to the massless case since there are already $N_{f}+2=3$ singularities. Therefore, the fundamental domain of the massive theory should look similar to the massless one of Fig. 5, but we now need to consider the presence of branch points and cuts in more detail. We will discuss this and the limits to the pure theory as well as the theory with the AD mass now.

For generic mass we have not been able to find a closed expression for $u$ as a function of $\tau$. By expanding $\mathcal{J}\left(u, m, \Lambda_{1}\right)$ and inverting the series we can, however, get an expansion of $u$ for the general massive theory near any cusp. For example, the expansion near $\tau=i \infty$ reads $\left(\mu=\frac{m}{\Lambda_{1}}\right)$

$$
\begin{aligned}
\frac{u(\tau)}{\Lambda_{1}^{2}}= & -\frac{1}{16} q^{-\frac{1}{3}}-\frac{1}{3} \mu^{2}+\left(\frac{32}{9} \mu^{4}-6 \mu\right) q^{\frac{1}{3}} \\
& -\left(\frac{5120}{81} \mu^{6}-\frac{160}{3} \mu^{3}+\frac{13}{2}\right) q^{\frac{2}{3}}+\mathcal{O}(q),
\end{aligned}
$$

where we are careful to choose the expansion such that $u \rightarrow-\infty$ for consistency with our conventions. It is easy to see that this reproduces the earlier expansions, (4.1) and (4.9), in the respective limits. 


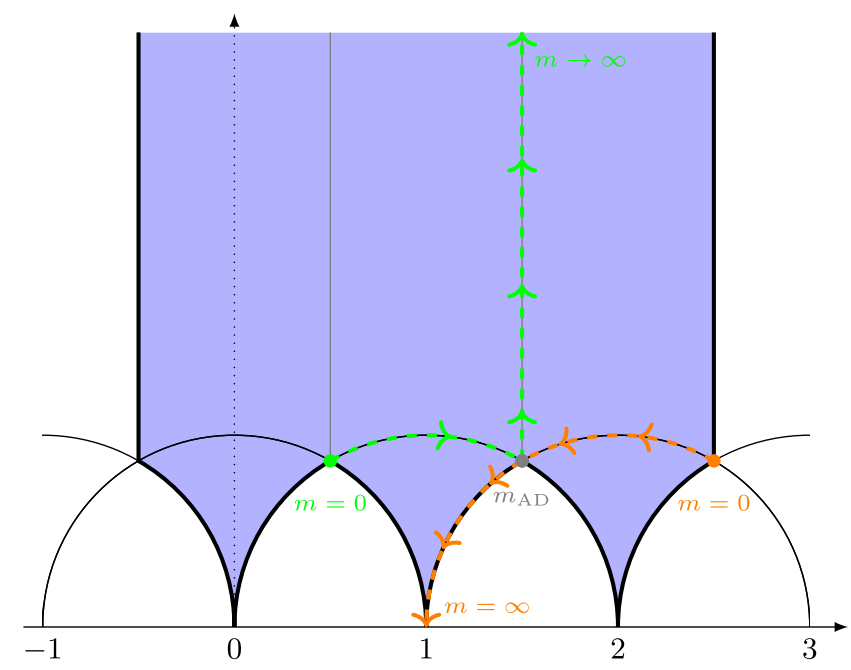

FIG. 7. Conjectured paths of the branch points in the fundamental domain of the massive $N_{f}=1$ theory.

The branch point locus is given by the zero locus of (2.28). By calculating $\mathcal{J}\left(u, m, \Lambda_{1}\right)$ from the curve and plugging it into the polynomial $D_{1}^{\mathrm{bp}}$ we find that the zero of the linear polynomial is $u=u_{\mathrm{bp}}=\frac{4}{3} m^{2}$, and we recognize that this is the polynomial appearing in the denominator of the generalized Matone relation, (3.15), such that $\frac{d u}{d \tau}$ diverges here. In the massive $N_{f}=2,3$ theories, where the theories can be studied in detail, we argue that it corresponds to two branch points in the closure of the fundamental domain, which are connected by a branch cut. Motivated by these analyses, we can draw the two branch point loci for positive mass. It is given in Fig. 7. For $m=0$, the branch point is located at the origin $u=0$. At the AD point, they collide, the branch cut vanishes and the order parameter becomes holomorphic, and even modular. In the $m \rightarrow \infty$ limit, the branch points also move to infinity.

We can also confirm this from the analysis in Sec. III C. By expanding $\mathcal{J}(u)-\mathcal{J}\left(u_{\mathrm{bp}}\right)$ around $u_{\mathrm{bp}}$ for generic mass $m$, the linear coefficient is zero. The $\left(u-u_{\mathrm{bp}}\right)^{2}$ coefficient vanishes if and only if either $m=0$ or $m=m_{i}:=\frac{3}{4 \sqrt[3]{2}}$. For $m=0$ we have $\tau_{\text {bp }} \sim \omega_{3}$, such that $n_{\omega_{3}}=3$ in the notation of Sec. III C. Furthermore, $n_{\mathrm{bp}}=6$, such that the order of the branch point (3.26) is the denominator of the reduced fraction $\frac{3}{6}$, namely 2 . Since $E_{4}$ has a simple zero at $\tau_{\mathrm{bp}}$, this agrees with Eq. (4.1) having a square root.

From Fig. 7 we see that the branch point loci pass through $\tau_{\mathrm{bp}}=1+i$ where $m=m_{i}$, such that $n_{i}=2$. Furthermore we find $n_{\mathrm{bp}}=4$, and thus the order of the branch point is 2 . Since $\frac{2}{4}=\frac{1}{2}$, it is indeed again the branch point of a square root.

For any other mass $m \in \mathbb{R}_{\geq 0} \backslash\left\{0, m_{i}, m_{\mathrm{AD}}\right\}$ we have $n_{\mathrm{bp}}=2$ while $n_{\tau_{\mathrm{bp}}}=1$, such that the branch point is again of order 2. This demonstrates that the loci in Fig. 7 are complete: there is a single branch point on the Coulomb branch $\mathcal{B}_{1}$, and for any mass there are two branch points of a square root in $\mathbb{U}$, which are connected by a single branch cut. It also implies that if an expression such as Eq. (4.1) existed for generic mass, while it could contain higher roots of modular forms, they can never have zeros in $\mathbb{U}$ (as is the case also for $m=0$ ).

We can study the partition of the $u$ plane provided by Eq. (2.35) in detail. For $m \neq m_{\mathrm{AD}}$ the $u$ plane is partitioned into six regions, whose union of boundary pieces is included in the algebraic curve given by the zero locus (2.37) of $T_{1}$, where $T_{1}=y \tilde{T}_{1}$ and

$$
\begin{aligned}
\tilde{T}_{1}= & 972 \mu^{4}+8192 \mu^{2} x^{7}-21504 \mu^{3} x^{5}-12096 \mu^{2} x^{4}+18432 \mu^{4} x^{3}+1296 \mu^{3} x^{2} \\
& +16128 \mu x^{6}+1944 \mu x^{3}+8192 \mu^{2} x^{5} y^{2}+22528 \mu^{3} x^{3} y^{2}-8192 \mu^{2} x^{3} y^{4}-3456 \mu^{2} x^{2} y^{2} \\
& +2304 \mu x^{4} y^{2}-6912 \mu x^{2} y^{4}-16384 x^{6} y^{2}-12288 x^{4} y^{4}+4320 x^{3} y^{2}-6144 x^{8} \\
& -1296 x^{5}-5184 \mu^{5} x-729 \mu^{2} x-18432 \mu^{4} x y^{2}-21504 \mu^{3} x y^{4}-8192 \mu^{2} x y^{6} \\
& -1944 \mu x y^{2}-1296 x y^{4}+4752 \mu^{3} y^{2}+8640 \mu^{2} y^{4}+6912 \mu y^{6}+2048 y^{8} .
\end{aligned}
$$

Since the AD point $m=m_{\mathrm{AD}}$ corresponds to a phase transition, we have to study the two cases $m<m_{\mathrm{AD}}$ and $m>m_{\mathrm{AD}}$ separately.

\section{The case $m<m_{\mathrm{AD}}$}

From Fig. 7 we can take the location of the branch points. There is one singularity $u_{1}$ on the negative real line, and the other two are complex conjugates (as $\Delta_{1}$ is a real polynomial). Using the definition (2.35), it is straightforward to show that not all of $y=0$ lies in $\mathcal{T}_{1}$, but rather only the real half-line with $u \geq u_{1}$. Furthermore, the lines truncate at the singularities. On the upper-half plane, the branch points can be viewed as end points of branch cuts coming from $\tau=\frac{1}{2}+\frac{\sqrt{3}}{2} i$ and $\tau=\frac{5}{2}+\frac{\sqrt{3}}{2} i$. See Fig. 8 . From this it is straightforward to see how taking the massless limit gives back Fig. 5.

\section{The case $m>m_{\mathrm{AD}}$}

At $m=m_{\mathrm{AD}}$ two singularities collide, and $\Delta_{1}$ has a double root. Since $\Delta_{1}$ is a real polynomial and depends 

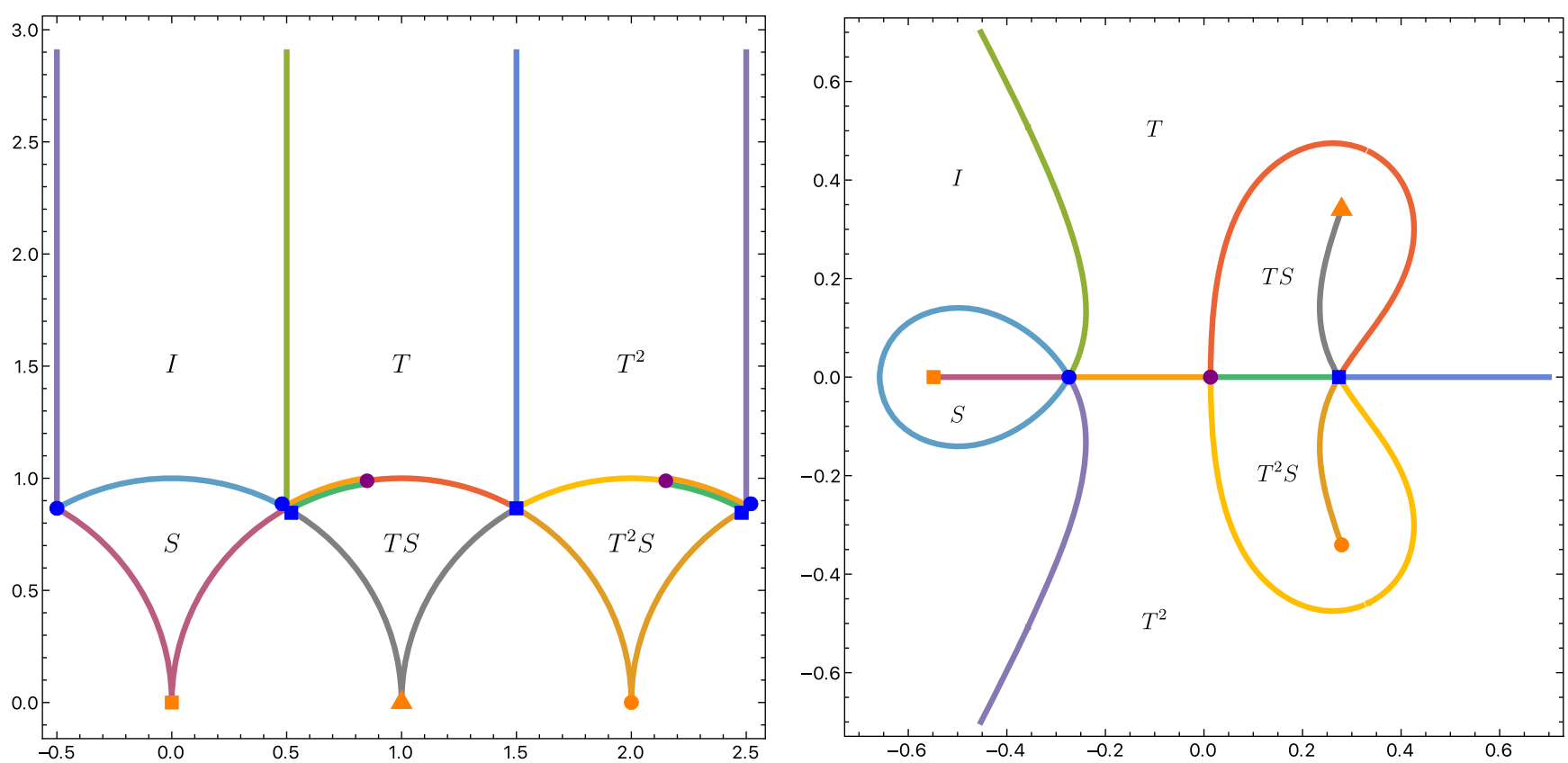

FIG. 8. Identification of the components of the partitioning $\mathcal{T}_{m}$ in $N_{f}=1$ for $\mu<\mu_{\mathrm{AD}}$, here for the choice $\mu=\frac{1}{10}$. The $u$-plane $\mathcal{B}_{1}$ is partitioned into six regions $u(\alpha \mathcal{F})$, with the $\alpha \in \mathrm{SL}(2, \mathbb{Z})$ given in both pictures. The branch point (purple) identifies two points on $\partial \mathcal{F}_{1}(m)$. The green/orange arcs are separated by a branch cut, and the opposite ends are glued with the corresponding identical color on the other side. The blue points correspond to $j=\mathcal{J}=0$ and form the intersection points of $\mathcal{T}_{1}$.

smoothly on $m$, the two singularities which are complex for $m<m_{\mathrm{AD}}$ are real for $m>m_{\mathrm{AD}}$. There is no meaningful identification of the singular points when going through $m=m_{\mathrm{AD}}$, however for large $m$ there is a distinguished

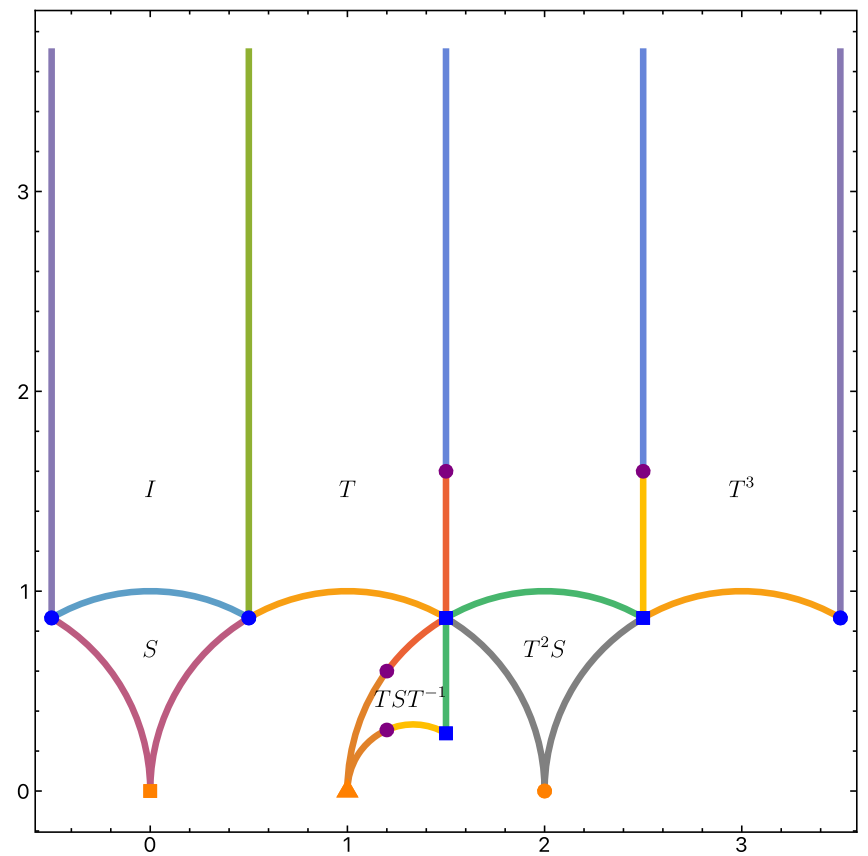

singularity $u_{*}$ that diverges. We can make the choice of $\mathcal{F}_{m}$ suitable for the limit $m \rightarrow \infty$, where we should obtain Fig. 2. By studying the dependence of the partition of the $u$ plane on the mass, one finds that $u_{*}$ is bounded by a region

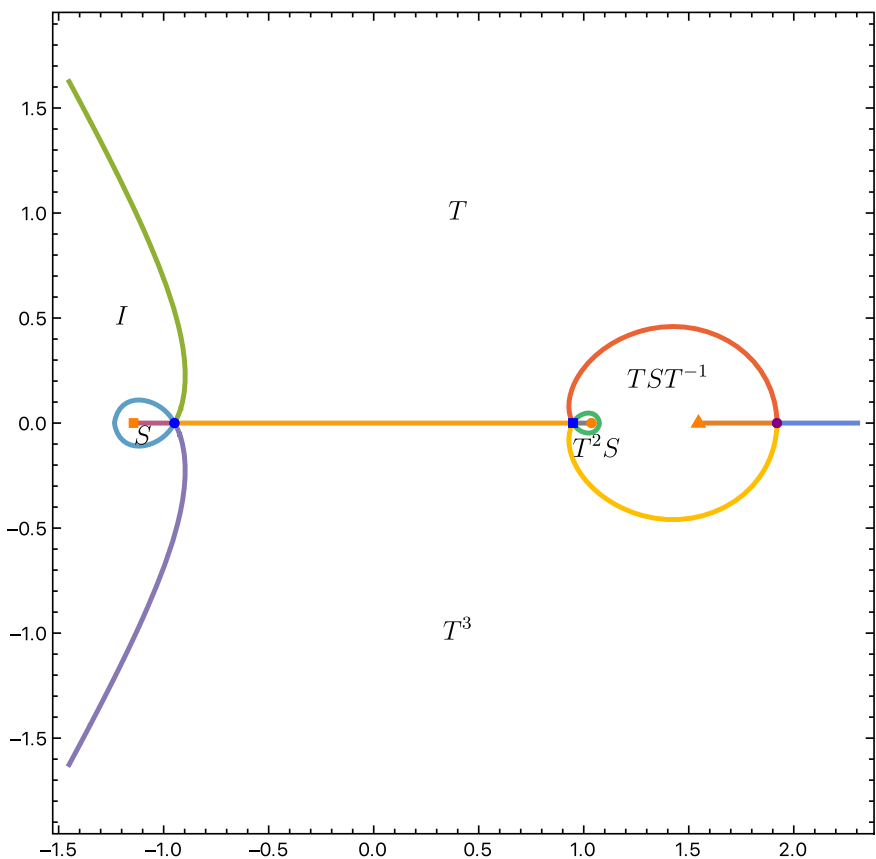

FIG. 9. Identification of the components of the partitioning $\mathcal{T}_{m}$ in $N_{f}=1$ for $\mu>\mu_{\mathrm{AD}}$, here for the choice $\mu=\frac{6}{5}$. The $u$-plane $\mathcal{B}_{1}$ is partitioned into six regions $u\left(\alpha_{j} \mathcal{F}\right)$, with the $\alpha_{j} \in \mathrm{SL}(2, \mathbb{Z})$ given in both pictures. The branch point (purple) identifies four points on $\partial \mathcal{F}_{1}(m)$. 
whose area grows as $m \rightarrow \infty$. It squeezes into $T \mathcal{F}$ and $T^{3} \mathcal{F}$ and becomes $T^{2} \mathcal{F}$ in the limit $m \rightarrow \infty$. However, as we want to put the singularities on the real line we need it to touch this axis for finite $m>m_{\mathrm{AD}}$. In order to find the corresponding fundamental domain, we can glue parts of the boundary $\partial \mathcal{F}_{1}$, such that it not only agrees with the geometry of the partition of the $u$ plane, but also the decoupling procedure is inherent. See Fig. 9.

An alternative way of depicting how the cuts change the fundamental domain is given in Fig. 10. Here, we lift the restriction that we want to have all the singularities situated on the real axis once we go to mass larger than the $\mathrm{AD}$ value. Figure 10 is equivalent to Figs. 8 and 9, as is easily seen by following the identification of the various boundaries. From this description it is direct to see the change in the domain for the different special limits of the mass.

\section{The case $m=m_{\mathrm{AD}}$}

Finally, let us return to the case $m=m_{\mathrm{AD}}$ discussed in detail in Sec. IV B. As explained above, a region is

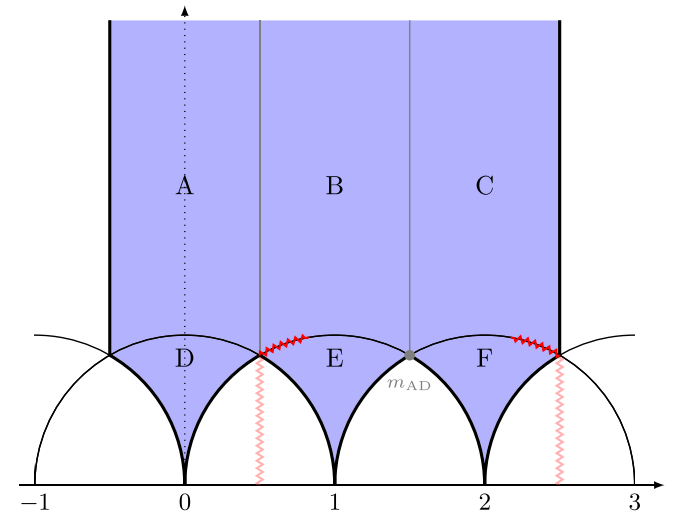

(a)

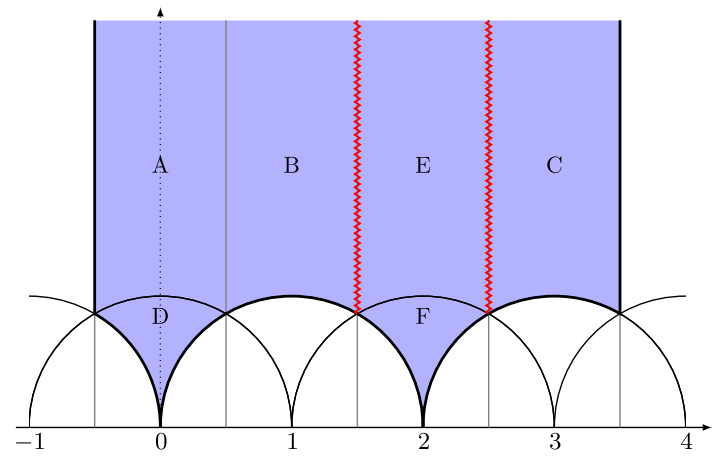

(c) disconnected from $\mathcal{F}_{N_{f}}$ for this special value of the mass. The disconnected domain is labeled by $T S$ and $T^{2} S$ in Fig. 8, $T S T^{-1}$ and $T^{2} S$ in Fig. 9, and by $E+F$ in Fig. 10. It is also isomorphic to the domain in Fig. 20. This region has a physical meaning, namely as the fundamental domain for the order parameter $\tilde{u}$ of the $\mathrm{AD}$ theory, obtained after taking the scaling limit to the conformal field theory. To see this, recall that the scaling limit brings the $N_{f}=1$ curve (2.3) to the AD curve $[33,34]$

$$
y^{2}=x^{3}-\frac{1}{4} \Lambda_{1}^{3} \tilde{m} x-\frac{1}{16} \Lambda_{1}^{4} \tilde{u},
$$

which gives for $\tilde{u}$ in terms of the effective coupling $\tau$ [18],

$$
\tilde{u}(\tau)=\frac{4}{\sqrt{27}} \Lambda_{1}^{1 / 2} \tilde{m}^{3 / 2} \frac{E_{6}(\tau)}{E_{4}(\tau)^{3 / 2}},
$$

for which the disconnected domain is indeed a fundamental domain. This splitting of the fundamental domain $\mathcal{F}_{1}$ at the

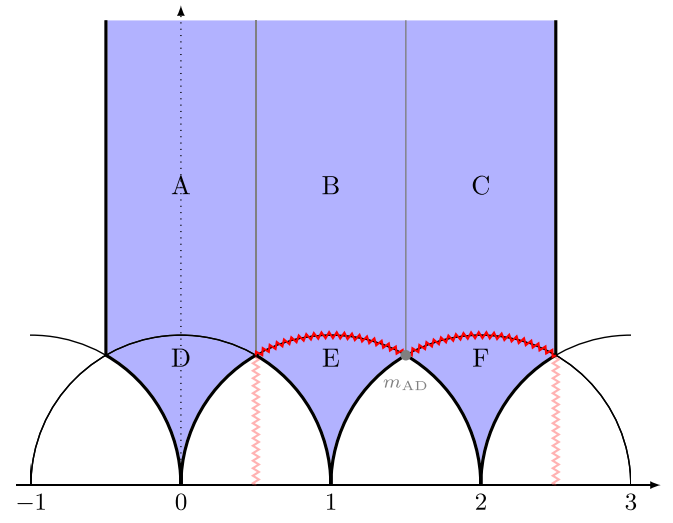

(b)

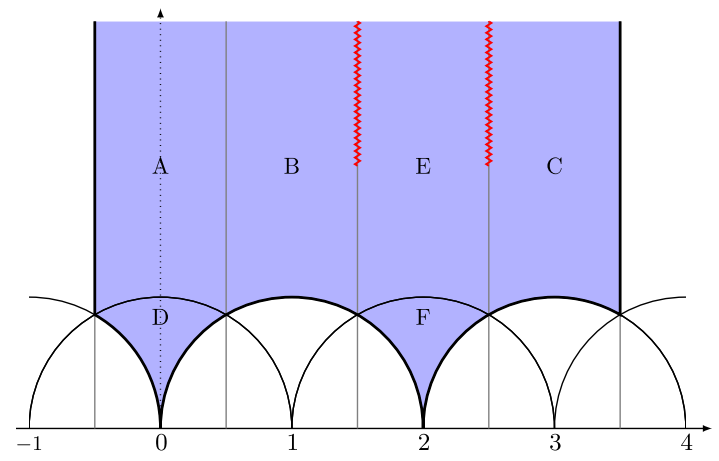

(d)

FIG. 10. Choice of branch cuts (red zigzag lines) for varying mass in $N_{f}=1$. Starting with a small mass in (a) we cut along the paths shown in Fig. 7. At the AD mass we can use the identifications of the different boundaries to reorganize the domain in (b) to the one of (c). In (b) we identify the upper part of the cut in region B with the upper part of the cut in region C, and similarly in E and F. In (c) we instead identify the sides of the cuts such that the boundaries of regions B and C are identified and the two boundaries of E are identified. When we increase the mass further the cuts of (c) move upwards as in (d) eventually reaching infinity and disappearing, leaving us with the domain of the pure theory. 

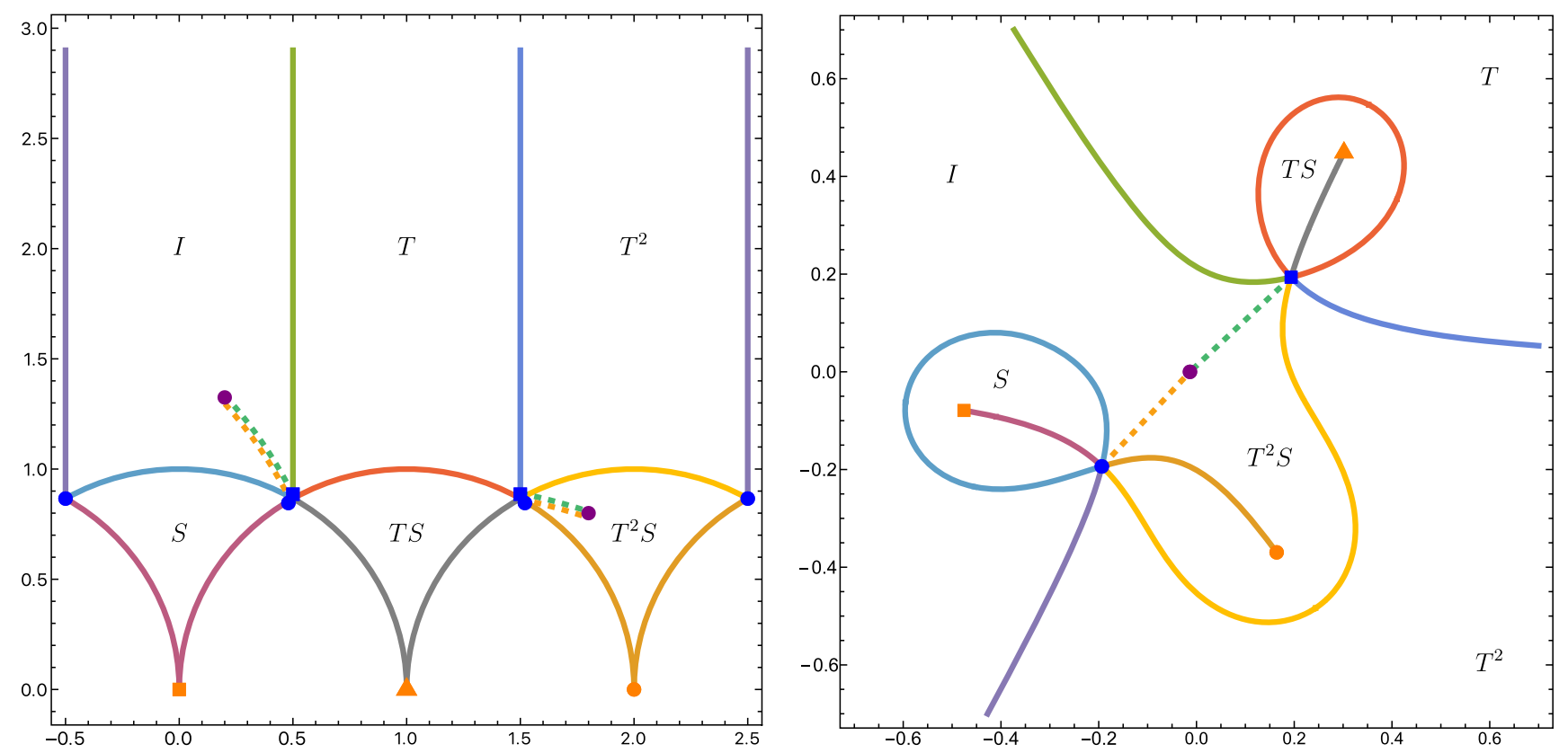

FIG. 11. Identification of the components of the partitioning $\mathcal{T}_{m}$ in $N_{f}=1$ for a complex mass $\mu \in \mathbb{C}$, here for the choice $\mu=\frac{i}{10}$. The $u$-plane $\mathcal{B}_{1}$ is partitioned into five regions, which is due to the fact that $u(\mathcal{F})$ and $u\left(T^{2} S \mathcal{F}\right)$ are glued at the branch cuts $($ dashed). The purple dot is the branch point in $N_{f}=1$.

AD point appears to fit well with the $u$-plane integral for $N_{f}=1$ discussed in [18].

\section{Generic complex mass}

We can also consider a generic complex mass. The locus of AD masses (2.24) is then real codimension 2. In fact, it is just $\omega_{3} m_{\mathrm{AD}}$, with $\omega_{3}$ a cube root of unity. If $m$ is not any of these three values, then the corresponding Coulomb branch has three distinct singularities.

We can decompose $m=a+i b$, and $T_{m}$ is then a polynomial in $a, b, x$, and $y$. From Eq. (2.28) we see that if $m$ is complex, then $j\left(\tau_{\mathrm{bp}}\right)=\mathcal{J}\left(u_{\mathrm{bp}}\right)$ is also complex, such that $\tau_{\mathrm{bp}}$ is generically an interior point of $\mathcal{F}$ or an $\mathrm{SL}(2, \mathbb{Z})$ copy thereof. The branch cuts most conveniently run from such branch points to the intersection points of the curves, where $\mathcal{J}(u)=j(\tau)=0$. From Eq. (2.9) it is clear that they correspond to the two solutions of $g_{2}(u)=0$. We plot the partitioning of the $u$ plane with the branch cuts for an imaginary mass in Fig. 11.

Due to the fact that $u_{\mathrm{bp}} \notin \mathcal{T}_{1}$, the branch cuts run to the interior of the $\alpha_{j} \mathcal{F}$. The four sides of the two cuts are pairwise identified, which makes points on the branch cut smooth points on the Riemann surface. This identification glues different regions $u\left(\alpha_{j} \mathcal{F}\right)$ together, in this case $u(\mathcal{F})$ and $u\left(T^{2} S \mathcal{F}\right)$. This is clearly visible in Fig. 11, where the dashed lines in the $u$ planes are the images of the branch cuts in $\mathcal{F}_{m}$, and they do not belong to the partitioning $\mathcal{T}_{1}$. As a result, the $u$ plane is partitioned into five and not six components. This is not in contradiction with Sec. II because the fundamental domain $\mathcal{F}_{1}(m)$ is still a union of six copies of $\mathcal{F}$ : the cutting and gluing along the branch cuts is an additional feature of the domain.

\section{THE $N_{f}=2$ CURVE}

Let us now move on to discuss the theory with two hypermultiplets. This theory has four strong coupling singularities where massless hypermultiplets appear. For general masses they are distinct points while for special mass configurations one or more singularities can collide. We will begin by restricting to the case of equal masses, $m_{1}=m_{2}=m$, where we can find explicit expressions for $u$ as a function of $\tau$. Then we briefly discuss the case of two distinct masses before moving on to discuss what happens in the simpler cases of massless hypermultiplets and when fixing the mass to an $\mathrm{AD}$ value.

\section{A. Equal masses}

Let us consider first the equal mass case, $\boldsymbol{m}=(m, m)$, where $m \neq 0$ and $m \neq m_{\mathrm{AD}}=\frac{1}{2} \Lambda_{2}$. It is discussed in detail in Ref. [84]. In this case, the discriminant factors as

$$
\Delta=\left(u-u_{*}\right)^{2}\left(u-u_{+}\right)\left(u-u_{-}\right),
$$

where $u_{*}=m^{2}+\frac{\Lambda_{2}^{2}}{8}$ and $u_{ \pm}=-\frac{\Lambda_{2}^{2}}{8} \pm m \Lambda_{2}$. It is easy to check that $\left\{u_{*}, u_{+}, u_{-}\right\}$never collide other than in the two cases mentioned above. Using the modular lambda function, $\lambda=\frac{\vartheta_{2}^{4}}{\vartheta_{3}^{4}}$, as a generator of the intermediate field $\Gamma(2)$, 
the sextic equation factors into three quadratic polynomials over $\Gamma(2)$. These equations can now be solved exactly. In $N_{f}=2$, two solutions have the property that $|u(\tau)| \rightarrow \infty$ when $\tau \rightarrow i \infty$. Following our (usual) convention, we choose

$$
\begin{aligned}
\frac{u}{\Lambda_{2}^{2}}= & -\frac{\vartheta_{4}^{8}+\vartheta_{2}^{4} \vartheta_{3}^{4}+\left(\vartheta_{2}^{4}+\vartheta_{3}^{4}\right) \sqrt{16 \frac{m^{2}}{\Lambda_{2}^{2}} \vartheta_{2}^{4} \vartheta_{3}^{4}+\vartheta_{4}^{8}}}{8 \vartheta_{2}^{4} \vartheta_{3}^{4}}, \\
= & -\frac{1}{64} q^{-1 / 2}-\frac{m^{2}}{\Lambda_{2}^{2}}+\left(64 \frac{m^{4}}{\Lambda_{2}^{4}}-32 \frac{m^{2}}{\Lambda_{2}^{2}}-\frac{5}{16}\right) q^{1 / 2} \\
& +\mathcal{O}(q) .
\end{aligned}
$$

Due to the appearance of the square root in Eq. (5.2) $u$ is not holomorphic, and similarly to the $N_{f}=1$ case there will be branch points in the fundamental domain. From Sec. II C we expect them to be given by

$$
j^{\mathrm{bp}}(m)=16 \frac{\left(16 m^{2}-\Lambda_{2}^{2}\right)^{3}}{m^{2}} .
$$

By plugging in the solution for $\mathcal{J}\left(u, m, \Lambda_{2}\right)$ we find that this corresponds to $u=u_{\mathrm{bp}}=2 m^{2}-\frac{\Lambda_{2}^{2}}{8}$. We recognize this as the root of the polynomial $P_{2}^{M}$ of the generalized Matone relation. By using standard relations between the $j$ invariant and Jacobi theta function we can also check that this coincides with the zeros of the square root.

Defining $f_{2}(\tau):=16 \frac{m^{2}}{\Lambda_{2}^{2}} \vartheta_{2}(\tau)^{4} \vartheta_{3}(\tau)^{4}+\vartheta_{4}(\tau)^{8}$, we see that the branch point of the square root is $f_{2}\left(\tau_{0}\right)=0$. Near $\tau_{0}$, the expansion of $f_{2}$ reads $f_{2}(\tau)=\left(\tau-\tau_{0}\right) h(\tau)$, where $h(\tau)$ is holomorphic near $\tau_{0}$ and $h\left(\tau_{0}\right) \neq 0$. Then one branch of the square root reads $\sqrt{f_{2}(\tau)}=\sqrt{\tau-\tau_{0}} \sqrt{h(\tau)}$. Now since $h\left(\tau_{0}\right) \neq 0$, we have that $\tau \mapsto \sqrt{h(\tau)}$ is nonzero and in fact holomorphic in a neighborhood of $\tau_{0}$. However, $\tau \mapsto \sqrt{\tau-\tau_{0}}$ is strictly nonholomorphic at $\tau_{0}$. This proves that $u$ is not holomorphic at $\tau_{0}$.

From Eq. (5.2) we can also calculate the other interesting quantities,

$$
\begin{aligned}
& \frac{d a}{d u}=-\frac{i}{\Lambda_{2}} \frac{\vartheta_{2}^{2} \vartheta_{3}^{2}}{\sqrt{\vartheta_{2}^{4}+\vartheta_{3}^{4}+\sqrt{f_{2}}}}, \\
& \frac{d u}{d \tau}=\pi i \Lambda_{2}^{2} \vartheta_{4}^{8} \frac{2\left(4 \frac{m^{2}}{\Lambda_{2}^{2}}+1\right) \vartheta_{2}^{4} \vartheta_{3}^{4}+\vartheta_{4}^{8}+\left(\vartheta_{2}^{4}+\vartheta_{3}^{4}\right) \sqrt{f_{2}}}{8 \vartheta_{2}^{4} \vartheta_{3}^{4} \sqrt{f_{2}}} .
\end{aligned}
$$

We can again explicitly check that they satisfy Matone's relation, (3.13),

$$
\frac{d u}{d \tau}=-\frac{16 \pi i}{2} \frac{\hat{\Delta}}{u-u_{\mathrm{bp}}}\left(\frac{d a}{d u}\right)^{2}
$$

On the rhs, the double singularity $u_{*}$ has canceled, while, as discussed in Sec. III, the branch point $u_{\mathrm{bp}}=2 m^{2}-\frac{1}{8} \Lambda_{2}^{2}$ remains in the denominator.

\section{Fundamental domain}

A fundamental domain can be found in the following way. The six roots of the sextic equation gives the six cusp expansions. In order to simplify the expressions, let us momentarily set $\Lambda_{2}=1$ and $(a, b, c):=\left(\vartheta_{2}^{4}, \vartheta_{3}^{4}, \vartheta_{4}^{4}\right)$. All six expressions can be brought to a canonical form, see Table I.

The overall sign can be fixed from the purely quadratic term in the numerator. Using the Jacobi identity $a+c=b$, such a representation is unique and the expressions cannot be further simplified. Then instead of studying which transformations give the right values at the cusps, we can take the cusp expansions and try to find maps $\alpha_{j} \in$ $\operatorname{SL}(2, \mathbb{Z})$ that takes $u(\tau)$ to the functions under study. Due to the square root, this is very subtle. For instance, for $T^{2} S T$ the Jacobi theta functions transform as $(a, b, c) \mapsto$ $\left(e^{2 \pi i} a, b, c\right) \mapsto\left(e^{2 \pi i} c, b, a\right) \mapsto\left(e^{2 \pi i} b, c, e^{\pi i} a\right)$. We ignore the weight factors since numerator and denominator are homogeneous in the modular weight. This implies that

$$
\begin{aligned}
\sqrt{c^{2}+16 m^{2} a b} & \mapsto \sqrt{e^{2 \pi i} a^{2}+16 m^{2} e^{2 \pi i} b c} \\
& =-\sqrt{a^{2}+16 m^{2} b c},
\end{aligned}
$$

and gives precisely the last row in Table I. The other transformations can also be proven directly. Such identifications are valid as long as $m$ is generic, and in particular such that the square root does not resolve. This obviously excludes the cases $m=0$ and $m= \pm m_{\mathrm{AD}}$, and it is conceivable that these are the only cases. We continue by assuming that it is true.

As argued above, there will also be branch points in the fundamental domain due to the square roots appearing in the solution for $u$. For generic complex mass these points will lie inside the fundamental domain. If we restrict to

TABLE I. Cusp expansions and associated coset representatives $\alpha_{j}$ of the solution for $N_{f}=2$ with mass $\boldsymbol{m}=(m, m)$.

\begin{tabular}{lcc}
\hline \hline$\alpha_{j}$ & $\alpha_{j}(i \infty)$ & $\alpha_{j} u$ \\
\hline $\mathrm{id}$ & $i \infty$ & $-\frac{c^{2}+a b+(a+b) \sqrt{c^{2}+16 m^{2} a b}}{8 a b}$ \\
$T$ & $i \infty$ & $-\frac{b^{2}-a c+(-a+c) \sqrt{b^{2}-16 m^{2} a c}}{-8 a c}$ \\
$S$ & 0 & $-\frac{a^{2}+b c+(b+c) \sqrt{a^{2}+16 m^{2} b c}}{8 b c}$ \\
$T S$ & 1 & $-\frac{b^{2}-a c+(a-c) \sqrt{b^{2}-16 m^{2} a c}}{-8 a c}$ \\
$T S T^{-1}$ & 1 & $-\frac{c^{2}+a b+(-a-b) \sqrt{c^{2}+16 m^{2} a b}}{8 a b}$ \\
$T^{2} S T$ & 2 & $-\frac{a^{2}+b c+(-b-c) \sqrt{a^{2}+16 m^{2} b c}}{8 b c}$ \\
\hline \hline
\end{tabular}




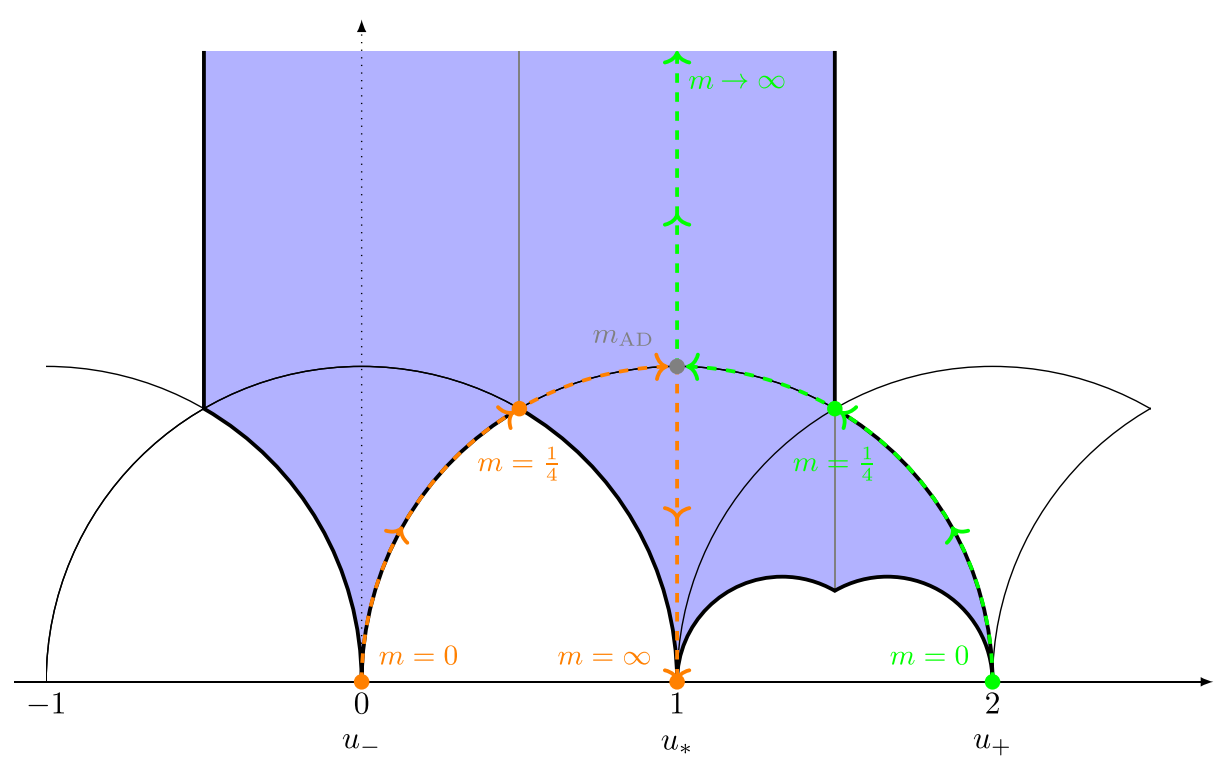

FIG. 12. Fundamental domain $\mathcal{F}_{2}(m, m)$ of massive $\boldsymbol{m}=(m, m) N_{f}=2$ theory. The dashed lines correspond to the conjectured paths of the branch points from zero to infinite mass. For a given positive mass $m$, the two branch points are identified under $T S T^{-1}$, such that there is only one branch point $u_{\mathrm{bp}}=2 m^{2}-\frac{\Lambda_{2}^{2}}{8}$ on the $u$ plane. At $m=m_{\mathrm{AD}}$ the two branch points meet, the square root in $u(\tau)$ resolves and $u(\tau)$ becomes holomorphic and modular.

positive masses we see from (5.3) that $\lim _{m \searrow 0} j^{\mathrm{bp}}(m)=-\infty$, while $j^{\mathrm{bp}}\left(\frac{\Lambda_{2}}{4}\right)=0, j^{\mathrm{bp}}\left(m_{\mathrm{AD}}\right)=12^{3} \Lambda_{2}^{4}$ with $m_{\mathrm{AD}}=\frac{1}{2} \Lambda_{2}$ and $\lim _{m \rightarrow \infty} j^{\mathrm{bp}}(m)=+\infty$. Furthermore, one finds that $j^{\text {bp }}:(0, \infty) \rightarrow \mathbb{R}$ is monotonically increasing, and $\mathbb{R}$ is partitioned into $j^{\mathrm{bp}}\left(\left(0, \frac{\Lambda_{2}}{4}\right]\right)=(-\infty, 0), \quad j^{\mathrm{bp}}\left(\left[\frac{\Lambda_{2}}{4}, \frac{\Lambda_{2}}{2}\right]\right)=$ $\left[0,12^{3} \Lambda_{2}^{4}\right]$ and $j^{\text {bp }}\left(\left[\frac{\Lambda_{2}}{2}, \infty\right)\right)=\left[12^{3} \Lambda_{2}^{4}, \infty\right)$. We aim to find a curve in $\tau$ space with these properties.

The branch point is located at $u=u_{\mathrm{bp}}=2 m^{2}-\frac{\Lambda_{2}^{2}}{8}$. In the case $m=0, u_{\mathrm{bp}}=u_{+}=u_{-}$collide. For $m=\frac{\Lambda_{2}}{4}$, the branch point $u_{\mathrm{bp}}=0$ is at the origin. At the $\mathrm{AD}$ mass $m=\frac{\Lambda_{2}}{2}$, the branch point collides with $u_{*}$ and $u_{+}$at $\tau_{\mathrm{AD}}=$ $1+i$ (see Fig. 16). We can use this knowledge to conjecture the branch point paths in $\tau$ space.

The cosets that we found above allow to construct a fundamental domain

$$
\mathcal{F}_{2}(m, m)=\mathcal{F} \cup T \mathcal{F} \cup S \mathcal{F} \cup T S \mathcal{F} \cup T S T^{-1} \mathcal{F} \cup T^{2} S T \mathcal{F},
$$

where we take the union of the elements in Table I. This is drawn in Fig. 12 together with the conjectured paths of the branch points. Since the $\alpha_{j}$ generate the whole $\operatorname{SL}(2, \mathbb{Z})$, it is clear that this domain is not a fundamental domain of any congruence subgroup of $\operatorname{SL}(2, \mathbb{Z})$. By computing the $q$ series of all the cusp expansions, one can match the singularities with the cusps,

$$
u(0)=u_{-}, \quad u(1)=u_{*}, \quad u(2)=u_{+} .
$$

The generic mass case $\boldsymbol{m}=\left(m_{1}, m_{2}\right)$ splits the singularity $u_{*}$ further and removes either $T S \mathcal{F}$ or $T S T^{-1} \mathcal{F}$ away from $\tau=1$.

Let us give some further evidence for the paths of the branch points. The points for $m=0, m=m_{\mathrm{AD}}$ and $m=\infty$ are fixed from the fact that in all three limits the duality group of the theory becomes a congruence subgroup. The branch points approach either $\tau=1$ or $i \infty$ in the decoupling limit, since these are identified under $\Gamma^{0}(4)$. This agrees with the fact that $u_{\mathrm{bp}} \rightarrow \infty$ for $m \rightarrow \infty$. We can also check it against the solution (5.2). The branch point satisfies $f_{2}=0$, for which $u$ simplifies,

$$
\begin{aligned}
\frac{u\left(\tau_{\mathrm{bp}}\right)}{\Lambda_{2}^{2}} & =-\frac{f_{2 \mathrm{~B}}\left(\frac{\tau_{\mathrm{bp}}}{2}\right)+16}{128}, \\
f_{2 \mathrm{~B}}(\tau) & =\left(\frac{\eta(\tau)}{\eta(2 \tau)}\right)^{24}=256 \frac{\vartheta_{3}(\tau)^{4} \vartheta_{4}(\tau)^{4}}{\vartheta_{2}(\tau)^{8}},
\end{aligned}
$$

where $f_{2 \mathrm{~B}}$ is a Hauptmodul of the congruence subgroup $\Gamma_{0}(2) \subseteq \mathrm{SL}(2, \mathbb{Z})$. One can plot this $u$ over the curves given in Fig. 12, and not only find that it is real everywhere, but it behaves as $u_{\mathrm{bp}}=2 m^{2}-\frac{\Lambda_{2}^{2}}{8}$ as a function of $m$. In particular, it is monotonically increasing and has the correct intermediate and limiting points $m \in\left\{0, \frac{\Lambda_{2}}{4}, \frac{\Lambda_{2}}{2}, \infty\right\}$. Therefore, the curves in Fig. 12 are parametrizations of (5.3) compatible with our solution for $u$.

For any mass, the pair of branch points is identified under $u$. In order to see this, note that the value of $u$ at a branch point is given by (5.9). Since it is a modular function 
for $\Gamma^{0}(2)$, it is invariant under $T S T^{-1}$. This relates the two loci in Fig. 12 at both smooth components of each curve,

$$
T S T^{-1}:\left\{\begin{array}{lll}
1+e^{\varphi i} & \longmapsto & 1+e^{(\pi-\varphi) i} \\
1+i \delta & \longmapsto & 1+\frac{1}{\delta} i
\end{array} .\right.
$$

The pair of two such points are the branch points of the square root, and the branch cut can be any path connecting the two branch points [85]. For $m>m_{\mathrm{AD}}$ for instance, on can take it to be the complex interval $\mathcal{I}_{\delta}=1+\left[\frac{1}{\delta}, \delta\right] i$. This can also be seen from the fact that when $\tau$ traverses a small circle around one branch point, the expression $u(\tau)$ receives a minus sign in front of the square root. According to Table I this interchanges the cusp expansions in the regions $T \mathcal{F}$ and $T S \mathcal{F}$, and the transition map is precisely $(T S) T^{-1}$ as in Eq. (5.10). For $m<m_{\mathrm{AD}}$ the branch points sit on the boundaries of $S \mathcal{F}$ and $T^{2} S T \mathcal{F}$, and the transition map $S\left(T^{2} S T\right)^{-1}=T S T^{-1}$ is identical. In order to achieve single valuedness, any path encircling one branch point must also encircle the other. On a dog bone contour around the interval $\mathcal{I}_{\delta}$ the function $u(\tau)$ returns to the original value, as it picks up twice the phase factor -1 . The function $u(\tau)$ is then a continuous single-valued function on the slit plane $\mathcal{F}(m, m) \backslash \mathcal{I}_{\delta}$, which one may interpret as a Riemann surface.

\section{Limits to zero, $A D$ and infinite mass}

The limits to other theories are given as follows. For $m \rightarrow 0$, the singularities $u_{+}$and $u_{-}$merge at $-\frac{\Lambda_{2}^{2}}{2^{8}}$, which we located at $\tau=0$. This agrees with the fact that for $m=0$ the order parameter is modular for $\Gamma(2)$ and in particular invariant under $T^{2}$. More precisely, we can use $\Gamma(2)$ to move the copies $T S T^{-1} \mathcal{F}$ and $T^{2} S T \mathcal{F}$ in order to obtain a more canonical form of $\Gamma(2) \backslash \mathrm{SL}(2, \mathbb{Z})$. For this, note that we can identify $S T^{-1} \mathcal{F}$ and $T^{2} S T \mathcal{F}$, since

$S T^{-1}\left(T^{2} S T\right)^{-1}=\left(\begin{array}{cc}1 & -2 \\ 2 & -3\end{array}\right) \in \Gamma(2): 2 \longmapsto 0$.

Similarly, we can identify $T S T^{-1} \mathcal{F}$ with $T S T \mathcal{F}$, as the transition function is also in $\Gamma(2)$. This gives precisely Fig. 15. In fact, since these transition functions are in $\Gamma(2)$, Figure 12 gives an equivalent fundamental domain for $\Gamma(2)$. It is however not the preferred choice for two reasons. First, not all copies of $\mathcal{F}$ are in a strip of width 2. Second, not all possibilities for cusp identifications have been taken, and it is preferable to only show inequivalent cusps. It is noteworthy that the (horizontal) width of the domain for fixed imaginary part never exceeds 2 , and that it is possible to draw the fundamental domain as an actual domain, i.e. a connected open subset of $\mathbb{H}$.

The decoupling limit $m \rightarrow \infty$ to $N_{f}=0$ is also interesting. The triangle $T S T^{-1} \mathcal{F}$ can be identified with $T^{2} \mathcal{F}$ since
$\left(T^{2}\right)^{-1} T S T^{-1}=\left(\begin{array}{cc}-1 & 0 \\ 1 & -1\end{array}\right) \in \Gamma^{0}(4): 1 \longmapsto i \infty$.

Similarly, we can identify $T S \mathcal{F}$ with $T^{3} \mathcal{F}$ as the transition map is in $\Gamma^{0}(4)$ and also maps $1 \mapsto i \infty$. Lastly, the triangle $T^{2} S T \mathcal{F}$ around $\tau=0$ can be identified with $T^{2} S \mathcal{F}$. This demonstrates that not only do we get the domain $\Gamma^{0}(4)$ as in Fig. 2, but in fact the domain in Fig. 12 is also a fundamental domain for $\Gamma^{0}(4)$. Aside from the disclaimer of the above paragraph, it is not a sound modular domain for $\Gamma^{0}(4)$ as the lines with constant real parts are not identified. The flow to the low energy effective theory with no hypermultiplets can be understood from the modular curve perspective as identifying the cusp $\tau=1$ of width 2 with the cusp $i \infty$, such that the number of rational cusps decreases by 2 , while the width of the cusp $i \infty$ increases by 2 .

In the $\mathrm{AD}$ limit $m \rightarrow \pm m_{\mathrm{AD}}$, the mutually nonlocal singularities $u_{*}$ and $u_{+}$collide and become elliptic points of the curve. This eliminates all the triangles near these cusps: in this case the regions $T S \mathcal{F}, T S T^{-1} \mathcal{F}$ and $T^{2} S T \mathcal{F}$ are removed and the domain of the theory with this mass, see Fig. 16, remains. The AD point $\tau_{\mathrm{AD}}$ lies in the interior of $\mathbb{\sharp}$, and is an elliptic point of the duality group $\Gamma^{0}(2)$.

\section{3. $u$ plane of $A D$ theory}

Similarly to the AD point for $N_{f}=2$, the disconnected cusps corresponding to the nonlocal singularities form the fundamental domain for order parameter of the $\mathrm{AD}$ curve. The disconnected cusps form a fundamental domain for $\Gamma_{0}(2)$, which is incidentally congruent to the duality group of the asymptotically free theory at the AD point. To demonstrate this, recall that the AD curve reads [34],

$$
y^{2}=x^{3}-\frac{\Lambda_{2}^{2}}{4} \tilde{u} x-\frac{\Lambda_{2}^{3}}{12} m \tilde{u}+\frac{\Lambda_{2}^{3}}{27} m^{3} .
$$

This gives for the order parameter

$$
\tilde{u}(\tau)=\frac{4}{3} m^{2}-\frac{m^{2} f_{2 B}(\tau)}{64+f_{2 B}(\tau)},
$$

with $f_{2 B}(\tau)$ as in (5.9). $f_{2 B}$ is a Hauptmodul for $\Gamma_{0}(2)$, such that the disconnected domain is indeed a fundamental domain for $\tilde{u}$.

\section{Partitioning of the $u$ plane}

Finally, we can study the partitioning that the domain (5.7) induces on the $u$ plane under the map (5.2). As studied in Sec. II D, the partitioning is contained in a real algebraic plane curve, which is given by the equation $\operatorname{Im} \mathcal{J}\left(u, \boldsymbol{m}, \Lambda_{2}\right)=0$. For generic $\mu=\frac{m}{\Lambda_{2}}$, we can compute it as the zero locus of the polynomial 


$$
\begin{aligned}
T_{(m, m)}= & y\left(-128 \mu^{2} x+48 \mu^{2}+64 x^{2}-16 x+64 y^{2}-3\right) \\
& \times\left(-720896 \mu^{4} x^{2} y^{2}+262144 \mu^{2} x^{2} y^{4}-262144 \mu^{2} x^{4} y^{2}-262144 \mu^{2} x^{3} y^{2}\right. \\
& -303104 \mu^{2} x^{2} y^{2}-589824 \mu^{6} x^{2}+688128 \mu^{4} x^{4}+737280 \mu^{4} x^{3}+27648 \mu^{4} x^{2} \\
& -262144 \mu^{2} x^{6}-786432 \mu^{2} x^{5}-86016 \mu^{2} x^{4}-36864 \mu^{2} x^{3}-1728 \mu^{2} x^{2} \\
& -49152 \mu^{4} x y^{2}+524288 \mu^{2} x y^{4}+24576 \mu^{2} x y^{2}-221184 \mu^{6} x+20736 \mu^{4} x \\
& +589824 \mu^{6} y^{2}+688128 \mu^{4} y^{4}+9216 \mu^{4} y^{2}+262144 \mu^{2} y^{6}+12288 \mu^{2} y^{4} \\
& -2880 \mu^{2} y^{2}+165888 \mu^{8}-1944 \mu^{4}+81 \mu^{2}+786432 x^{3} y^{4}+65536 x^{2} y^{4} \\
& +786432 x^{5} y^{2}+131072 x^{4} y^{2}+40960 x^{3} y^{2}+10240 x^{2} y^{2}+262144 x^{7} \\
& +65536 x^{6}+12288 x^{5}+6144 x^{4}-576 x^{3}+144 x^{2}+262144 x y^{6}+28672 x y^{4} \\
& \left.-1344 x y^{2}-27 x\right) .
\end{aligned}
$$

The second factor on the rhs gives a circle on the $x+i y=\frac{u}{\Lambda_{2}^{2}}$ plane with radius $\left|\mu^{2}-\frac{1}{4}\right|$ and center $(x, y)=\left(\mu^{2}+\frac{1}{8}, 0\right)$. By tuning the mass $\mu$ from 0 to $\infty$, one passes through the AD point $\mu=\frac{1}{2}$ where the radius of the circle shrinks to 0 . For this mass, three regions defined through $T_{(m, m)}=0$ collapse to a point $x+i y=\frac{u_{\mathrm{AD}}}{\Lambda_{2}^{2}}=\frac{3}{8}$, which is the only root over $\mathbb{R}^{2}$ of the quadratic polynomial. This gives further evidence that the domain (5.7) is in fact correct for all $\mu \in(0, \infty) \backslash\left\{\frac{1}{2}\right\}$.

We can find the truncations of the zero locus of (5.15) that gives the partitioning (2.35) in the following way. The locus $y=0$ cannot be contained fully in $\mathcal{T}_{m}$, since otherwise the partition of $\mathcal{B}_{2}$ would be into more than six parts. By direct computation one can show that for $0<\frac{m}{\Lambda_{2}}<\frac{1}{4}$ we have $\mathcal{J}(u, \boldsymbol{m}) \leq 12^{3}$ for $u_{-}<u<u_{+}$ [recall that $\mathcal{J}(u, \boldsymbol{m})$ diverges for all $u$ approaching a singularity]. This proves that the line from $u_{-}$to $u_{+}$is contained in $\mathcal{T}_{m}$. It allows us to identify the boundary pieces $\alpha_{j} \partial \mathcal{F}$ on $\mathbb{U}$ with the boundary pieces $\partial\left(u\left(\alpha_{j} \mathcal{F}\right)\right)$ on $\mathcal{B}_{2}$, which is depicted in Fig. 13.

\section{B. Two distinct masses}

In the generic case, the two masses are distinct. As in $N_{f}=1$, we can expand and invert the $\mathcal{J}$ invariant for large $u$ to find the series $\left(\mu_{i}=\frac{m_{i}}{\Lambda_{2}}\right)$

$$
\begin{aligned}
\frac{u(\tau)}{\Lambda_{2}^{2}}= & -\frac{1}{64} q^{-\frac{1}{2}}-\frac{1}{2}\left(\mu_{1}^{2}+\mu_{2}^{2}\right) \\
& +\left(24\left(\mu_{1}^{4}+\mu_{2}^{4}\right)+16 \mu_{1}^{2} \mu_{2}^{2}-32 \mu_{1} \mu_{2}-\frac{5}{16}\right) q^{\frac{1}{2}} \\
& -128\left(\mu_{1}^{2}+\mu_{2}^{2}\right)\left(16\left(\mu_{1}^{4}+\mu_{2}^{4}\right)-14 \mu_{1} \mu_{2}+1\right) q \\
& +\mathcal{O}\left(q^{\frac{3}{2}}\right) .
\end{aligned}
$$

The double singularity $u_{*}$ in the equal mass case now splits into two distinct singularities, $u_{*}^{ \pm}$. Due to the locus of masses giving rise to $u$ planes with $\mathrm{AD}$ points, it is difficult to give a fundamental domain $\mathcal{F}_{2}(\boldsymbol{m})$ for any choice of $\boldsymbol{m}=\left(m_{1}, m_{2}\right)$. From Eq. (2.28) it is clear that there are two distinct branch points in $\mathcal{B}_{2}$. When both $m_{1}$ and $m_{2}$ are real and small, i.e. have not made a phase transition compared to $\boldsymbol{m}=0$, one branch point $u_{\mathrm{bp}, 1}$ belongs to $\mathcal{T}_{\boldsymbol{m}}$, while the other $u_{\mathrm{bp}, 2}$ does not. However, $\mathcal{J}\left(u_{\mathrm{bp}, 2}\right)=j\left(\tau_{\mathrm{bp}, 2}\right) \in \mathbb{R}$ is also real but larger than $12^{3}$. A natural choice of branch cuts is along the tessellation $\{\tau \in \mathbb{H} \mid j(\tau) \in \mathbb{R}\}$, which aside from (2.38) contains the $\operatorname{SL}(2, \mathbb{Z})$ images of the positive imaginary axis. The plot of the partitioning $\mathcal{T}_{m}$ shows a feature found already in $N_{f}=1$ with a complex mass (see Sec. IV D): the $u$ plane is partitioned into only five regions, which is due to two regions $u\left(\alpha_{j} \mathcal{F}\right)$ being glued along pairs of branch cuts (see Fig. 14). The splitting of $u_{*}$ into two distinct singularities in this case does not require the two regions $T S \mathcal{F}$ and $T S T^{-1} \mathcal{F}$ to taper to distinct cusps, as we have that both $T S, T S T^{-1}: i \infty \mapsto 1$. The two singularities are rather split due to the branch cut, and the limit of $u(\tau)$ as $\tau \rightarrow 1$ depends on the path from which $\tau=1$ is approached. This is different from $u_{+} \neq u_{-}$, where the boundary pieces near the cusps are not identified.

\section{The massless theory}

When we go to the massless theory we now find

$$
\begin{aligned}
\frac{u(\tau)}{\Lambda_{2}^{2}} & =-\frac{1}{8} \frac{\vartheta_{3}(\tau)^{4}+\vartheta_{4}(\tau)^{4}}{\vartheta_{2}(\tau)^{4}}=-\frac{1}{8}-\frac{1}{64}\left(\frac{\eta\left(\frac{\tau}{2}\right)}{\eta(2 \tau)}\right)^{8}, \\
& =-\frac{1}{64}\left(q^{-1 / 2}+20 q^{1 / 2}-62 q^{3 / 2}+216 q^{5 / 2}+\mathcal{O}\left(q^{7 / 2}\right)\right) .
\end{aligned}
$$

This function is the completely replicable function of class $4 \mathrm{C}$ and is a Hauptmodul for $\Gamma(2)$ [86-88]. The physical discriminant becomes $\Delta=\left(u+\frac{\Lambda_{2}^{2}}{8}\right)^{2}\left(u-\frac{\Lambda_{2}^{2}}{8}\right)^{2}$. The two cusps correspond to $u(0)=-\frac{\Lambda_{2}^{2}}{8}$ and $u(1)=+\frac{\Lambda_{2}^{2}}{8}$. They are associated with the particles of charges $(1,0)$ and $(1,1)$ becoming massless. 

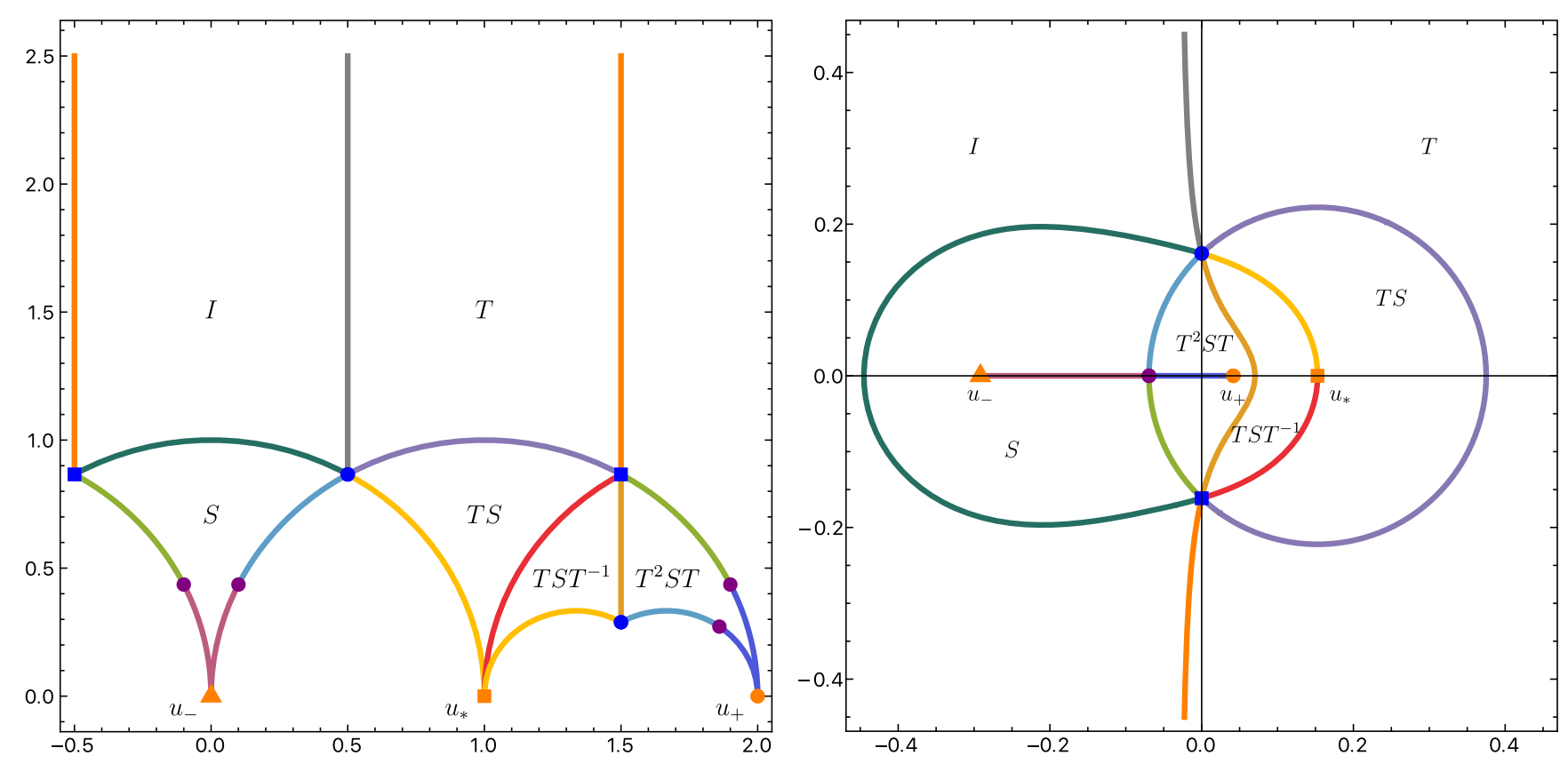

FIG. 13. Identification of the components of the partitioning $\mathcal{T}_{(m, m)}$ in $N_{f}=2$ for the particular choice $m=\frac{\Lambda_{2}}{6}$. The $u$-plane $\mathcal{B}_{2}$ is partitioned into six regions $u(\alpha \mathcal{F})$, with the $\alpha \in \operatorname{SL}(2, \mathbb{Z})$ given in both pictures. The branch point (purple) identifies four points on $\partial \mathcal{F}(m, m)$. A natural choice of branch cut is on the circle around $\tau=1$ with radius 1, as suggested in Fig. 12 (we omit it in this figure for readability). The singularities $u_{ \pm}$correspond to a single massless particle each and thus lie in the interior of a $u(\alpha \mathcal{F})$. The singularity $u_{*}$ is double and thus lies on the boundary of two such regions. The boundary pieces of $\mathcal{F}(m, m)$ are pairwise identified, which can be found by comparing $\mathcal{F}(m, m)$ with the curve $T_{(m, m)}=0$. Gluing the corresponding boundary pieces results in a Riemann surface of genus 0 with punctures.
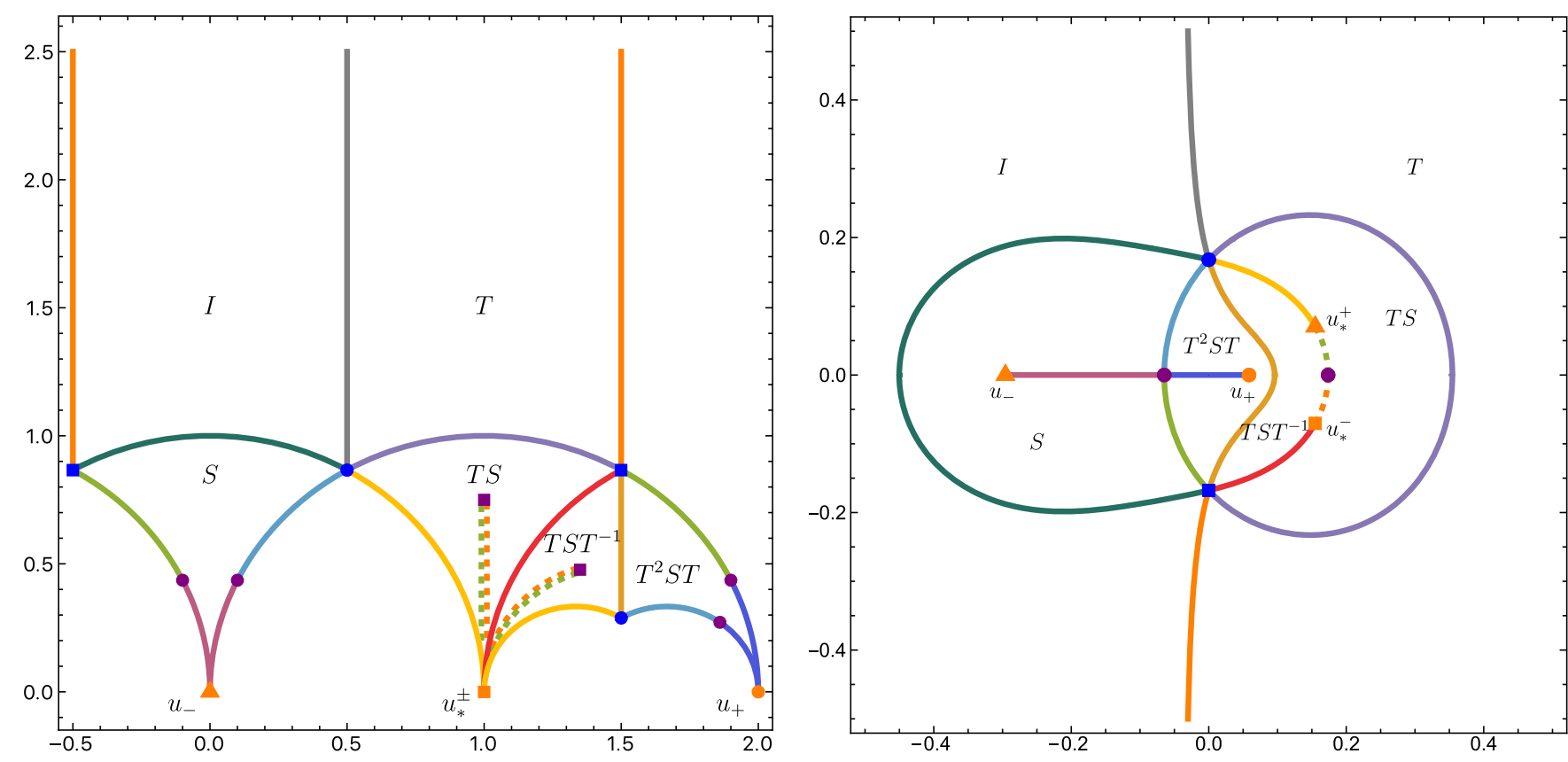

FIG. 14. Identification of the components of the partitioning $\mathcal{T}_{\left(m_{1}, m_{2}\right)}$ in $N_{f}=2$ for the particular choice $\mu_{1}=\frac{1}{10}$ and $\mu_{2}=\frac{1}{4}$. The $u$ plane $\mathcal{B}_{2}$ is naïvely partitioned into six regions $u(\alpha \mathcal{F})$, with the $\alpha \in \operatorname{SL}(2, \mathbb{Z})$ given in both pictures. Two regions $u(T S \mathcal{F})$ and $u\left(T S T^{-1} \mathcal{F}\right)$ are however glued along the pairs of branch cuts (dotted), running from the two singular points $u_{*}^{ \pm}$(orange, square) to the branch point $\tau_{\mathrm{bp}, 2}$ (purple, square). They do not belong to the partitioning $\mathcal{T}_{\boldsymbol{m}}$. A natural choice for the branch cut is along the lines where $j(\tau)$ is real. 

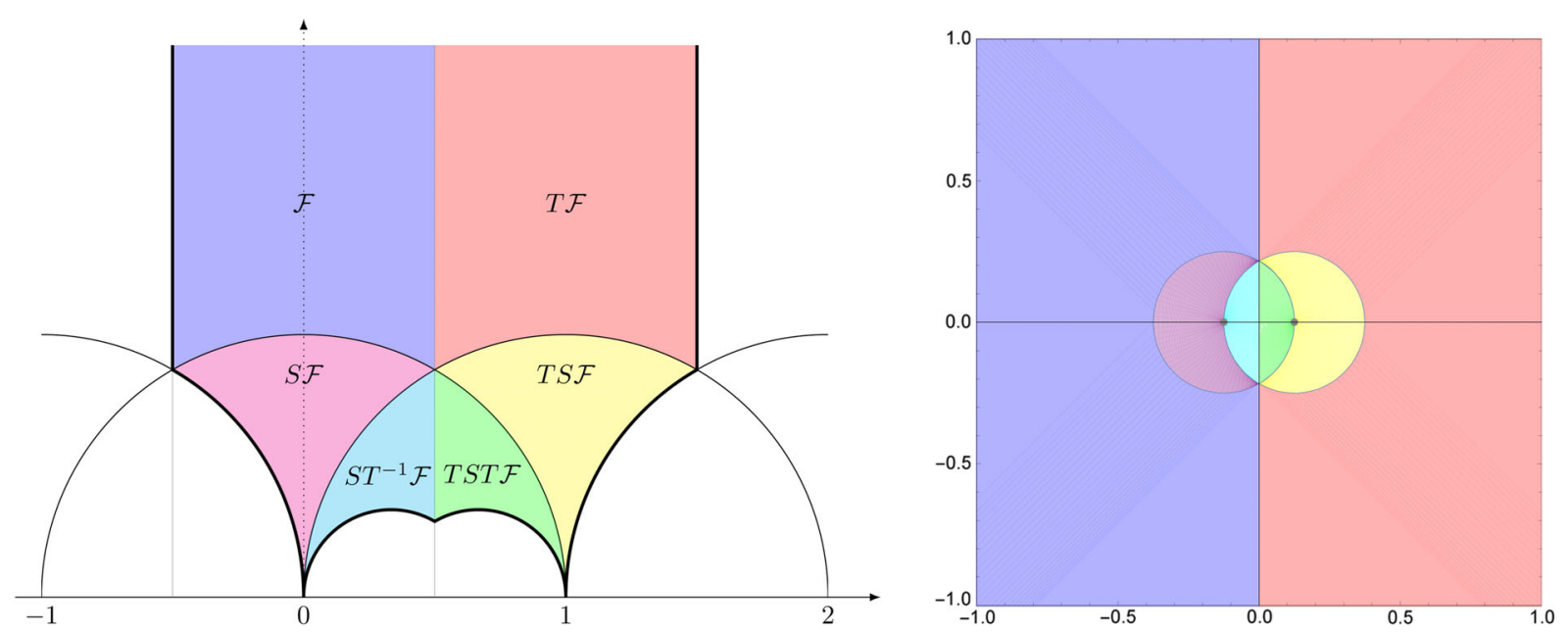

FIG. 15. Left: fundamental domain of $\Gamma(2)$. This is the duality group of massless $N_{f}=2$. All three cusps $\{i \infty, 0,1\}$ have width 2 . Right: plot of the massless $N_{f}=2 u$-plane as the union of the images of $u$ under the ind $\Gamma(2)=6 \operatorname{SL}(2, \mathbb{Z})$ images of $\mathcal{F}$. Here, we use the decomposition $\Gamma(2) \backslash \mathbb{W}=\bigcup_{k, \ell=0}^{1} T^{\ell} S^{k} \mathcal{F} \cup S T^{-1} \mathcal{F} \cup T S T \mathcal{F}$. There is a $\mathbb{Z}_{2}$ symmetry which acts by $u \mapsto-u$. The singularities $\tau=0,1$ are both touched by two triangles each.

A fundamental domain for $\Gamma(2)$ is given by

$\mathcal{F}_{2}(0,0)=\mathcal{F} \cup T \mathcal{F} \cup S \mathcal{F} \cup T S \mathcal{F} \cup S T^{-1} \mathcal{F} \cup T S T \mathcal{F}$

and is plotted in Fig. 15 together with the map to the $u$ plane. This picture gives rise to the dessin d'enfant of the $j$ invariant [[89], Fig. 6], as $u$ is a linear function of the modular $\lambda$ invariant, which has critical points $\lambda=0,1, \infty$.

\section{Type II I AD mass}

If we choose $m_{1}=m_{2}=m_{\mathrm{AD}}=\frac{1}{2} \Lambda_{2}$, then we find a $u$ plane with an $\mathrm{AD}$ theory of type III located at $u=u_{\mathrm{AD}}=$ $\frac{3}{8} \Lambda_{2}^{2}$ [34]. Three singularities collide in this point, while one remains at $u_{0}=-\frac{5}{8} \Lambda_{2}^{2}$. The discriminant now takes the form

$$
\Delta=\left(u-u_{\mathrm{AD}}\right)^{3}\left(u-u_{0}\right)
$$

Using $\Gamma(2)$ as an intermediate field of the sextic equation, we can show that

$$
\begin{aligned}
\frac{u(\tau)}{\Lambda_{2}^{2}} & =-\frac{f_{2 \mathrm{~B}}\left(\frac{\tau}{2}\right)+40}{64} \\
& =-\frac{1}{64}\left(q^{-1 / 2}+16+276 q^{1 / 2}-2048 q+\mathcal{O}\left(q^{3 / 2}\right)\right),
\end{aligned}
$$

where $f_{2 \mathrm{~B}}$ was defined in Eq. (5.9), and it is the McKayThompson series of class 2B [86-88]. It is a Hauptmodul for $\Gamma_{0}(2)$. Therefore, $u$ is a modular function for $\Gamma^{0}(2)$. A fundamental domain of $\Gamma^{0}(2)$ is

$$
\mathcal{F}_{2}\left(\boldsymbol{m}_{\mathrm{AD}}\right)=\mathcal{F} \cup T \mathcal{F} \cup S \mathcal{F}
$$

and is shown in Fig. 16. It has index 3 in $\operatorname{PSL}(2, \mathbb{Z})$, since three mutually nonlocal singularities have collided. This can also be seen from the fact that the curve reads

$$
j(\tau)=\frac{\left(f_{2 \mathrm{~B}}\left(\frac{\tau}{2}\right)+16\right)^{3}}{f_{2 \mathrm{~B}}\left(\frac{\tau}{2}\right)} .
$$

One has that $u\left(\tau_{\mathrm{AD}}\right)=u_{\mathrm{AD}}$ whenever $f_{2 \mathrm{~B}}\left(\frac{\tau_{\mathrm{AD}}}{2}\right)=-64$, whose solution locus intersects with our choice of $\Gamma^{0}(2) \backslash \mathbb{M}$ in $\tau_{\mathrm{AD}}=1+i$. This can be proven from the $S$ transformation of the Dedekind $\eta$ function. It is also easy to check that $u(0)=u_{0}$. Taking the proper limits in Eq. (5.4)

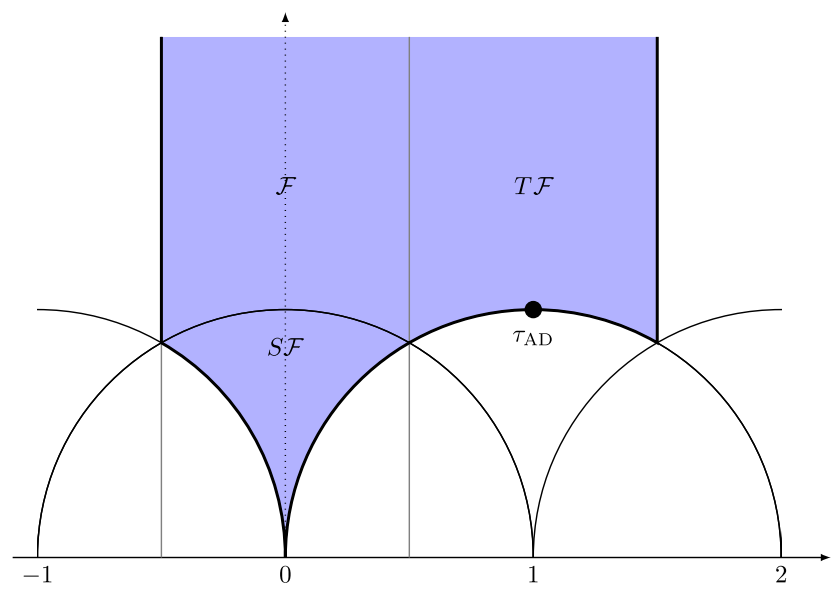

FIG. 16. Fundamental domain of $\Gamma^{0}(2)$. This is the duality group of $N_{f}=2$ with masses $\boldsymbol{m}=\frac{1}{2}\left(\Lambda_{2}, \Lambda_{2}\right)$. The AD point corresponds to the elliptic fixed point $\tau_{\mathrm{AD}}=1+i$. 
we directly find $\frac{d u}{d \tau}$ as well as $\frac{d a}{d u}$ and we can check that they satisfy the Matone relation

$$
\frac{d u}{d \tau}=-\frac{16 \pi i}{2} \hat{\Delta}\left(\frac{d a}{d u}\right)^{2},
$$

which is consistent with Eq. (3.13). Both branch points of the $N_{f}=2$ theory have collided along with the singularities where mutually nonlocal states become massless. The monodromies are

$$
\begin{gathered}
M_{0}=S T S^{-1}=\left(\begin{array}{cc}
1 & 0 \\
-1 & 1
\end{array}\right), \\
M_{\mathrm{AD}}=T S^{-1} T^{-1}=\left(\begin{array}{ll}
-1 & 2 \\
-1 & 1
\end{array}\right),
\end{gathered}
$$

and they satisfy $M_{0} M_{\mathrm{AD}}=M_{\infty}$ with $M_{\infty}=P T^{-2}$. Furthermore, $M_{\mathrm{AD}}^{4}=\mathbb{1}$, such that $\tau_{\mathrm{AD}}$ indeed is an elliptic fixed point of $\Gamma^{0}(2)$. The $\mathrm{AD}$ monodromy is conjugate to $S^{-1}$, which fixes $\tau=i$. Since $\tau_{\mathrm{AD}}=i+1$, this gives a path in $\tau$ space.

\section{THE $N_{f}=3$ CURVE}

We will start by discussing the $N_{f}=3$ theory with one nonzero mass, $\boldsymbol{m}=(m, 0,0)$, where we can find an explicit expression for $u$ in terms of Jacobi theta functions. After this we discuss the generic mass case, the massless theory and a number of theories with specific AD masses.

\section{A. One nonzero mass}

For the general theory it turns out to be complicated to find closed expressions for $u$, but if we only keep one nonzero mass, $\boldsymbol{m}=(m, 0,0)$, we can make more progress. Four of the strong coupling singularities now merge in pairs of two and the physical discriminant becomes

$$
\Delta=\left(u-u_{+}\right)^{2}\left(u-u_{-}\right)^{2}\left(u-u_{*}\right)
$$

with

$$
u_{ \pm}= \pm \frac{m \Lambda_{3}}{8}, \quad u_{*}=\frac{\Lambda_{3}^{2}}{2^{8}}+m^{2}
$$

There are two $\mathrm{AD}$ points at $m=m_{\mathrm{AD}}= \pm \frac{1}{16} \Lambda_{3}$ and $u=$ $u_{\mathrm{AD}}=\frac{1}{128} \Lambda_{3}^{2}$ where either $u_{+}$or $u_{-}$merges with $u_{*}$ to give a type III singular fiber. We now find that the sextic equation for $u$ again splits over the intermediate field $\Gamma(2)$. In this case there is only one solution that has $|u| \rightarrow \infty$ for $\tau \rightarrow i \infty$, and as has been mentioned before this has $u \rightarrow-\infty$. This is then the reason why we have persistently chosen this convention in all other cases, to make the decoupling limits from $N_{f}=3$ consistent. We find that

$$
\begin{aligned}
\frac{u}{\Lambda_{3}^{2}}= & -\frac{2 \vartheta_{3}^{4} \vartheta_{4}^{4}+\left(\vartheta_{3}^{4}+\vartheta_{4}^{4}\right) \sqrt{f_{3}}}{64 \vartheta_{2}^{8}}, \\
= & -\frac{1}{2^{12}}\left(\frac{1}{q}+\left(-8+4096 \mu^{2}\right)+4\left(5+32768 \mu^{2}\right.\right. \\
& \left.\left.-4194304 \mu^{4}\right) q+\mathcal{O}\left(q^{2}\right)\right)
\end{aligned}
$$

where we have defined $f_{3}=\frac{64 m^{2}}{\Lambda_{3}^{2}} \vartheta_{2}^{8}+\vartheta_{3}^{4} \vartheta_{4}^{4}$ and $\mu=\frac{m}{\Lambda_{3}}$. It is straightforward to calculate the other interesting quantities explicitly from Eq. (6.3) and to check that the generalized Matone relation is satisfied also in this case.

Similarly to what we saw in the equal mass $N_{f}=2$ case, the square roots will introduce branch points in the moduli space. They are given by

$$
j^{\mathrm{bp}}(m)=\frac{\left(\Lambda_{3}-8 m\right)^{3}\left(\Lambda_{3}+8 m\right)^{3}}{16 m^{4} \Lambda_{3}^{2}} .
$$

By plugging in the expression for $\mathcal{J}$ in terms of $u$ we find that the branch point lies at $u_{\mathrm{bp}}=2 \mathrm{~m}^{2}$ in the $u$ plane, as is also found by studying the Matone polynomial (3.15). We can also use known relations between the $j$ invariant and theta functions to check that (6.4) coincides with $f_{3}=0$, such that the branch point of $u$ is that of the square root in (6.3).

\section{Fundamental domain}

We can repeat the method developed in $N_{f}=2$ with $\boldsymbol{m}=(m, m)$ and write down all the cusp expansions. They can be canonically normalized to match the form of the expansion at $\infty$. This allows us to find the maps $\alpha_{j} \in \mathrm{SL}(2, \mathbb{Z})$, which give the fundamental domain

$$
\begin{aligned}
& \mathcal{F}_{3}(m, 0,0) \\
& \quad=\mathcal{F} \cup S \mathcal{F} \cup S T^{-1} \mathcal{F} \cup T S T \mathcal{F} \cup T S T^{2} \mathcal{F} \cup T S T^{2} S \mathcal{F},
\end{aligned}
$$

shown in Fig. 17. It is valid for all masses $m$ that do not allow the square root to resolve. We prove below that this does not happen unless $m=0$ or $m=m_{\mathrm{AD}}=\frac{1}{16} \Lambda_{3}$.

Let us also study the paths of branch points in the fundamental domain. Similarly as in massive $N_{f}=$ 1,2 , we analyze the critical values of (6.4). We have that $\lim _{m \searrow 0} j^{\mathrm{bp}}(m)=+\infty, j^{\mathrm{bp}}\left(m_{\mathrm{AD}}\right)=12^{3}, j^{\mathrm{bp}}\left(\frac{\Lambda_{3}}{8}\right)=0$ and $\lim _{m \rightarrow \infty} j^{\mathrm{bp}}(m)=-\infty$. It is easy to show that $j^{\mathrm{bp}}:(0, \infty) \rightarrow \mathbb{R}$ is monotonically decreasing and therefore injective.

Since $u_{\mathrm{bp}}=2 m^{2}$, we have that at $m=0$ the branch points coincides with $u_{+}$and $u_{-}$. At the AD point, $m=\frac{\Lambda_{3}}{16}$, it collides along with $u_{*}$ and $u_{+}$. Finally, for $m \rightarrow \infty$ it diverges, just as $u_{*}$ does. This fixes the points $\tau=0$ and $\tau=1$ for $m=0, \tau=\tau_{\mathrm{AD}}=\frac{1}{2}+\frac{i}{2}$ for $m=m_{\mathrm{AD}}$ and $\tau=\frac{1}{2}$ 


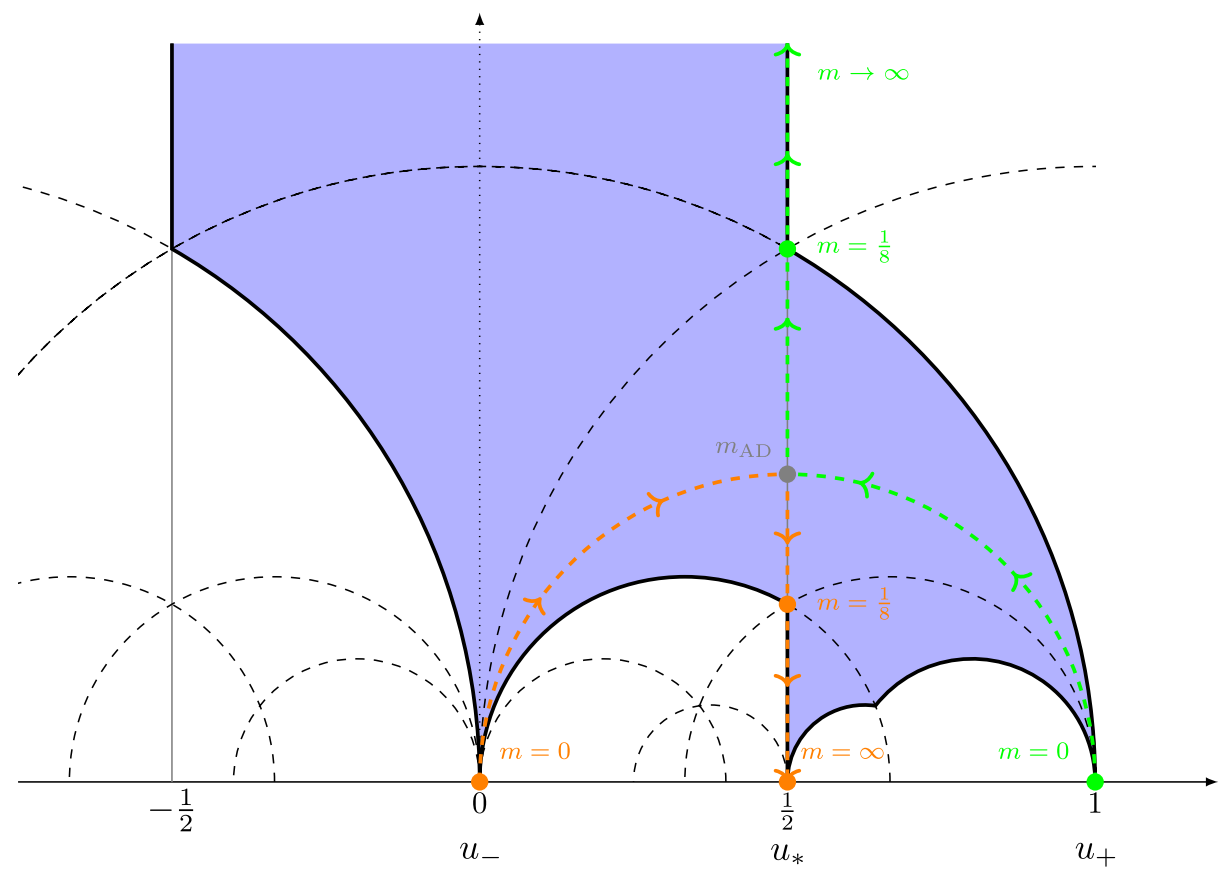

FIG. 17. Fundamental domain for $N_{f}=3$ with $\boldsymbol{m}=(m, 0,0)$. The dashed lines corresponds to the path of the branch points on the positive ray from massless to infinite mass.

or $i \infty$ for $m=\infty$. The simplest curves connecting these three points are quarter circles with radius $\frac{1}{2}$ around $\tau=\frac{1}{2}$ starting from either $\tau=0$ or $\tau=1$, followed by a vertical path from $\frac{1}{2}+\frac{i}{2}$ to either $\frac{1}{2}$ or $i \infty$.

The fundamental domain together with the path of the branch points found from the above considerations is shown in Fig. 17.

The various checks of the branch points paths are analogous to $N_{f}=1,2$. We can plot $j(\tau)$ along these curves and find that it has the same global properties and critical points as (6.4). The intermediate value $j(\tau)=0$ corresponds to $m=\frac{\Lambda_{3}}{8}$ and $\tau=\frac{1}{2}+\frac{1}{2 \sqrt{2}} i$, which is in the $\operatorname{SL}(2, \mathbb{Z})$ orbit of $\omega_{3}$. Along the branch point locus, $u$ simplifies to

$$
u\left(\tau_{\mathrm{bp}}\right)=-\frac{f_{2 \mathrm{~B}}\left(\tau_{\mathrm{bp}}\right)}{2^{13}},
$$

with $f_{2 \mathrm{~B}}$ given by Eq. (5.9). On the paths in Fig. 17 this function behaves precisely as $u_{\mathrm{bp}}(m)=2 m^{2}$.

\section{Limits to zero, $A D$ and infinite mass}

As in the $\boldsymbol{m}=(m, m) N_{f}=2$ theory, there are three interesting limits: $m \rightarrow 0, m \rightarrow \infty$ and $m \rightarrow m_{\mathrm{AD}}$. In the massless limit, we aim to recover $\Gamma_{0}(4) \backslash \mathbb{H}$. This is not difficult to see: under $\Gamma_{0}(4)$, we can identify $T S T^{2} S \mathcal{F}$ with $S T^{-2} S \mathcal{F}$, and similarly $T S T^{2} \mathcal{F}$ with $S T^{-2} \mathcal{F}$, since the transition maps are in $\Gamma_{0}(4)$. This gives precisely Fig. 19.
By decoupling the massive hypermultiplet, the theory flows to massless $N_{f}=2$. We find that $u_{*} \rightarrow \infty$, while $u_{ \pm} \rightarrow \pm \frac{\Lambda_{2}^{2}}{8}$. From Sec. V C it is clear that the singularities $u_{ \pm}$do not move in $\tau$ space. The cusp region $T S T^{2} S \mathcal{F}$ is identified with $T \mathcal{F}$ under the duality group $\Gamma(2)$ of massless $N_{f}=2$. Moreover, the remaining differing triangle $T S T^{2} \mathcal{F}$ can be mapped to $T S \mathcal{F}$ using $\Gamma(2)$. This then gives precisely $\Gamma(2) \backslash \mathbb{W}$ as in Fig. 15 .

Finally, in the limit $m \rightarrow m_{\mathrm{AD}}=\frac{1}{16} \Lambda_{3}$ the singularities $u_{*}$ and $u_{+}$collide. Since they drop out of the curve, we should remove all regions near those cusps. In Fig. 17 we can remove the triangles $T S T \mathcal{F}, T S T^{2} \mathcal{F}$ and $T S T^{2} S \mathcal{F}$, after which the index 3 group $\Gamma_{0}(2)$ remains. This is precisely what is found as the duality group of the $\boldsymbol{m}=\left(m_{\mathrm{AD}}, 0,0\right)$ theory, as shown in Fig. 16. The preimage of the merged nonlocal singularities $u_{\mathrm{AD}}$ is the point $\tau_{\mathrm{AD}}$, which lies in the interior of $\llbracket$ and corresponds to the point where the branch points have collided.

\section{B. Generic masses}

For generic masses $\boldsymbol{m}=\left(m_{1}, m_{2}, m_{3}\right)$, the order parameter reads

$$
\begin{aligned}
\frac{u}{\Lambda_{3}^{2}}= & -\frac{1}{2^{12}}\left(\frac{1}{q}+\left(-8+4096 M_{2}\right)+4\left(5+32768 M_{2}\right.\right. \\
& +3670016 M_{3}-4194304 M_{4} \\
& \left.\left.-4194304 M_{4}^{\prime}\right) q+\mathcal{O}\left(q^{2}\right)\right),
\end{aligned}
$$



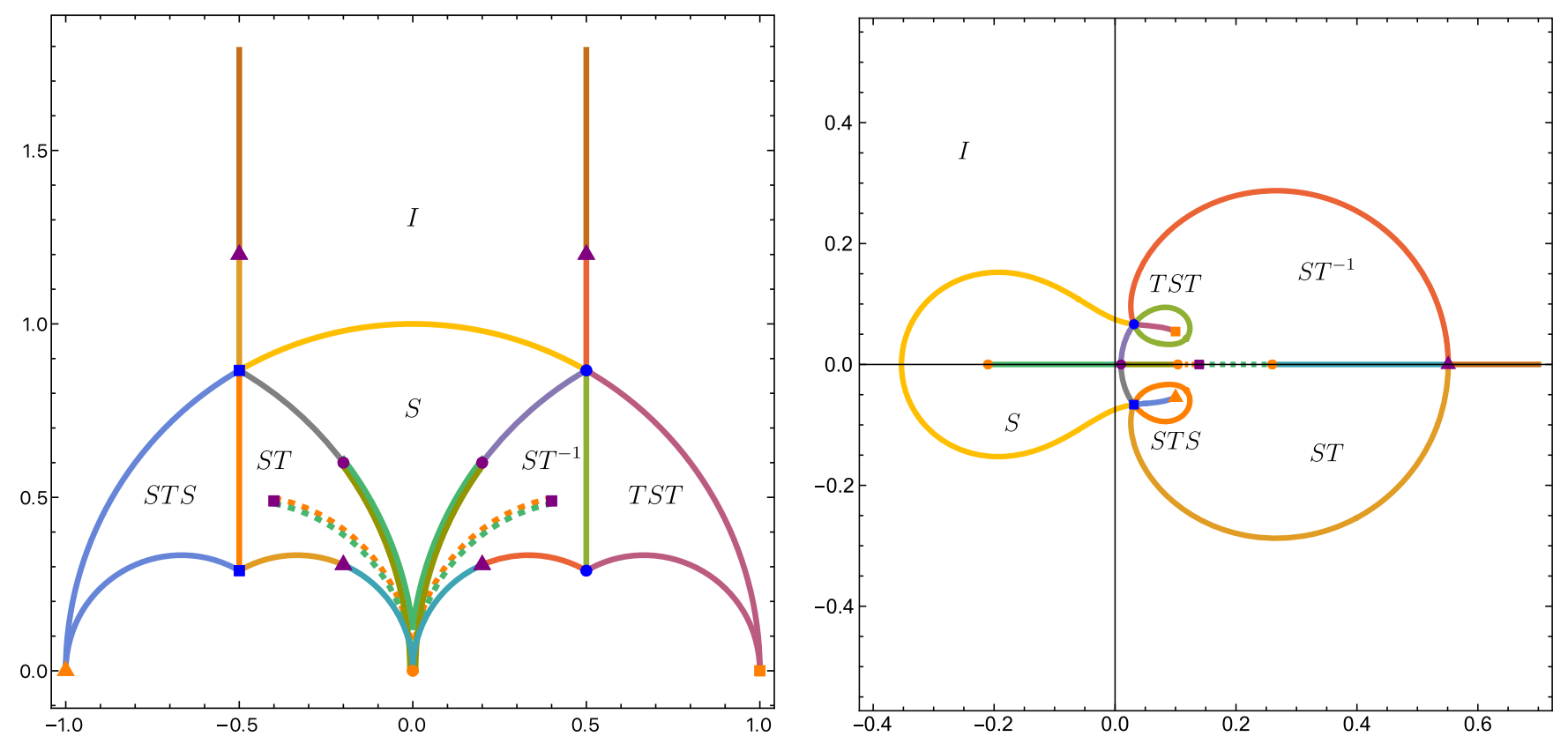

FIG. 18. Identification of the components of the partitioning $\mathcal{T}_{\left(m_{1}, m_{2}, m_{3}\right)}$ in $N_{f}=3$ for the particular choice $\mu_{1}=\frac{1}{10}, \mu_{2}=\frac{3}{10}$ and $\mu_{3}=\frac{5}{10}$. The $u$-plane $\mathcal{B}_{3}$ is partitioned into five regions $u(\alpha \mathcal{F})$, as those for $S T^{-1}$ and $S T$ are glued by two branch cuts. The fundamental domain is given by six copies of $\mathcal{F}$, with three pairs of branch points (purple). Two branch points (triangle and disk) lie on $\mathcal{T}_{3}$. The third (square) lies in the interior and glues the copies $S T \mathcal{F}$ and $S T^{-1} \mathcal{F}$. A natural choice for the branch cuts notlying in $\mathcal{T}_{3}$ (dashed) is on the real axis in the $u$ plane, for which the path in $\mathbb{H}$ is along the tessellation $\operatorname{SL}(2, \mathbb{Z}) \cdot i \mathbb{R}_{>0}$. The singularity at $\tau=0$ does not have width 3 as apparent, since due to the branch cuts $u(\tau)$ assumes three different values depending on which side of the branch cut $\tau=0$ is approached from.

where the coefficients $M_{i}$ are the symmetric polynomials defined in Eq. (3.16) for the variables $\frac{m_{i}}{\Lambda_{3}}$. There are five generally distinct singular points.

Due to the $N_{f}=3$ distinct branch points on the $u$ plane, the fundamental domain for a given mass $\boldsymbol{m}$ has an intricate web of branch cuts. Furthermore, the fundamental domains $\mathcal{F}_{3}(\boldsymbol{m})$ change as $\boldsymbol{m}$ passes through $\mathcal{L}_{3}^{\mathrm{AD}}$ (see Fig. 3). A fundamental domain $\mathcal{F}_{3}(\boldsymbol{m})$ can also change when $\boldsymbol{m}$ is varied such that $\Delta_{3}$ has any double root, and when branch points in $\mathbb{U}$ pass through the $\operatorname{SL}(2, \mathbb{Z})$ tessellation $\mathcal{T}_{\mathbb{H}}(2.38)$.

For any given mass $\boldsymbol{m}$ one easily computes $T_{3}$ from Eq. (2.36), and truncates the plot of the level set to the region where $\mathcal{J}\left(u, \boldsymbol{m}, \Lambda_{3}\right) \leq 12^{3}$. The branch points $u_{\mathrm{bp}}$ are the zeros of $P_{3}^{\mathrm{M}}$ (3.15). On the upper half-plane $\mathbb{H}$, a branch point $\tau_{\mathrm{bp}}$ is any of the $\operatorname{SL}(2, \mathbb{Z})$ images of $\left(\left.j\right|_{\mathcal{F}}\right)^{-1}\left(\mathcal{J}\left(u_{\mathrm{bp}}\right)\right)$. When $\mathcal{J}\left(u_{\mathrm{bp}}\right) \leq 12^{3}$, then obviously $\tau_{\mathrm{bp}} \in \mathcal{T}_{\mathbb{H}}$. If $\mathcal{J}\left(u_{\mathrm{bp}}\right)>12^{3}$, then $\tau_{\mathrm{bp}} \in \operatorname{SL}(2, \mathbb{Z}) \cdot i \mathbb{R}_{>0}$. Lastly, if $\mathcal{J}\left(u_{\mathrm{bp}}\right) \notin \mathbb{R}$, then $\tau_{\text {bp }}$ is an interior point of an $\operatorname{SL}(2, \mathbb{Z})$ copy of $\mathcal{F}$.

In Fig. 18 we plot the $u$ plane and corresponding fundamental domain for three distinct masses. The five distinct singular points are partitioned into five regions $u(\alpha \mathcal{F})$, where two of them are glued by branch cuts.

\section{The massless theory}

When sending $m \rightarrow 0$ from above we find

$$
\begin{aligned}
\frac{u(\tau)}{\Lambda^{2}} & =-\frac{1}{64} \frac{\vartheta_{3}(\tau)^{2} \vartheta_{4}(\tau)^{2}}{\left(\vartheta_{3}(\tau)^{2}-\vartheta_{4}(\tau)^{2}\right)^{2}}=-\frac{1}{2^{12}}\left(\frac{\eta(\tau)}{\eta(4 \tau)}\right)^{8}, \\
& =-\frac{1}{2^{12}}\left(q^{-1}-8+20 q-62 q^{3}+\mathcal{O}\left(q^{9 / 2}\right)\right) .
\end{aligned}
$$

It is the completely replicable function of class $4 \mathrm{C}$ and a Hauptmodul for $\Gamma_{0}(4)$ [86-88]. The physical discriminant is $\Delta=u^{4}\left(u-\frac{\Lambda_{3}^{2}}{2^{8}}\right)$, and one finds that the singularities are located at $u(0)=0$ and $u\left(\frac{1}{2}\right)=\frac{\Lambda_{3}^{2}}{2^{8}}$. At $\tau=0$ a dyon with charge $(1,0)$ becomes massless, while at $\tau=\frac{1}{2}$ one finds instead that a dyon with charge $(2,1)$ becomes massless. The massless $N_{f}=3 u$ plane has no global symmetries.

A choice of fundamental domain for $\Gamma_{0}(4)$ is

$$
\begin{aligned}
& \mathcal{F}_{3}(0,0,0) \\
& \quad=\mathcal{F} \cup S \mathcal{F} \cup S T \mathcal{F} \cup S T^{-1} \mathcal{F} \cup S T^{-2} \mathcal{F} \cup S T^{-2} S \mathcal{F},
\end{aligned}
$$

and is shown in Fig. 19. 


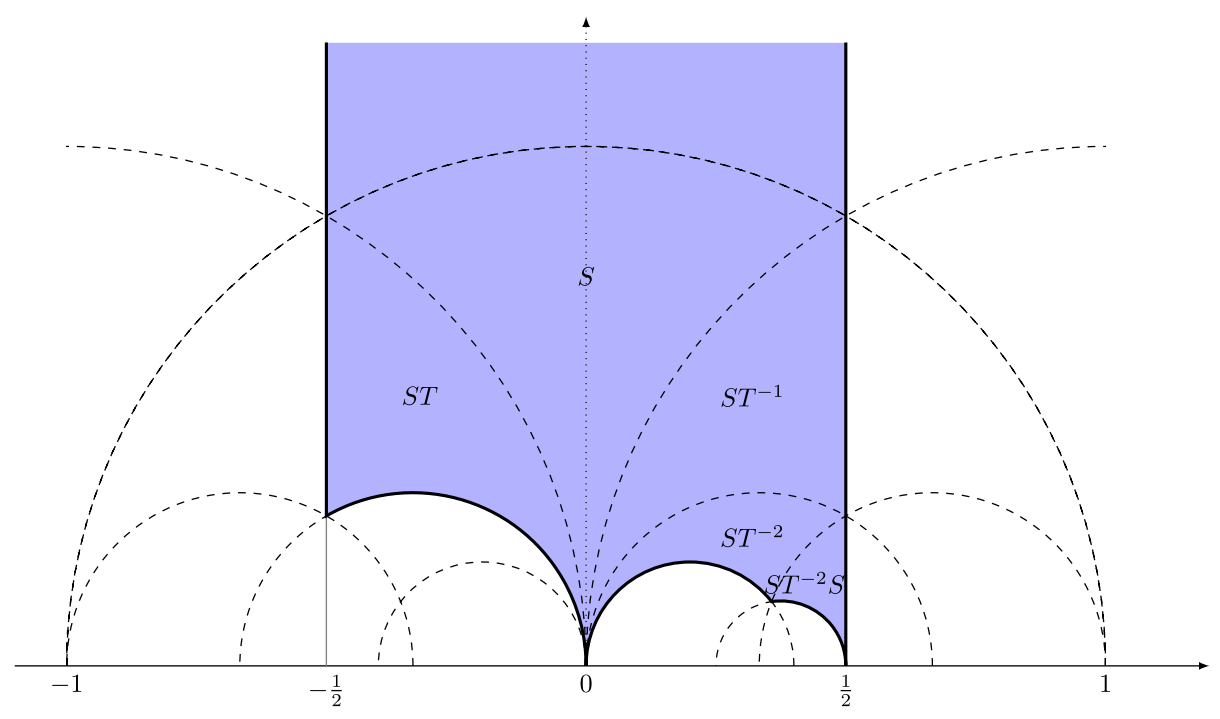

FIG. 19. Fundamental domain of $\Gamma_{0}(4)$, the duality group of massless $N_{f}=3$. The cusp at $\tau=0$ has width four, while the cusp at $\tau=\frac{1}{2}$ has width one.

\section{Type IV AD mass}

As illustrated in Fig. 3, on the generic mass $N_{f}=3 u$ plane, there is not only the IV AD point but also a variety of III and II points. We will give a few explicit examples of the $u$ plane of the theories with masses tuned to these specific values, starting with the most symmetric case.

For $\boldsymbol{m}=(m, m, m)$ and $m=\frac{1}{8} \Lambda_{3}$, four mutually nonlocal singularities collide in $u_{\mathrm{AD}}=\frac{1}{32} \Lambda_{3}^{2}$. The remaining singularity is $u_{0}=-\frac{19}{2^{8}} \Lambda_{3}^{2}$ and never collides with the other four. The physical discriminant is $\Delta=\left(u-u_{0}\right)\left(u-u_{\mathrm{AD}}\right)^{4}$. One finds that

$$
\frac{u}{\Lambda_{3}^{2}}=-\frac{j^{*}+304}{2^{12}}
$$

where

$$
\begin{aligned}
j^{*}= & 432 \frac{\sqrt{j}+\sqrt{j-1728}}{\sqrt{j}-\sqrt{j-1728}}=432 \frac{E_{4}^{\frac{3}{2}}+E_{6}}{E_{4}^{\frac{3}{2}}-E_{6}} \\
= & \frac{1}{q}-120+10260 q-901120 q^{2} \\
& +91676610 q^{3}+O\left(q^{4}\right)
\end{aligned}
$$

is the Ramanujan-Sato series of level 1 [90-92]. ${ }^{12}$

\footnotetext{
${ }^{12}$ The Ramanujan-Sato series generalize Ramanujan's formula for $\frac{1}{\pi}$ as a series of quotients of modular forms. They exist for level 1 up to 11 and beyond. The level 1 series is the only one whose generating function can not be expressed by an $\eta$ quotient [93].
}

Inverting (6.11) we find

$$
j=\frac{\left(j^{*}+432\right)^{2}}{j^{*}} .
$$

Using this and a discussion similar to the massless $N_{f}=1$ case for the transformations of $j^{*}$ we find that the singularities are located at $\left(\omega_{j}=e^{2 \pi i / j}\right)$

$u(i \infty)=\infty, \quad u\left(\omega_{3}\right)=u_{\mathrm{AD}}, \quad u(0)=u_{0}$.

We can read off from Eq. (6.12) that $\operatorname{ord}\left(g_{2}, g_{3}, \Delta\right)=$ $(2,2,4)$ at the AD point, such that according to Table III we indeed have a singular fiber of Kodaira type IV [82].

From Eq. (6.12) we read off that the duality group $\Gamma_{j^{*}}$ has index 2, which is consistent with the previous cases in $N_{f}=1,2$ in that a factor of $\left(u-u_{\mathrm{AD}}\right)^{4}$ has canceled from $g_{2}^{3}$ and $\Delta$, and therefore does not contribute to the index. The fundamental region of $u$ is therefore of index 2 with 0 , $\omega_{3}$ and $i \infty$ on its boundary. However, there is no index 2 subgroup of $\operatorname{SL}(2, \mathbb{Z})$ with two distinct cusps [[81], Table 4.1]. ${ }^{13}$ This agrees with the fact that (6.10) is not a classical modular form and the monodromy group does not promote to a modular group since its action on $u$ is not associative (see Sec. IVA). We can nevertheless propose a fundamental region

\footnotetext{
${ }^{13}$ In fact, there is exactly one index 2 subgroup of $\operatorname{SL}(2, \mathbb{Z})$ and it has only one cusp of width two. This group is sometimes referred to as $\Gamma_{0}(1)^{*}$ and is generated by $T S$ and $T^{2}$, and the Hauptmodul is given by $\sqrt{j-1728}=8 \frac{\left(\vartheta_{2}^{4}+\vartheta_{3}^{4}\right)\left(\vartheta_{3}^{4}+\vartheta_{4}^{4}\right)\left(\vartheta_{4}^{4}-\vartheta_{2}^{4}\right)}{\vartheta_{2}^{4} \vartheta_{3}^{4} \vartheta_{4}^{4}}$.
} 

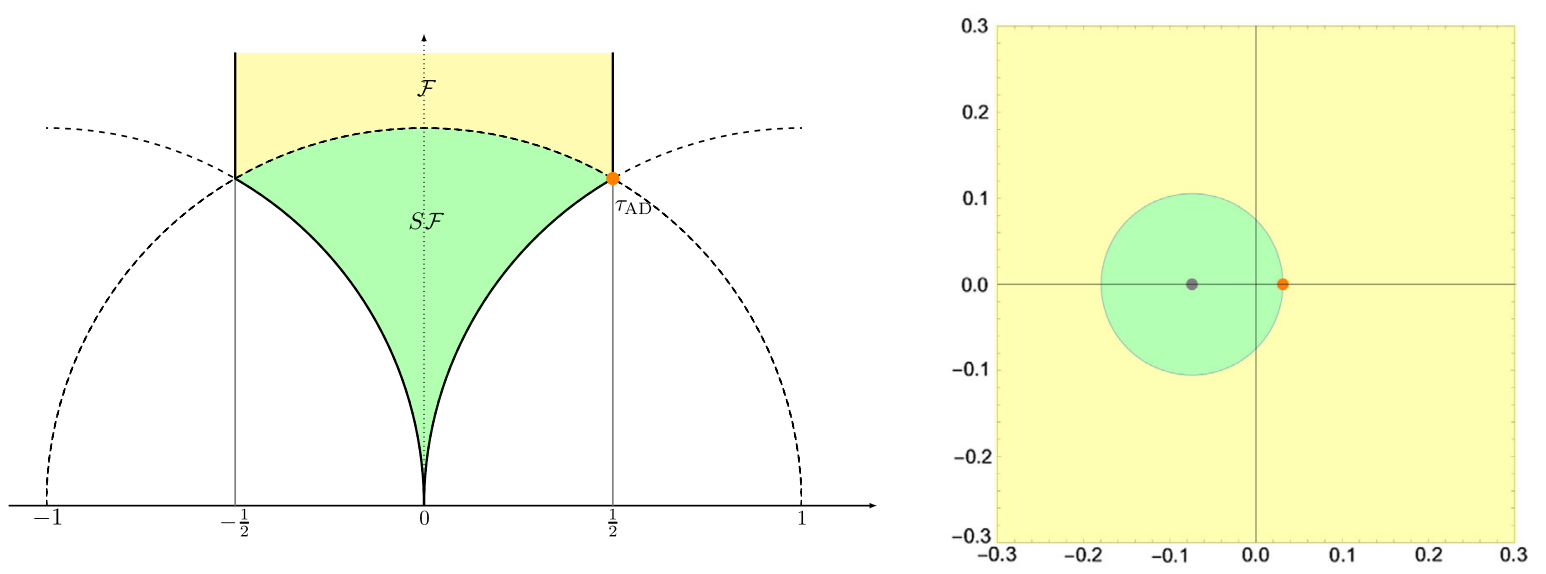

FIG. 20. Left: proposed fundamental domain of the duality group $\Gamma_{j^{*}}$ of the $N_{f}=3 \boldsymbol{m}=\frac{1}{8}\left(\Lambda_{3}, \Lambda_{3}, \Lambda_{3}\right)$ theory. It is a region with index $6-4=1+1$, elliptic fixed point $\omega_{3}$ and cusp at 0 . This uniquely fixes it. Right: plot of the $u$ plane for the corresponding theory as the union of the images of $u$ under the $6-4=2 \operatorname{SL}(2, \mathbb{Z})$ images of $\mathcal{F}$ as in the left figure. The complex plane can clearly be covered by two triangles. This demonstrates that the proposal of the left figure is indeed a fundamental domain for (6.10). The weak coupling region is covered by $u(\mathcal{F})$. The strong coupling region $u(S \mathcal{F})$ contains the singular point $u_{0}$ in its interior. The $\mathrm{AD}$ point (orange) lies on the boundary.

$$
\mathcal{F}_{3}\left(\boldsymbol{m}_{\mathrm{AD}}\right)=\mathcal{F} \cup S \mathcal{F}
$$

see Fig. 20.

The monodromies are found by consistency,

$m_{0}=S T S^{-1}=\left(\begin{array}{cc}1 & 0 \\ -1 & 1\end{array}\right), \quad m_{\mathrm{AD}}=T S^{-1}=\left(\begin{array}{cc}-1 & 1 \\ -1 & 0\end{array}\right)$,

and are unique in $\operatorname{SL}(2, \mathbb{Z}) .{ }^{14}$ They fix $\tau=0$ and $\tau_{\mathrm{AD}}=e^{\pi i / 3}=\frac{1}{2}+\frac{\sqrt{3}}{2} i$, respectively, and produce the large $u$ monodromy $m_{0} m_{\mathrm{AD}}=P T^{-1}$. Just as in the massless $N_{f}=1$ case, we note that as matrices they do not form a congruence subgroup but instead generate the whole of $\operatorname{SL}(2, \mathbb{Z})$, since $m_{\infty}=P T^{-1}$ and $T m_{0} T=S$. However, $u$ is not invariant under $S$.

\section{E. Type III AD mass}

In the single mass case $\boldsymbol{m}=(m, 0,0)$ with $m_{\mathrm{AD}}=\frac{1}{16} \Lambda_{3}$, the $N_{f}=3$ curve has an $\mathrm{AD}$ point at $u_{\mathrm{AD}}=\frac{1}{128} \Lambda_{3}^{2}$. The physical discriminant is $\Delta=\left(u-u_{\mathrm{AD}}\right)^{3}\left(u-u_{0}\right)^{2}$ with $u_{0}=-\frac{1}{128} \Lambda_{3}^{2}$, which is $-u_{\mathrm{AD}}$ by coincidence. One easily finds

$$
\frac{u(\tau)}{\Lambda_{3}^{2}}=-\frac{f_{2 \mathrm{~B}}(\tau)+32}{2^{12}},
$$

with $f_{2 \mathrm{~B}}$ defined in Eq. (5.9). This fits nicely into the description as $\operatorname{ind} \Gamma_{0}(2)=3$ is equal to $6-3=3$, being

\footnotetext{
${ }^{14}$ The overall signs are fixed in the following way. The large $u$ monodromy is $P T^{-1}$. The monodromy at $m_{0}$ is oriented such that it conjugates to $T$. This fixes the sign of $m_{\mathrm{AD}}$ from the below relation.
}

the number of mutually nonlocal singularities collided at $u_{\mathrm{AD}}$. We find that $u(\tau)=u_{0}$ is equivalent to $f_{2 \mathrm{~B}}(\tau)=0$, and one can easily show that $f_{2 \mathrm{~B}}$ vanishes at the cusp $\tau=0$. Using the $S$ transformation of $\eta$, we can show that $\tau_{\mathrm{AD}}=\frac{1}{2}+\frac{i}{2}$. In terms of the Hauptmodul, the curve reads $j=\frac{\left(f_{2 \mathrm{~B}}+256\right)^{3}}{f_{2 \mathrm{~B}}^{2}}$. This shows that the $\mathrm{AD}$ point is indeed a type III singularity. It also follows that $j\left(\tau_{\mathrm{AD}}\right)=12^{3}$ and that $\tau_{\mathrm{AD}}$ is in the $\operatorname{SL}(2, \mathbb{Z})$ orbit of $i$. The duality group $\Gamma_{0}(2)$ is generated by $g_{1}=T$ and $g_{2}=S T^{2} S$. The AD point is stabilized by $g_{1} g_{2} \in \Gamma_{0}(2)$, which makes it an elliptic fixed point. A fundamental domain for $u$ is given in Fig. 21. The effective coupling at the AD point is explained through the fact that the $N_{f}=3$ branch point collides along with the three mutually nonlocal singularities in $u_{\mathrm{AD}}$, and the two branch points on the upper half-plane as drawn in Fig. 17 collide at $\tau_{\mathrm{AD}}$ for $m=m_{\mathrm{AD}}$.

The monodromies are

$$
\begin{aligned}
M_{0} & =S T^{2} S^{-1}=\left(\begin{array}{cc}
1 & 0 \\
-2 & 1
\end{array}\right), \\
M_{\mathrm{AD}} & =(T S)^{-1} S^{-1} T S=\left(\begin{array}{ll}
-1 & 1 \\
-2 & 1
\end{array}\right) .
\end{aligned}
$$

The first one describes the path around the cusp $\tau=0$, which has width 2 . The AD monodromy is conjugate to $S^{-1}$, which fixes $\tau=i$. The path is then given by the map $(T S)^{-1}: i \mapsto \tau_{\mathrm{AD}}$. The matrices (6.17) satisfy $M_{0} M_{\mathrm{AD}}=$ $M_{\infty}$ with $M_{\infty}=P T^{-1}$ and $M_{\mathrm{AD}}^{2}=\mathbb{1}$, such that $\tau_{\mathrm{AD}}$ is indeed an elliptic fixed point for $\Gamma_{0}(2)$ of order 2 . They are also related to (5.24) by conjugation with $\operatorname{diag}(2,1)$, which induces the isomorphism between the $\Gamma_{0}(2)$ and $\Gamma^{0}(2)$ curves. 


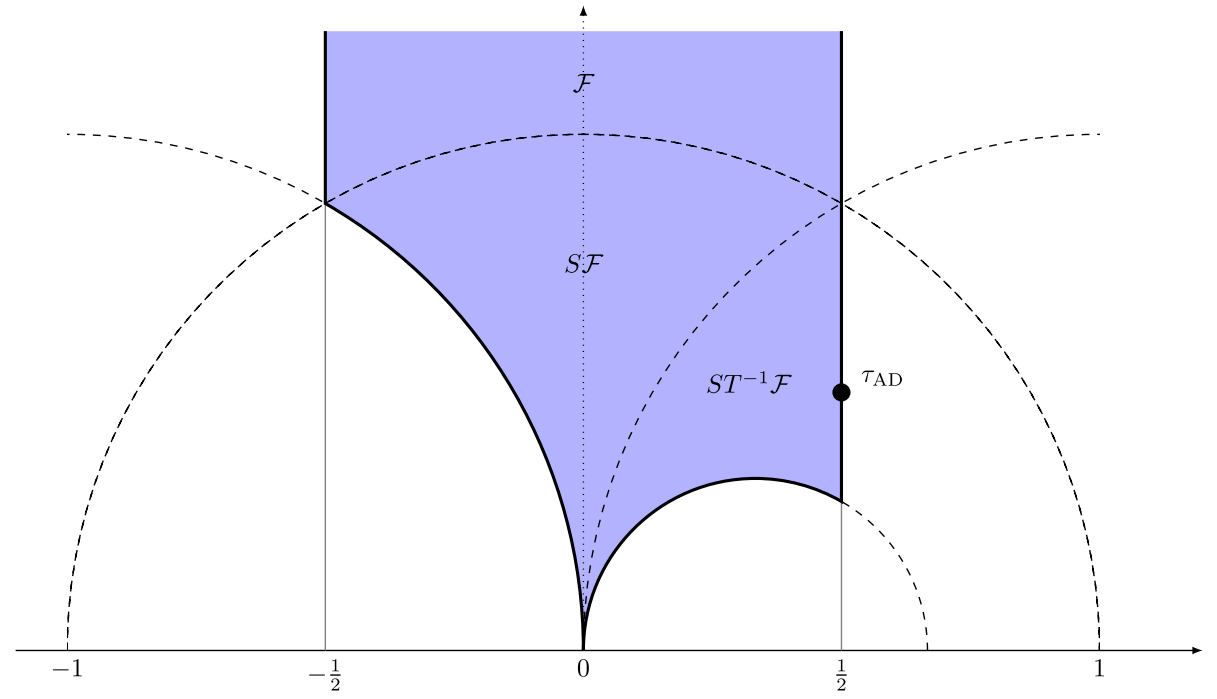

FIG. 21. Fundamental domain of $\Gamma_{0}(2)$. This is the duality group of $N_{f}=3$ with mass $\boldsymbol{m}=\frac{1}{16}\left(\Lambda_{3}, 0,0\right)$. The AD point is the elliptic fixed point of the domain, located at $\tau_{\mathrm{AD}}=\frac{1}{2}+\frac{i}{2}$.

\section{F. Type II AD mass}

On the equal mass $\boldsymbol{m}=(m, m, m)$ curve we can also tune the mass to $m=m_{\mathrm{AD}}=-\frac{1}{64} \Lambda_{3}$ to find a type II AD theory at $u_{\mathrm{AD}}=\frac{5}{2^{10}} \Lambda_{3}^{2}$. By fixing the mass to this value we find

$$
\frac{u(\tau)}{\Lambda_{3}^{2}}=-\frac{f_{3 \mathrm{~B}}(3 \tau)+7}{2^{12}}
$$

with $f_{3 \mathrm{~B}}$ given in Eq. (4.10). At $u_{\mathrm{AD}}$ two mutually nonlocal singularities collide, while the other three reside at $u_{0}=-\frac{7}{2^{12}} \Lambda_{3}^{2}$. The physical discriminant is therefore
$\Delta=\left(u-u_{\mathrm{AD}}\right)^{2}\left(u-u_{0}\right)^{3}$. We know from Sec. IV B that $\tau \mapsto f_{3 \mathrm{~B}}(3 \tau)$ is a Hauptmodul for $\Gamma_{0}(3)$, and in fact the fundamental domain is just given by the one for $\Gamma^{0}(3)$ as in Fig. 6, with every point divided by 3 . It also decomposes into $\operatorname{SL}(2, \mathbb{Z})$ images of $\mathcal{F}$, see Fig. 22.

The AD point $u\left(\tau_{\mathrm{AD}}\right)=u_{\mathrm{AD}}$ translates to $f_{3 \mathrm{~B}}\left(3 \tau_{\mathrm{AD}}\right)=$ -27 which has $\tau_{\mathrm{AD}}=\frac{1}{\sqrt{3}} \omega_{12}$ as a solution (where $\left.\omega_{j}=e^{2 \pi i / j}\right)$. The other singularity satisfies $f_{3 \mathrm{~B}}(3 \tau)=0$ and therefore $\tau=0$. In terms of the Hauptmodul of $\Gamma_{0}(3)$ the $j$ invariant of the curve with above given mass $\boldsymbol{m}_{\mathrm{AD}}$ reads $j=\left(f_{3 \mathrm{~B}}+27\right)\left(f_{3 \mathrm{~B}}+243\right)^{3} / f_{3 \mathrm{~B}}^{3}$. This proves that the $\mathrm{AD}$ singularity is Kodaira type II and therefore indeed

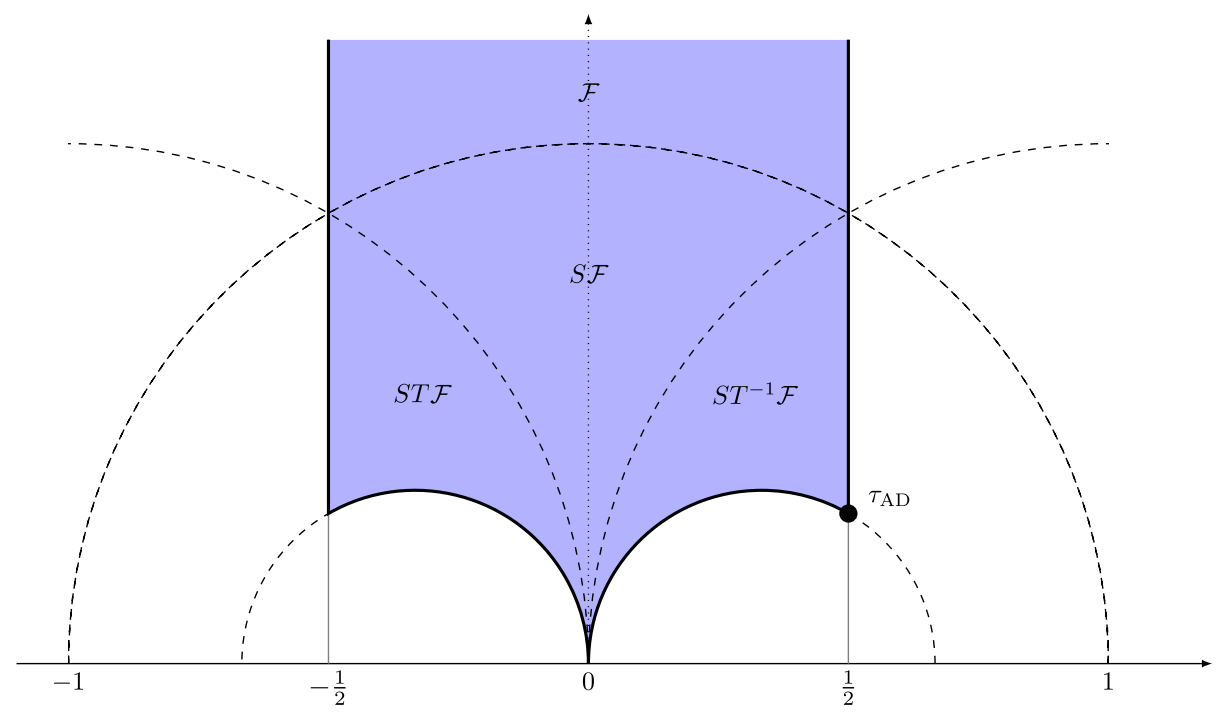

FIG. 22. Fundamental domain of $\Gamma_{0}(3)$, the duality group of $N_{f}=3$ with mass $\boldsymbol{m}=-\frac{1}{64}\left(\Lambda_{3}, \Lambda_{3}, \Lambda_{3}\right)$. It has index 4 in PSL(2, $\left.\mathbb{Z}\right)$ and a type II AD point located at the elliptic fixed point $\tau_{\mathrm{AD}}=\frac{\omega_{12}}{\sqrt{3}}=\frac{1}{2}+\frac{1}{2 \sqrt{3}} i$. The width of the cusp $\tau=0$ is 3 . 
equivalent to the II theory in $N_{f}=1$, see Sec. IV B. It is interesting that both curves are parametrized by the same Hauptmodul, as the number of singularities on the curves are different.

The monodromies are given by

$$
\begin{aligned}
M_{0} & =S T^{3} S^{-1}=\left(\begin{array}{cc}
1 & 0 \\
-3 & 1
\end{array}\right), \\
M_{\mathrm{AD}} & =(T S)^{-1}(S T)^{-1}(T S)=\left(\begin{array}{ll}
-1 & 1 \\
-3 & 2
\end{array}\right),
\end{aligned}
$$

which are just (4.17) conjugated by $\operatorname{diag}(3,1)$. They furthermore satisfy $M_{0} M_{\mathrm{AD}}=M_{\infty}$ with $M_{\infty}=P T^{-1}$. Since $M_{\mathrm{AD}}^{6}=\mathbb{1}$ in $\operatorname{PSL}(2, \mathbb{Z})$, the $\mathrm{AD}$ point $\tau_{\mathrm{AD}}$ is an elliptic fixed point in $\Gamma_{0}(3)$. Its stabilizer $M_{\mathrm{AD}}$ decomposes into the monodromy $(S T)^{-1}$ around $\tau=\omega_{3}$, and the path $(T S)^{-1}: \omega_{3} \mapsto \tau_{\mathrm{AD}}$.

\section{DISCUSSION}

We have studied the Coulomb branches $\mathcal{B}_{N_{f}}$ of $S U(2)$ $\mathcal{N}=2$ Yang-Mills theories with $N_{f} \leq 3$ massive hypermultiplets in the fundamental representation. In particular, we have considered the order parameter $u$ as function of the effective coupling, and derive domains $\mathcal{F}_{N_{f}}$ such that $u: \mathcal{F}_{N_{f}} \rightarrow \mathcal{B}_{N_{f}}$ is one to one. We find that generically the function $u$ has square roots appearing in the expressions for $u$, such that $\mathcal{F}_{N_{f}}$ is not isomorphic to a domain $\Gamma \backslash \mathbb{W}$ for a congruence subgroup of $\mathrm{SL}(2, \mathbb{Z})$. Nevertheless, exact expressions can be determined, such as for $N_{f}=2$ with two equal masses, and $N_{f}=3$ with one nonvanishing mass. For other special values, branch points and cuts can be absent and the fundamental domain is that of a modular curve for a congruence subgroups of $\operatorname{SL}(2, \mathbb{Z})$, as also encountered in cases in the literature [3,28-30].

We described how the order parameters are naturally expressed as roots of certain degree six polynomials with modular functions as coefficients. Many interesting aspects of the theories can be read off from these polynomials:

(i) The degree of the polynomial tells us that the fundamental domains of the order parameters can be described as six copies of the ordinary $\operatorname{SL}(2, \mathbb{Z})$ domain.

(ii) For the modular theories this further implies that the duality group needs to be at most index 6 in $\operatorname{SL}(2, \mathbb{Z})$.

(iii) The discriminant of the sextic polynomials includes the branch points as well as the superconformal AD fixed points of the theories.

(iv) We further discussed how one can explicitly construct fundamental regions of order parameters as images of fundamental domains in $\mathcal{F}_{N_{f}}$. The partitioning of the fundamental regions of the order parameters seem to generalize aspects of the dessins d'enfants $[49,59-62,89,94,95]$ to the case of nonmodular elliptic surfaces.

Physically, the branch points and cuts provide a mechanism for $\mathcal{F}_{N_{f}}$ to evolve as function of the mass. This is most apparent in the limits where a hypermultiplet decouples or multiple singularities coincide, where branch cuts appear to "cut" and "glue" regions of $\mathcal{F}_{N_{f}}$. In particular near an $\mathrm{AD}$ point, regions with nonlocal cusps are disconnected from $\mathcal{F}_{N_{f}}$. This makes it manifest that, on the $u$ plane, not only nonlocal singularities become coincident but also branch points, which ceases to be branch points in the limit because also the preimages in $\mathcal{F}_{N_{f}}$ have collided.

We believe that our methods can be adapted to many other rank one theories, such as those of class $\mathcal{S}[46,96]$. The present analyses could perhaps also be used to draw lessons about moduli spaces of other theories, such as $\mathcal{N}=2$ SYM with gauge group $S U(N)$ for $N>2$ or Calabi-Yau compactifications in string theory, where in many cases similar structures should arise. Remnants of which could perhaps be seen in $[78,79]$. Lastly, we hope our methods find applications in similar geometries such as F theory [97] and 5D supersymmetric conformal field theories [98]. Moreover, our findings may benefit the evaluation of the $u$-plane integral $[11,17,18,21,99]$. We aim to address this integral for massive $\mathcal{N}=2 \mathrm{QCD}$ in future work [70].

We would further like to mention to explore potential physical consequences of the branch points. It is known that the $\mathrm{AD}$ points correspond to critical points of a second order phase transition [84,100,101]. It might then be natural to think of the branch cuts in $\mathcal{F}_{N_{f}}$, as in for example Fig. 7, as boundaries over which a first order phase transition takes place. Since branch points and cuts seem to be a generic feature, it would suggest that similar points appear in all theories with these kinds of superconformal fixed points. It would of course be very interesting to study this further and we leave that for future work.

Another potential application is the $\mathcal{N}=2$ QCD beta function. In Refs. [42,43], Dolan gave a proposal for the beta function of the massless $N_{f}=0,1,2,3$ theories, expanding on the work of [31,102-105]. For the cases $N_{f}=0,2,3$ we can use the notation of the present paper to collect these conjectured expressions as ${ }^{15}$

$$
\beta_{N_{f}}=-\hat{\Delta} \frac{1}{u} \frac{d \tau}{d u}
$$

where $\hat{\Delta}$ is the reduced discriminant of (3.19). In Ref. [43] a shift is also made for $u$ in $N_{f}=3$. For $N_{f}=1$ the argument is revised, basically due to the square roots appearing in $u$ and the spoilage of modularity. We will

\footnotetext{
${ }^{15}$ Compare with Eqs. (2.15), (3.11) and (4.21) of Ref. [43].
} 
disregard these details in the following discussion. Using our knowledge from the present paper it is now natural to conjecture that the beta functions of the general massive theories, at least for $N_{f}=2$ and 3 , is given by

$$
\beta_{N_{f}}=-\frac{\Delta}{P_{N_{f}}^{\mathrm{M}}} \frac{1}{u} \frac{d \tau}{d u}=\frac{4-N_{f}}{16 \pi i} \frac{1}{u}\left(\frac{d u}{d a}\right)^{2},
$$

where the Matone relation was used in the second step. This obviously gives back the expression (7.1) for the massless $N_{f}=0,2,3$. It furthermore satisfies many good qualities, such as being a weight -2 modular form in the cases where the theories are modular. For the theories where we have explicit expressions for the relevant quantities we can explicitly check that it has the correct behavior near the singular points. This is done in Appendix C. This proposal is speculative but would serve as an interesting topic for further investigations.

\section{ACKNOWLEDGMENTS}

We are happy to thank Ling Long, Gregory Moore, Ken Ono and Edward Witten for correspondence and discussions. J. A. is supported by the Government of Ireland Postgraduate Scholarship Programme Grant No. GOIPG/ 2020/910 of the Irish Research Council. E. F. is supported by the TCD Provost's PhD Project Award. J. M. is supported by the Laureate Award 15175 "Modularity in Quantum Field Theory and Gravity" of the Irish Research Council. This research was supported in part by the National Science Foundation under Grant No. NSF PHY-1748958 through the KITP program "Modularity in Quantum Systems.”
Note added.-While completing this paper, the work [106] by Closset and Magureanu appeared on the arXiv with partial overlapping results on modular fundamental domains within $\mathcal{N}=2$ QCD.

\section{APPENDIX A: ELLIPTIC CURVES AND AUTOMORPHIC FORMS}

We collect some properties of modular forms for subgroups of $S L(2, \mathbb{Z})$ here. For further reading see for example $[68,81,107-110]$.

\section{Modular forms}

We make use of modular forms for the congruence subgroups $\Gamma_{0}(n)$ and $\Gamma^{0}(n)$ of $S L(2, \mathbb{Z})$. They are defined as

$$
\begin{aligned}
& \Gamma_{0}(n)=\left\{\left(\begin{array}{ll}
a & b \\
c & d
\end{array}\right) \in S L(2, \mathbb{Z}) \mid c \equiv 0 \quad \bmod n\right\}, \\
& \Gamma^{0}(n)=\left\{\left(\begin{array}{ll}
a & b \\
c & d
\end{array}\right) \in S L(2, \mathbb{Z}) \mid b \equiv 0 \quad \bmod n\right\},
\end{aligned}
$$

and are related by conjugation with the matrix $\operatorname{diag}(n, 1)$. We furthermore define the principal congruence subgroup $\Gamma(n)$ as the subgroup of $S L(2, \mathbb{Z}) \ni A$ with $A \equiv \mathbb{1} \bmod n$. A subgroup $\Gamma$ of $\operatorname{SL}(2, \mathbb{Z})$ is called a congruence subgroup if there exists an integer $n \in \mathbb{N}$ such that it contains $\Gamma(n)$. The smallest such $n$ is then called the level of $\Gamma$.

The Jacobi theta functions $\vartheta_{j}: \mathbb{\sharp} \rightarrow \mathbb{C}, j=2,3,4$, are defined as

$$
\vartheta_{2}(\tau)=\sum_{r \in \mathbb{Z}+\frac{1}{2}} q^{r^{2} / 2}, \quad \vartheta_{3}(\tau)=\sum_{n \in \mathbb{Z}} q^{n^{2} / 2}, \quad \vartheta_{4}(\tau)=\sum_{n \in \mathbb{Z}}(-1)^{n} q^{n^{2} / 2},
$$

with $q=e^{2 \pi i \tau}$. These functions transform under $T, S \in S L(2, \mathbb{Z})$ as

$$
\begin{aligned}
& S: \vartheta_{2}(-1 / \tau)=\sqrt{-i \tau} \vartheta_{4}(\tau), \quad \vartheta_{3}(-1 / \tau)=\sqrt{-i \tau} \vartheta_{3}(\tau), \quad \vartheta_{4}(-1 / \tau)=\sqrt{-i \tau} \vartheta_{2}(\tau) \\
& T: \vartheta_{2}(\tau+1)=e^{\frac{\pi i}{4}} \vartheta_{2}(\tau), \quad \vartheta_{3}(\tau+1)=\vartheta_{4}(\tau), \quad \vartheta_{4}(\tau+1)=\vartheta_{3}(\tau) .
\end{aligned}
$$

They furthermore satisfy the Jacobi abstruse identity

$$
\vartheta_{2}^{4}+\vartheta_{4}^{4}=\vartheta_{3}^{4}
$$

Derivatives of modular functions are described by Ramanujan's differential operator. It increases the holomorphic weight by 2 and it can be explicitly constructed using the theory of Hecke operators [108]. For the derivatives of the Jacobi theta functions, one finds

$$
\begin{aligned}
& D \vartheta_{2}^{4}=\frac{1}{6} \vartheta_{2}^{4}\left(E_{2}+\vartheta_{3}^{4}+\vartheta_{4}^{4}\right), \\
& D \vartheta_{3}^{4}=\frac{1}{6} \vartheta_{3}^{4}\left(E_{2}+\vartheta_{2}^{4}-\vartheta_{4}^{4}\right), \\
& D \vartheta_{4}^{4}=\frac{1}{6} \vartheta_{4}^{4}\left(E_{2}-\vartheta_{2}^{4}-\vartheta_{3}^{4}\right),
\end{aligned}
$$

where $D:=\frac{1}{2 \pi i} \frac{d}{d \tau}=q \frac{d}{d q}$ and $E_{2}$ is the quasimodular Eisenstein series (A13) of weight 2, transforming as (A14). 
The modular lambda function $\lambda=\frac{\vartheta_{2}^{4}}{\vartheta_{3}^{4}}$ is a Hauptmodul for $\Gamma(2)$. The Dedekind eta function $\eta: \mathbb{H} \rightarrow \mathbb{C}$ is defined as the infinite product

$$
\eta(\tau)=q^{\frac{1}{24}} \prod_{n=1}^{\infty}\left(1-q^{n}\right), \quad q=e^{2 \pi i \tau} .
$$

It transforms under the generators of $S L(2, \mathbb{Z})$ as

$$
\begin{aligned}
& S: \eta(-1 / \tau)=\sqrt{-i \tau} \eta(\tau), \\
& T: \eta(\tau+1)=e^{\frac{\pi i}{2}} \eta(\tau),
\end{aligned}
$$

and relates to the Jacobi theta series as $\eta^{3}=\frac{1}{2} \vartheta_{2} \vartheta_{3} \vartheta_{4}$. The derivative of $\eta$ is given by $\eta^{\prime}=\frac{\pi i}{12} \eta E_{2}$.

Another class of theta series is provided by the one of the $A_{2}$ root lattice, $b_{3, j}: \mathbb{W} \rightarrow \mathbb{C}$,

$$
b_{3, j}(\tau)=\sum_{k_{1}, k_{2} \in \mathbb{Z}+\frac{j}{3}} q^{k_{1}^{2}+k_{2}^{2}+k_{1} k_{2}}, \quad j \in\{-1,0,1\} .
$$

It is clear that $b_{3,-1}=b_{3,1}$. The transformation properties under $\operatorname{SL}(2, \mathbb{Z})$ are

$$
\begin{aligned}
S: b_{3, j}\left(-\frac{1}{\tau}\right) & =-\frac{i \tau}{\sqrt{3}}, \sum_{\bmod 3} \omega_{3}^{2 j l} b_{3, l}(\tau), \\
T: b_{3, j}(\tau+1) & =\omega_{3}^{j^{2}} b_{3, j}(\tau) .
\end{aligned}
$$

The $b_{3, j}$ series can be expressed through the Dedekind eta function (A7) as

$b_{3,0}(\tau)=\frac{\eta\left(\frac{\tau}{3}\right)^{3}+3 \eta(3 \tau)^{3}}{\eta(\tau)}, \quad b_{3,1}(\tau)=3 \frac{\eta(3 \tau)^{3}}{\eta(\tau)}$.

It furthermore relates to the quasimodular Eisenstein series $E_{2}$ by

$$
E_{2}\left(\frac{\tau}{3}\right)-3 E_{2}(\tau)=-2 b_{3,0}\left(\frac{\tau}{3}\right)^{2}
$$

A relation to the Jacobi theta functions is given by

$$
b_{3,0}(\tau)=\vartheta_{3}(2 \tau) \vartheta_{3}(6 \tau)+\vartheta_{2}(2 \tau) \vartheta_{2}(6 \tau) .
$$

Quotients of $\eta$ functions are frequently used to generate bases for the spaces of modular functions for congruence subgroups of $S L(2, \mathbb{Z})$. We use the following

Theorem 1. ([108,111]) Let $f(\tau)=\prod_{\delta \mid N} \eta(\delta \tau)^{r_{\delta}}$ be an $\eta$ quotient with $k=\frac{1}{2} \sum_{\delta \mid N} r_{\delta} \in \mathbb{Z}$ and $\sum_{\delta \mid N} \delta r_{\delta} \equiv$ $\sum_{\delta \mid N} \frac{N}{\delta} r_{\delta} \equiv 0 \bmod 24$. Then, $f$ is a weakly holomorphic modular form for $\Gamma_{0}(N)$ with weight $k$.

\section{a. Eisenstein series}

We let $\tau \in \mathbb{U}$ and define $q=e^{2 \pi i \tau}$. Then the Eisenstein series $E_{k}: \mathbb{H} \rightarrow \mathbb{C}$ for even $k \geq 2$ are defined as the $q$ series

$$
E_{k}(\tau)=1-\frac{2 k}{B_{k}} \sum_{n=1}^{\infty} \sigma_{k-1}(n) q^{n}
$$

with $\sigma_{k}(n)=\sum_{d \mid n} d^{k}$ the divisor sum. For $k \geq 4$ even, $E_{k}$ is a modular form of weight $k$ for $S L(2, \mathbb{Z})$. On the other hand $E_{2}$ is a quasimodular form, which means that the $S L(2, \mathbb{Z})$ transformation of $E_{2}$ includes a shift in addition to the weight,

$E_{2}\left(\frac{a \tau+b}{c \tau+d}\right)=(c \tau+d)^{2} E_{2}(\tau)-\frac{6 i}{\pi} c(c \tau+d)$.

From the $S$ transformation, we find that

$$
E_{4}\left(e^{\pi i / 3}\right)=0, \quad E_{6}(i)=0,
$$

and the zeros are unique in $S L(2, \mathbb{Z}) \backslash \mathbb{W}$ according to the valence formula for modular forms on $S L(2, \mathbb{Z})$. Any modular form for $S L(2, \mathbb{Z})$ can be related to the Jacobi theta functions (A2) by

$$
\begin{aligned}
& E_{4}=\frac{1}{2}\left(\vartheta_{2}^{8}+\vartheta_{3}^{8}+\vartheta_{4}^{8}\right), \\
& E_{6}=\frac{1}{2}\left(\vartheta_{2}^{4}+\vartheta_{3}^{4}\right)\left(\vartheta_{3}^{4}+\vartheta_{4}^{4}\right)\left(\vartheta_{4}^{4}-\vartheta_{2}^{4}\right) .
\end{aligned}
$$

All quasimodular forms for $S L(2, \mathbb{Z})$ can be expressed as polynomials in $E_{2}, E_{4}$ and $E_{6}$. The derivatives of the Eisenstein series are quasimodular,

$E_{2}^{\prime}=\frac{2 \pi i}{12}\left(E_{2}^{2}-E_{4}\right), \quad E_{4}^{\prime}=\frac{2 \pi i}{3}\left(E_{2} E_{4}-E_{6}\right)$,

$E_{6}^{\prime}=\frac{2 \pi i}{2}\left(E_{2} E_{6}-E_{4}^{2}\right)$.

These equations give the differential ring structure of quasimodular forms on $\operatorname{PSL}(2, \mathbb{Z})$. With our normalization (A13) the $j$ invariant can be written as

$$
j=1728 \frac{E_{4}^{3}}{E_{4}^{3}-E_{6}^{2}}=256 \frac{\left(\vartheta_{3}^{8}-\vartheta_{3}^{4} \vartheta_{4}^{4}+\vartheta_{4}^{8}\right)^{3}}{\vartheta_{2}^{8} \vartheta_{3}^{8} \vartheta_{4}^{8}} .
$$

\section{Modular curves}

A subgroup $\Gamma$ of $\operatorname{SL}(2, \mathbb{Z})$ is a congruence subgroup if $\Gamma \supset \Gamma(N)$ for some $N \in \mathbb{N}$, which is called the level of $\Gamma$. The (projective) index of a congruence subgroup $\Gamma$ is defined as

$$
\operatorname{ind} \Gamma=[\operatorname{PSL}(2, \mathbb{Z}): \Gamma] \text {, }
$$


and it is finite for all $N$. By $\operatorname{SL}(2, \mathbb{Z})$ we strictly mean $\operatorname{PSL}(2, \mathbb{Z})$ in the following. In fact, one can prove [68]

$$
\operatorname{ind} \Gamma(N)=N^{3} \prod_{p \mid N}\left(1-\frac{1}{p^{2}}\right), \quad \operatorname{ind} \Gamma^{0}(N)=N \prod_{p \mid N}\left(1+\frac{1}{p}\right),
$$

where the sum is over all prime divisors of $N$. It can also be computed in the following way. The volume of the curve $\Gamma \backslash \mathbb{M}$ is defined as

$$
\operatorname{vol}(\Gamma \backslash \mathbb{M})=\int_{\Gamma \backslash \boxplus} \mathrm{d} \mu,
$$

where $\mathrm{d} \mu=y^{-2} \mathrm{~d} x \mathrm{~d} y$ is the hyperbolic metric on $\mathbb{U}$, with $\tau=x+i y$. Since $\operatorname{vol}(\operatorname{SL}(2, \mathbb{Z}) \backslash \mathbb{U})=\frac{\pi}{3}$ can easily be computed, the index of any $\Gamma \subseteq \operatorname{SL}(2, \mathbb{Z})$ is then given by

$$
\operatorname{ind} \Gamma=\frac{3}{\pi} \operatorname{vol}(\Gamma \backslash \mathbb{M}) \text {. }
$$

Let $\Gamma$ be a congruence subgroup of $\operatorname{SL}(2, \mathbb{Z})$. Cusps of $\Gamma$ are $\Gamma$-equivalence classes of $\mathbb{Q} \cup\{\infty\}$. Adjoining coordinate charts to the cusps and compactifying gives the modular curve $X(\Gamma):=\Gamma \backslash(\mathbb{Q} \cup \mathbb{Q} \cup\{i \infty\})$. The isotropy (stabilizer) group of $\infty$ in $\operatorname{SL}(2, \mathbb{Z})$ is the Abelian group of translations,

$$
\operatorname{SL}(2, \mathbb{Z})_{\infty}=\left\{\left(\begin{array}{ll}
1 & m \\
0 & 1
\end{array}\right): m \in \mathbb{Z}\right\} .
$$

For each cusp $s \in \mathbb{Q} \cup\{i \infty\}$ some $\delta_{s} \in \operatorname{SL}(2, \mathbb{Z})$ maps $s \mapsto \infty$. The width of $s$ is defined as

$$
h_{\Gamma}(s)=\left|\mathrm{SL}(2, \mathbb{Z})_{\infty} /\left(\delta_{s} \Gamma \delta_{s}^{-1}\right)_{\infty}\right| .
$$

It can be proven that this definition is independent of $\delta_{s}$. For a fixed group $\Gamma$ it can be viewed as a well-defined function $\mathbb{Q} \cup\{i \infty\} \rightarrow \mathbb{N}_{0}$. It is straightforward to show that the sum over the widths of all inequivalent cusps $\mathcal{C}$ is equal to the index [112]

$$
\sum_{s \in \mathcal{C}_{\Gamma}} h_{\Gamma}(s)=\operatorname{ind} \Gamma
$$

The width of 0 (which is the level) is the least common divisor of all widths, and the width of $\infty$ is the gcd of all widths.

Other invariants of modular curves are the elliptic fixed points. A point $\tau \in \mathbb{U}$ is an elliptic point for $\Gamma$ if its isotropy (stabilizer) group is nontrivial. The period of $\tau$ is defined as the order of the isotropy group. It can be shown that any congruence subgroup of $\operatorname{SL}(2, \mathbb{Z})$ has only finitely many elliptic points, and the period for any point $\tau \in \mathbb{U}$ is 1,2 or 3 .

\section{a. Riemann-Hurwitz formula}

Let $f: X \rightarrow Y$ be a nonconstant holomorphic map between compact Riemann surfaces $X, Y$. It has a degree $n \in \mathbb{N}$, such that $\left|f^{-1}(y)\right|=n$ for all but finitely many $y \in Y$. More precisely, for each point $x \in X$ let $e_{x} \in \mathbb{N}$ be the ramification degree of $f$ at $x$, i.e. the multiplicity with which $f$ takes 0 to 0 as a map in local coordinates, making $f$ an $e_{x}$-to-1 map around $x$. Then there exists a positive integer $n$ such that

$$
\sum_{x \in f^{-1}(y)} e_{x}=n
$$

for all $y \in Y$. If $g_{X}$ and $g_{Y}$ are the genera of $X$ and $Y$, then the Riemann-Hurwitz formula

$$
2 g_{X}-2=n\left(2 g_{Y}-2\right)+\sum_{x \in X}\left(e_{x}-1\right)
$$

states that the Euler characteristic of $X$ is that of $Y$ multiplied by the degree $n$ of the cover, corrected by contributions from the ramification points. It is obvious that $g_{X} \geq g_{Y}$, otherwise $f$ is not holomorphic.

This allows to compute the genus of a modular curve $X(\Gamma)$ for any congruence subgroup $\Gamma \subseteq \operatorname{SL}(2, \mathbb{Z})$. For this, let $X=X(\Gamma)$ and $Y=X(1)$. Let $y_{2}=\operatorname{SL}(2, \mathbb{Z}) \cdot i, y_{3}=$ $\operatorname{SL}(2, \mathbb{Z}) \cdot e^{\frac{\pi i}{3}}$ and $\varepsilon_{2}$ and $\varepsilon_{3}$ be the number of elliptic fixed points of period 2 and 3 for $X(\Gamma)$, and finally $y_{\infty}=$ $\operatorname{SL}(2, \mathbb{Z}) \cdot \infty$ and $\varepsilon_{\infty}$ be the number of cusps $X(\Gamma)$. Then $e_{x}-1$ is only nonzero when $x \in f^{-1}\left(y_{h}\right)$ for $h=2,3, \infty$. Since $g_{X(1)}=0$, it follows from (A27) that

$$
g=1+\frac{n}{12}-\frac{\varepsilon_{2}}{4}-\frac{\varepsilon_{3}}{3}-\frac{\varepsilon_{\infty}}{2},
$$

where $g=g_{X(\Gamma)}$ and $n=$ ind $\Gamma$. See, for example, Ref. [68] for a more detailed derivation of this formula.

Conjugacy classes of subgroups of $\operatorname{SL}(2, \mathbb{Z})$ can be classified by the data $\left(n, \varepsilon_{\infty}, \varepsilon_{2}, \varepsilon_{3}\right)$ together with the set of widths of the $\varepsilon_{\infty}$ cusps. For instance, the subgroups of $\operatorname{SL}(2, \mathbb{Z})$ of index $n=6$ have been completely classified $[81,113]$. There are precisely 22 subgroups that fall into 8 conjugacy classes, and they are listed in Table II. It has been shown in Ref. [114] that every subgroup of $\operatorname{SL}(2, \mathbb{Z})$ with index $n \leq 6$ is a congruence subgroup. Examples of noncongruence subgroups of $\operatorname{SL}(2, \mathbb{Z})$ of index 7 have been constructed already in the 19th century by Fricke. Hauptmoduln for low index subgroups of $\operatorname{SL}(2, \mathbb{Z})$ are studied in more detail in Refs. [50,115].

\section{Kodaira classification}

Let us consider an elliptic curve in Weierstraß form,

$$
y^{2}=4 x^{3}-g_{2} x-g_{3},
$$


TABLE II. Classification of conjugacy classes of index 6 subgroups of $\operatorname{SL}(2, \mathbb{Z})$ [81]. The first column gives the number of cusps and elliptic fixed points of order 2 and 3 . According to the Riemann-Hurwitz formula (A28) all but the $(1,0,0)$ one induce genus 0 modular curves, whose Hauptmodul $x$ is expressed in terms of the $j$ invariant. In fact the group with signature $(1,0,0)$ is the only subgroup of $\operatorname{SL}(2, \mathbb{Z})$ of nonzero genus and index $\leq 7$. The second column gives the indices of the $\varepsilon_{\infty}$ number of cusps, and the last column counts the number of conjugate groups with the same data. From the expression of $j=\frac{p(x)}{q(x)}$ as a rational function in $x$ we learn that $\operatorname{deg} p=n$ gives the index and the difference $\operatorname{deg} p-\operatorname{deg} q=h(\infty)$ gives the width of $\infty$. The roots of $q(x)=0$ then give the cusps in $\mathbb{Q}$ with widths provided by their corresponding multiplicity.

\begin{tabular}{lccc}
\hline \hline$\left(\varepsilon_{\infty}, \varepsilon_{2}, \varepsilon_{3}\right)$ & Cusps & Hauptmodul & |conj \\
\hline$(1,0,0)$ & 6 & $g=1$ & 1 \\
$(1,0,3)$ & 6 & $j=-27 x^{3}\left(x^{3}+16\right)$ & 2 \\
$(1,4,0)$ & 6 & $j=27\left(x^{2}+4\right)^{3}$ & 3 \\
$(2,2,0)$ & $3+3$ & $j=\frac{x^{3}(x+12)^{3}}{(x+9)^{3}}$ & 3 \\
$(2,2,0)$ & $4+2$ & $j=\frac{x^{3}(x+8)^{3}}{(x+4)^{2}}$ & 3 \\
$(2,2,0)$ & $5+1$ & $j=\frac{\left(x^{2}+10 x+5\right)^{3}}{x}$ & 6 \\
$(3,0,0)$ & $2+2+2$ & $j=\frac{\left(x^{2}+192\right)^{3}}{\left(x^{2}-64\right)^{2}}$ & 1 \\
$(3,0,0)$ & $4+1+1$ & $j=\frac{\left(x^{2}+48\right)^{3}}{x^{2}+64}$ & 3 \\
\hline \hline
\end{tabular}

where $g_{2}$ and $g_{3}$ are functions of some parameter $u$. This is the case for all SW curves (2.3), where $g_{2}$ and $g_{3}$ are polynomials of degree 2 and 3 in $u$. The discriminant of the Weierstra $\beta$ curve is $\Delta=g_{2}^{3}-27 g_{3}^{2}$. The study of the elliptic curves on the discriminant divisor $\Sigma=\{u \mid \Delta(u)=$ $0\}$ is the famous Kodaira classification. At a generic point in $\Sigma, g_{2}$ and $g_{3}$ do not vanish simultaneously. If we denote by ord $f$ the order of vanishing (order of zero) of a polynomial or power series $f$ at a given point, then on a generic point in $\Sigma$ we have ord $\left(g_{2}, g_{3}, \Delta\right)=(0,0,1)$. The various combinations of orders of vanishing of the invariants $g_{2}$ and $g_{3}$ are classified $[63,64]$, see for a review [116]. At special points in $\Sigma$ both $g_{2}=g_{3}=0$. From (A29) we see that the curve becomes cuspidal, $y^{2}=x^{3}$. However the shape of the curve depends on the orders of vanishing of the respective quantities. Part of

TABLE III. Part of the Kodaira-Tate table for singular fibers of the Weierstraß model.

\begin{tabular}{lccc}
\hline \hline Type & ord $g_{2}$ & ord $g_{3}$ & ord $\Delta$ \\
\hline$I_{0}$ & $\geq 0$ & $\geq 0$ & 0 \\
$I_{1}$ & 0 & 0 & 1 \\
$I I$ & $\geq 1$ & 1 & 2 \\
$I I I$ & 1 & $\geq 2$ & 3 \\
$I V$ & $\geq 2$ & 2 & 4 \\
$I_{m}$ & 0 & 0 & $m$ \\
\hline \hline
\end{tabular}

the classification is given in Table III. The singular fibers of the Kodaira classification are all realized as curves of physical theories $[117,118]$.

\section{APPENDIX B: ADDITIONAL PROOF OF MATONE'S RELATION}

We can give a second proof of the Matone's relation (3.13). As the behavior of the polynomials (3.15) is highly constrained, they allow us to give another proof of the $N_{f} \leq 3$ Matone relation (3.13). For this, first note that the rhs $X:=\left(\frac{d u}{d a}\right)^{2} \frac{d u}{d \tau}$ has mass dimension $\Lambda_{N_{f}}^{4}$. In the massless $0 \leq N_{f} \leq 3$ theories, $X$ can be written as $-\frac{16 \pi i}{4-N_{f}} \frac{\Delta_{N_{f}}}{Q_{N_{f}}}$ where $\Delta_{N_{f}}$ is the physical discriminant and $Q_{N_{f}}$ a polynomial, both monic in $u$. This fixes the overall normalization. Since $\Delta_{N_{f}}$ has degree $N_{f}+2$ for any mass, dimensional analysis fixes $\operatorname{deg} Q_{N_{f}}=N_{f}$. We are left with determining $Q_{N_{f}}$ and proving that indeed $Q_{N_{f}}=P_{N_{f}}^{\mathrm{M}}$.

The relation

$$
\frac{d u}{d \tau}=-\frac{16 \pi i}{4-N_{f}} \frac{\Delta_{N_{f}}}{Q_{N_{f}}}\left(\frac{d a}{d u}\right)^{2}
$$

is renormalization group ( $R G)$ invariant since its ingredients are. For instance, both $\frac{d u}{d \tau}$ and $\frac{d a}{d u}$ flow from $N_{f}$ to $N_{f}-1$ by decoupling a hypermultiplet of mass $m_{i}$. This is not true for both $\Delta_{N_{f}}$ or $Q_{N_{f}}$, since their degrees as polynomials in $u$ are reduced by 1 by decoupling a multiplet. However, it is easy to check that

$$
\Delta_{N_{f}}=u^{N_{f}+2}-u^{N_{f}+1} \sum_{i=1}^{N_{f}} m_{i}^{2}+\mathcal{O}\left(u^{N_{f}}\right)
$$

as $u \rightarrow \infty$. This implies that $\operatorname{deg} \lim _{m_{i} \rightarrow \infty} \frac{\Delta_{N_{f}}}{m_{i}^{2}}=\operatorname{deg} \Delta_{N_{f}}-$ $1=\operatorname{deg} \Delta_{N_{f}-1}$. In fact, one can check explicitly that

$$
\lim _{m_{i} \rightarrow \infty} \frac{\Delta_{N_{f}}}{m_{i}^{2}}=-\Delta_{N_{f}-1} .
$$

The reason for this is that on the singular locus $\Delta_{N_{f}}=0$ there is always a singularity $u_{*}$ that behaves as $u_{*} \sim m_{i}^{2}$ for $m_{i} \rightarrow \infty$, it is the singularity that decouples (see also Ref. [119] for a pictorial description). The relation (B1) is then RG invariant if and only if

$$
\lim _{m_{i} \rightarrow \infty} \frac{Q_{N_{f}}}{m_{i}^{2}}=-\frac{4-\left(N_{f}-1\right)}{4-N_{f}} Q_{N_{f-1}},
$$

for $N_{f}>0$. This condition does not give enough constraints on $Q_{N_{f}}$ in general to fully determine it. However, (B4) together with $\operatorname{deg} Q_{N_{f}}=N_{f}$, $\left[Q_{N_{f}}\right]=\Lambda_{N_{f}}^{2 N_{f}}$ and the 
symmetry in the masses (or equivalently, the freedom of choice of $m_{i}$ ) restricts $Q_{N_{f}}$ to be a polynomial where each coefficient has finitely many terms. The decoupling (B4) as well as limits to known examples fix all of these coefficients. For instance, for $N_{f}=0$ it is known that $Q_{1}=1$. For $N_{f}=1$, we can make a general ansatz

$$
Q_{1}=u+c_{1} m_{1}^{2}+c_{2} m_{1} \Lambda_{1}+c_{3} \Lambda_{1}^{2} .
$$

The first term is constrained in that $Q_{1}$ is monic, and due to units only three $u$-independent terms remain. The decoupling (B4) to $N_{f}=0$ implies that $c_{1}=-\frac{4}{3}$. From the massless limit we find $c_{3}=0$. On $N_{f}=1$ there are three $\mathrm{AD}$ theories with mass $m_{1}=\frac{3}{4} \omega_{3}$ (where $\omega_{3}^{3}=1$ ), for which $Q_{1}$ is known. This gives three additional equations for $c_{2}$, which all imply $c_{2}=0$. As anticipated, we find $Q_{1}=P_{1}^{\mathrm{M}}$.

For $N_{f}=2,3$ there are more terms in a general ansatz for $Q_{N_{f}}$, however many are fixed from (B4), the other above mentioned constraints and the limits to zero and AD mass. One can check that $Q_{2}=P_{2}^{\mathrm{M}}$ and $Q_{3}=P_{3}^{\mathrm{M}}$. This gives a proof of (3.13) that is independent to the one for (3.12).

\section{APPENDIX C: BETA FUNCTIONS OF MASSIVE THEORIES}

As discussed in Sec. VII, our work suggests to generalize the proposal of $[42,43]$ to conjecture that the beta functions of the massive theories (at least for $N_{f}=2,3$ ) are given by

$$
\beta_{N_{f}}=-\frac{\Delta}{P_{N_{f}}^{\mathrm{M}}} \frac{1}{u} \frac{d \tau}{d u}=\frac{4-N_{f}}{16 \pi i} \frac{1}{u}\left(\frac{d u}{d a}\right)^{2} .
$$

Note that the second formula immediately implies that the beta functions vanish at the $\mathrm{AD}$ points, since we know that $\frac{d u}{d a}\left(\tau_{\mathrm{AD}}\right)=0$ and $u\left(\tau_{\mathrm{AD}}\right)$ is finite. This is of course the expected behavior since these theories are superconformal. Note, however, that this is not the case for the proposal $\beta=-\frac{1}{u} \frac{d \tau}{d u}$ given in Refs. [31,102], which is related to the critique raised in Refs. [104,105]. We also have that, in the modular theories, $\frac{d u}{d a}$ is a weight -2 modular form of the corresponding monodromy group, and therefore we see that this also applies to $\beta_{N_{f}}$. We can make some further checks of this conjecture in the two cases $N_{f}=2$ with equal mass and $N_{f}=3$ with one nonzero mass where we have explicit expressions for all the relevant quantities. Similar analyses could be made for the theories where the mass has been tuned to the $\mathrm{AD}$ values, but we leave that for the interested reader.

Using the formulas of Sec. V we find that the proposed beta function of equal mass $N_{f}=2$ is given by

$\beta_{2}=\frac{1}{8 \pi i} \frac{1}{u}\left(\frac{d u}{d a}\right)^{2}=\frac{1}{\pi i} \frac{\vartheta_{2}^{4}+\vartheta_{3}^{4}+\sqrt{f_{2}}}{\vartheta_{4}^{8}+\vartheta_{2}^{4} \vartheta_{3}^{4}+\left(\vartheta_{2}^{4}+\vartheta_{3}^{4}\right) \sqrt{f_{2}}}$, with $f_{2}=\vartheta_{4}^{8}+16 \frac{m^{2}}{\Lambda_{2}^{2}} \vartheta_{2}^{4} \vartheta_{3}^{4}$. For $N_{f}=3$ with one mass the formulas of Sec. VI instead gives us

$\beta_{3}=\frac{1}{16 \pi i} \frac{1}{u}\left(\frac{d u}{d a}\right)^{2}=\frac{1}{2 \pi i} \frac{\vartheta_{3}^{4}+\vartheta_{4}^{4}+2 \sqrt{f_{3}}}{2 \vartheta_{3}^{4} \vartheta_{4}^{4}+\left(\vartheta_{3}^{4}+\vartheta_{4}^{4}\right) \sqrt{f_{3}}}$,

with $f_{3}=\vartheta_{3}^{4} \vartheta_{4}^{4}+64 \frac{m^{2}}{\Lambda_{3}^{2}} \vartheta_{2}^{8}$. Taking the massless limits in the above formulas of course gives back the expressions of Ref. [43] (here we are disregarding the shift in $u$ for $N_{f}=3$ ).

Let us study the behavior near the special points. For $\tau \rightarrow i \infty$ we immediately find

$$
\begin{aligned}
& \beta_{2}=-\frac{i}{\pi}+\mathcal{O}(q), \\
& \beta_{3}=-\frac{i}{2 \pi}+\mathcal{O}(q),
\end{aligned}
$$

which is the expected behavior [43]. We also need to study the behavior near the strong coupling singularities. For the two theories under study these singularities are given in Eqs. (5.1) and (6.2), and the transformations to get the relevant dual expressions for $\beta_{N_{f}}$ are given in the corresponding sections. For $N_{f}=2$ the behavior near each of these cusps is then

$$
\begin{aligned}
& \beta_{2,-}=\frac{2 i}{\pi} \frac{2 m+\Lambda_{2}}{8 m+\Lambda_{2}}+\mathcal{O}(q), \\
& \beta_{2, *}=-\frac{2 i}{\pi} \frac{\left(2 m-\Lambda_{2}\right)\left(2 m+\Lambda_{2}\right)}{8 m^{2}+\Lambda_{2}^{2}}+\mathcal{O}\left(q^{1 / 2}\right), \\
& \beta_{2,+}=\frac{2 i}{\pi} \frac{2 m-\Lambda_{2}}{8 m-\Lambda_{2}}+\mathcal{O}(q) .
\end{aligned}
$$

For $N_{f}=3$ we instead find

$$
\begin{aligned}
& \beta_{3,-}=\frac{i}{\pi} \frac{16 m+\Lambda_{3}}{16 m}+\mathcal{O}\left(q^{1 / 2}\right), \\
& \beta_{3, *}=-\frac{i}{2 \pi} \frac{\left(16 m-\Lambda_{3}\right)\left(16 m+\Lambda_{3}\right)}{256 m^{2}+\Lambda_{3}^{2}}+\mathcal{O}(q), \\
& \beta_{3,+}=\frac{i}{\pi} \frac{16 m-\Lambda_{3}}{16 m}+\mathcal{O}\left(q^{1 / 2}\right) .
\end{aligned}
$$

We can now make the following observations. We can explicitly see that the beta functions vanish at the AD points by recognizing that in $N_{f}=2$ equal mass we have AD points for $m= \pm \frac{\Lambda_{2}}{2}$, and tuning the mass to one of these values will merge either $u_{+}$or $u_{-}$with $u_{*}$, and we see that the corresponding contributions are exactly the ones that vanish for this specific value. Similarly, in $N_{f}=3$ with one mass the AD value is $m= \pm \frac{\Lambda_{3}}{16}$, and this will again merge either $u_{+}$or $u_{-}$with $u_{*}$, and the corresponding contributions exactly vanish. The beta functions also seem to blow 
up for specific values of the mass, these are exactly the values for which the corresponding singularity gets moved to $u=0$, for example if $m=0$ in $N_{f}=3 u_{+}$and $u_{-}$will meet at $u=0$. This behavior at $u=0$ is of course already noted in Ref. [43] and it is claimed that it corresponds to repulsive fixed points.
[1] C. Montonen and D. I. Olive, Magnetic monopoles as gauge particles?, Phys. Lett. B 72, 117 (1977).

[2] A. Sen, Strong-weak coupling duality in fourdimensional string theory, Int. J. Mod. Phys. A 09, 3707 (1994).

[3] N. Seiberg and E. Witten, Electric-magnetic duality, monopole condensation, and confinement in $N=2$ supersymmetric Yang-Mills theory, Nucl. Phys. B426, 19 (1994).

[4] C. Vafa and E. Witten, A strong coupling test of S duality, Nucl. Phys. B431, 3 (1994).

[5] E. P. Verlinde, Global aspects of electric - magnetic duality, Nucl. Phys. B455, 211 (1995).

[6] N. Seiberg and E. Witten, Monopoles, duality and chiral symmetry breaking in $N=2$ supersymmetric QCD, Nucl. Phys. B431, 484 (1994).

[7] A. Klemm, W. Lerche, S. Yankielowicz, and S. Theisen, Simple singularities and $N=2$ supersymmetric YangMills theory, Phys. Lett. B 344, 169 (1995).

[8] A. Klemm, W. Lerche, S. Yankielowicz, and S. Theisen, On the monodromies of $N=2$ supersymmetric YangMills theory, arXiv:hep-th/9412158.

[9] A. Klemm, W. Lerche, and S. Theisen, Nonperturbative effective actions of $N=2$ supersymmetric gauge theories, Int. J. Mod. Phys. A 11, 1929 (1996).

[10] U. H. Danielsson and B. Sundborg, The moduli space and monodromies of $N=2$ supersymmetric $S O(2 r+1)$ Yang-Mills theory, Phys. Lett. B 358, 273 (1995).

[11] G. W. Moore and E. Witten, Integration over the $u$ plane in Donaldson theory, Adv. Theor. Math. Phys. 1, 298 (1997).

[12] A. Losev, N. Nekrasov, and S. L. Shatashvili, Issues in topological gauge theory, Nucl. Phys. B534, 549 (1998).

[13] A. Malmendier and K. Ono, SO(3)-Donaldson invariants of $\mathbb{P}^{2}$ and mock theta functions, Geom. Topol. 16, 1767 (2012).

[14] M. Griffin, A. Malmendier, and K. Ono, SU(2)-Donaldson invariants of the complex projective plane, Forum mathematicum 27, 2003 (2015).

[15] A. Malmendier and K. Ono, Moonshine and Donaldson invariants of $\mathbb{P}^{2}$, Commun. Number Theory Phys. 6, 759 (2012).

[16] A. Malmendier, Donaldson invariants of $\mathbb{P}^{1} \times \mathbb{P}^{1}$ and mock theta functions, Commun. Num. Theor. Phys. 5, 203 (2011).

[17] G. Korpas and J. Manschot, Donaldson-Witten theory and indefinite theta functions, J. High Energy Phys. 11 (2017) 083.

[18] G. W. Moore and I. Nidaiev, The partition function of Argyres-Douglas theory on a four-manifold, arXiv:1711 .09257 .
[19] G. Korpas, Donaldson-Witten theory, surface operators and mock modular forms, arXiv:1810.07057.

[20] G. Korpas, J. Manschot, G. Moore, and I. Nidaiev, Renormalization and BRST symmetry in DonaldsonWitten theory, Ann. Henri Poincaré 20, 3229 (2019).

[21] G. Korpas, J. Manschot, G. W. Moore, and I. Nidaiev, Mocking the $u$-plane integral, arXiv:1910.13410.

[22] J. Manschot and G. W. Moore, Topological correlators of $S U(2), \mathcal{N}=2^{*}$ SYM on four-manifolds, arXiv:2104 .06492 .

[23] M. Marino and G. Moore, Integrating over the coulomb branch in $n=2$ gauge theory, Nucl. Phys. B, Proc. Suppl. 68, 336 (1998).

[24] M. Marino and G. W. Moore, Donaldson invariants for nonsimply connected manifolds, Commun. Math. Phys. 203, 249 (1999).

[25] S. Hyun, J. Park, and J.-S. Park, $N=2$ supersymmetric QCD and four manifolds: 1. The Donaldson and SeibergWitten invariants, arXiv:hep-th/9508162.

[26] A. Klemm, On the geometry behind $N=2$ supersymmetric effective actions in four-dimensions, arXiv:hep-th/ 9705131.

[27] E. D'Hoker and D. H. Phong, Lectures on supersymmetric Yang-Mills theory and integrable systems, in Theoretical Physics at the End of the Twentieth Century. Proceedings, Summer School, Banff, Canada, 1999 (1999), pp. 1-125.

[28] M. Matone, Koebe $1 / 4$ theorem and inequalities in $n=2$ supersymmetric QCD, Phys. Rev. D 53, 7354 (1996).

[29] W. Nahm, On the Seiberg-Witten approach to electricmagnetic duality, arXiv:hep-th/9608121.

[30] K. Ito and S.-K. Yang, Prepotentials in $N=2 \mathrm{SU}(2)$ supersymmetric Yang-Mills theory with massless hypermultiplets, Phys. Lett. B 366, 165 (1996).

[31] H. Kanno and S.-K. Yang, Donaldson-Witten functions of massless $N=2$ supersymmetric QCD, Nucl. Phys. B535, 512 (1998).

[32] M.-x. Huang and A. Klemm, Holomorphicity and modularity in Seiberg-Witten theories with matter, J. High Energy Phys. 07 (2010) 083.

[33] P. C. Argyres and M. R. Douglas, New phenomena in SU(3) supersymmetric gauge theory, Nucl. Phys. B448, 93 (1995).

[34] P. C. Argyres, M. R. Plesser, N. Seiberg, and E. Witten, New $N=2$ superconformal field theories in fourdimensions, Nucl. Phys. B461, 71 (1996).

[35] T. Eguchi, K. Hori, K. Ito, and S.-K. Yang, Study of $N=2$ superconformal field theories in four-dimensions, Nucl. Phys. B471, 430 (1996). 
[36] T. Eguchi and K. Hori, $N=2$ superconformal field theories in four-dimensions and A-D-E classification, arXiv:hep-th/9607125.

[37] J. Seo and K. Dasgupta, Argyres-Douglas loci, singularity structures and wall-crossings in pure $N=2$ gauge theories with classical gauge groups, J. High Energy Phys. 05 (2012) 072.

[38] D. Xie, General Argyres-Douglas theory, J. High Energy Phys. 01 (2013) 100.

[39] M. Matone, Instantons and recursion relations in $N=2$ SUSY gauge theory, Phys. Lett. B 357, 342 (1995).

[40] E. Witten, On S duality in Abelian gauge theory, Sel. Math. Sov. 1, 383 (1995).

[41] J. Manschot, G. W. Moore, and X. Zhang, Effective gravitational couplings of four-dimensional $\mathcal{N}=2$ supersymmetric gauge theories, J. High Energy Phys. 06 (2020) 150.

[42] B. P. Dolan, $N=2$ supersymmetric Yang-Mills and the quantum hall effect, Int. J. Mod. Phys. A 21, 4807 (2006).

[43] B. P. Dolan, Meromorphic scaling flow of $N=2$ supersymmetric SU(2) Yang-Mills with matter, Nucl. Phys. B737, 153 (2006).

[44] E. Witten, Solutions of four-dimensional field theories via M theory, Nucl. Phys. B500, 3 (1997).

[45] A. Klemm, W. Lerche, P. Mayr, C. Vafa, and N. P. Warner, Selfdual strings and $N=2$ supersymmetric field theory, Nucl. Phys. B477, 746 (1996).

[46] D. Gaiotto, G. W. Moore, and A. Neitzke, Wall-crossing, hitchin systems, and the WKB approximation, arXiv:0907 .3987 .

[47] A. Malmendier, The signature of the Seiberg-Witten surface, Surveys Diff. Geom. 15, 255 (2010).

[48] T. Shioda, On elliptic modular surfaces, J. Math. Soc. Jpn. 24, 20 (1972).

[49] M. Schütt and T. Shioda, Elliptic surfaces, in Mordell-Weil Lattices , Ergebnisse der Mathematik und ihrer Grenzgebiete. 3. Folge / A Series of Modern Surveys in Mathematics Vol. 70 (Springer, Singapore, 2009), 10.1007/978981-32-9301-4_5.

[50] R. S. Maier, On rationally parametrized modular equations, arXiv:math/0611041.

[51] R. Miranda, An overview of algebraic surfaces, Lect. Notes Pure and Appl. Math. 193, 157 (1997).

[52] T. Eguchi, Seiberg-Witten Theory and S-Duality (Springer Netherlands, Dordrecht, 1999), pp. 103-120.

[53] G. Shimura, Introduction to the Arithmetic Theory of Automorphic Functions Kanô Memorial Lectures (Princeton University Press, Princeton, NJ, 1971).

[54] A. Khovanskii, Galois Theory, Coverings, and Riemann Surfaces (Springer, Berlin, Heidelberg, 2013).

[55] C. Y. Park, Ramification points of Seiberg-Witten curves, J. High Energy Phys. 07 (2011) 068.

[56] A. Bourget and J. Troost, Permutations of massive vacua, J. High Energy Phys. 05 (2017) 042.

[57] F. Ferrari, On the geometry of super Yang-Mills theories: Phases and irreducible polynomials, J. High Energy Phys. 01 (2009) 026.

[58] F. Ferrari, Galois symmetries in super Yang-Mills theories, J. High Energy Phys. 03 (2009) 128.
[59] Y.-H. He and J. Read, Hecke groups, dessins d'Enfants and the archimedean solids, Front. Phys. 3, 91 (2015).

[60] S. K. Ashok, F. Cachazo, and E. Dell'Aquila, Children's drawings from Seiberg-Witten curves, Commun. Num. Theor. Phys. 1, 237 (2007).

[61] S. K. Ashok, F. Cachazo, and E. Dell'Aquila, Strebel differentials with integral lengths and Argyres-Douglas singularities, arXiv:hep-th/0610080.

[62] Y.-H. He and J. McKay, $N=2$ gauge theories: Congruence subgroups, coset graphs and modular surfaces, J. Math. Phys. (N.Y.) 54, 012301 (2013).

[63] K. Kodaira, On compact analytic surfaces: II, Ann. Math. 77, 563 (1963).

[64] K. Kodaira, On compact analytic surfaces: III, Ann. Math. 78, 1 (1963).

[65] J. Sonnenschein, S. Theisen, and S. Yankielowicz, On the relation between the holomorphic prepotential and the quantum moduli in susy gauge theories, Phys. Lett. B 367, 145 (1996).

[66] T. Eguchi and S.-K. Yang, Prepotentials of $N=2$ supersymmetric gauge theories and soliton equations, Mod. Phys. Lett. A 11, 131 (1996).

[67] R. Flume, F. Fucito, J. F. Morales, and R. Poghossian, Matone's relation in the presence of gravitational couplings, J. High Energy Phys. 04 (2004) 008.

[68] F. Diamond and J. Shurman, A First Course in Modular Forms, 1st ed., Graduate Texts in Mathematics Vol. 228 (Springer-Verlag, New York, 2005).

[69] A. Brandhuber and S. Stieberger, Periods, coupling constants and modular functions in $N=2 \mathrm{SU}(2) \mathrm{SYM}$ with massive matter, Int. J. Mod. Phys. A 13, 1329 (1998).

[70] J. Aspman, E. Furrer, and J. Manschot, Four flavours, triality and bimodular forms, arXiv:2110.11969.

[71] T. Eguchi and H. Kanno, Five-dimensional gauge theories and local mirror symmetry, Nucl. Phys. B586, 331 (2000).

[72] Y. Ohta, Prepotential of $N=2$ SU(2) Yang-Mills gauge theory coupled with a massive matter multiplet, J. Math. Phys. (N.Y.) 37, 6074 (1996).

[73] Y. Ohta, Prepotentials of $N=2$ SU(2) Yang-Mills theories coupled with massive matter multiplets, J. Math. Phys. (N.Y.) 38, 682 (1997).

[74] M.-x. Huang, A.-K. Kashani-Poor, and A. Klemm, The $\Omega$ deformed B-model for rigid $\mathcal{N}=2$ theories, Ann. Henri Poincaré 14, 425 (2013).

[75] L. Alvarez-Gaume, M. Marino, and F. Zamora, Softly broken $N=2$ QCD with massive quark hypermultiplets. 2., Int. J. Mod. Phys. A 13, 1847 (1998).

[76] L. Alvarez-Gaume, M. Marino, and F. Zamora, Softly broken $N=2$ QCD with massive quark hypermultiplets. 1., Int. J. Mod. Phys. A 13, 403 (1998).

[77] B. P. Dolan, Modular symmetry and fractional charges in $N=2$ supersymmetric Yang-Mills and the quantum hall effect, SIGMA 3, 010 (2007).

[78] J. Aspman, E. Furrer, and J. Manschot, Elliptic loci of SU(3) vacua, Ann. Henri Poincaré 22, 2775 (2021).

[79] A. Klemm, J. Manschot, and T. Wotschke, Quantum geometry of elliptic Calabi-Yau manifolds, arXiv:1205.1795.

[80] J. H. Conway and S. P. Norton, Monstrous moonshine, Bull. London Math. Soc. 11, 308 (1979). 
[81] D. Schultz, Notes on modular forms, https://faculty.math .illinois.edu/ schult25/ModFormNotes.pdf.

[82] P. Argyres, M. Lotito, Y. Lü, and M. Martone, Geometric constraints on the space of $\mathcal{N}=2$ SCFTs. Part I: Physical constraints on relevant deformations, J. High Energy Phys. 02 (2018) 001.

[83] T. Masuda and H. Suzuki, Periods and prepotential of $N=2$ SU(2) supersymmetric Yang-Mills theory with massive hypermultiplets, Int. J. Mod. Phys. A 12, 3413 (1997).

[84] A. Bilal and F. Ferrari, The BPS spectra and superconformal points in massive $N=2$ supersymmetric QCD, Nucl. Phys. B516, 175 (1998).

[85] T. Gamelin, Complex Analysis, Undergraduate Texts in Mathematics (Springer, New York, 2003).

[86] D. Alexander, C. Cummins, J. McKay, and C. Simons, Completely replicable functions, in Groups, Combinatorics, and Geometry, Durham Symposium, 1990, London Mathematical Society Lecture Note Series Vol. 165 (Cambridge University Press, Cambridge, England, 1992).

[87] D. Ford, J. McKay, and S. Norton, More on replicable functions, Communications in algebra 22, 5175 (1994).

[88] C. R. Ferenbaugh, The genus-zero problem for $n \mid h$-type groups, Duke Math. J. 72, 31 (1993).

[89] J. Juan-Zacarías and A. Verjovsky, Some remarks on equilateral triangulations of surfaces and Belyi functions, arXiv:2002.04660.

[90] G. Almkvist and J. Guillera, Ramanujan-Sato-like series, in Number Theory and Related Fields, edited by J. M. Borwein, I. Shparlinski, and W. Zudilin (Springer, New York, 2013), pp. 55-74.

[91] H. Chan, S. Chan, and Z.-G. Liu, Domb's numbers and Ramanujan-Sato type series for $1 / \pi$, Adv. Math. 186, 396 (2004).

[92] H. H. Chan, Y. Tanigawa, Y. Yang, and W. Zudilin, New analogues of clausens identities arising from the theory of modular forms, Adv. Math. 228, 1294 (2011).

[93] H. Chan and S. Cooper, Rational analogues of Ramanujan's series for $1 / \pi$, Math. Proc. Cambridge Philos. Soc. 153, 361 (2012).

[94] V. Tatitscheff, Y.-H. He, and J. McKay, Cusps, congruence groups and monstrous dessins, arXiv:1812.11752.

[95] Y.-H. He and M. van Loon, Gauge theories, tessellations \& Riemann surfaces, J. High Energy Phys. 06 (2014) 053.

[96] D. Gaiotto, $N=2$ dualities, J. High Energy Phys. 08 (2012) 034.

[97] N. Hajouji and P.-K. Oehlmann, Modular curves and Mordell-Weil torsion in F-theory, J. High Energy Phys. 04 (2020) 103.

[98] N. Seiberg, Five-dimensional SUSY field theories, nontrivial fixed points and string dynamics, Phys. Lett. B 388, 753 (1996).

[99] E. Witten, Monopoles and four manifolds, Math. Res. Lett. 1, 769 (1994).

[100] J. G. Russo, $\mathcal{N}=2$ gauge theories and quantum phases, J. High Energy Phys. 12 (2014) 169.
[101] J. G. Russo, Properties of the partition function of $\mathcal{N}=2$ supersymmetric QCD with massive matter, J. High Energy Phys. 07 (2019) 125.

[102] J. A. Minahan and D. Nemeschansky, $N=2$ superYangMills and subgroups of SL(2,Z), Nucl. Phys. B468, 72 (1996).

[103] A. Ritz, On the beta function in $N=2$ supersymmetric Yang-Mills theory, Phys. Lett. B 434, 54 (1998).

[104] G. Carlino, K. Konishi, N. Maggiore, and N. Magnoli, On the beta function in supersymmetric gauge theories, Phys. Lett. B 455, 171 (1999).

[105] K. Konishi, Renormalization group and dynamics of supersymmetric gauge theories, Int. J. Mod. Phys. A 16, 1861 (2001).

[106] C. Closset and H. Magureanu, The $U$-plane of rank-one $4 \mathrm{~d}$ $\mathcal{N}=2$ KK theories, arXiv:2107.03509.

[107] G. H. J. H. Bruinier, G. van der Geer, and D. Zagier, The 1-2-3 of Modular Forms (Springer-Verlag, Berlin, Heidelberg, 2008).

[108] K. Ono, The Web of Modularity: Arithmetic of the Coefficients of Modular Forms and q-Series, Vol. 102 American Mathematical Society, CBMS Regional Conference Series in Mathematics (American Mathematical Society, Providence, Rhode Island, 2004).

[109] D. Zagier, Introduction to Modular Forms; From Number Theory to Physics (Springer, Berlin, 1992), pp. 238-291.

[110] N. Koblitz, Introduction to Elliptic Curves and Modular Forms, Graduate Texts in Mathematics (Springer, New York, 1993).

[111] K. H. B. Gordon, Multiplicative Properties of Eta-Products, Vol. 143 of Contemporary Mathematics (American Mathematical Society, Providence, 1993).

[112] A. Sebbar, Classification of torsion-free genus zero congruence groups, Proc. Am. Math. Soc. 129, 2517 (2001).

[113] S. Alexandre Vidal, Sur la classification et le denombrement des sous-groupes du groupe modulaire et de leurs classes de conjugaison, arXiv:math/0702223.

[114] K. Wohlfahrt, An extension of f. klein's level concept, Ill. J. Math. 8, 529 (1964).

[115] R. S. Maier, Nonlinear differential equations satisfied by certain classical modular forms, Manuscr. Math. 134, 1 (2011).

[116] T. Weigand, F-theory, Proc. Sci. TASI2017 (2018) 016 [arXiv:1806.01854].

[117] M. Martone, Towards the classification of rank-r $\mathcal{N}=2$ SCFTs. Part I: Twisted partition function and central charge formulae, J. High Energy Phys. 12 (2020) 021.

[118] P. C. Argyres and M. Martone, Towards a classification of rank $r \mathcal{N}=2$ SCFTs Part II: Special Kahler stratification of the Coulomb branch, J. High Energy Phys. 12 (2020) 022.

[119] Y. Tachikawa, N=2 Supersymmetric Dynamics for Pedestrians, Lecture Notes in Physics Vol. 890 (Springer, Cham, 2013), 10.1007/978-3-319-08822-8. 